\title{
Measuring nickel masses in Type Ia supernovae using cobalt emission in nebular phase spectra
}

Michael J. Childress ${ }^{1,2 \star}$, D. John Hillier ${ }^{3}$, Ivo Seitenzahl ${ }^{1,2}$, Mark Sullivan ${ }^{4}$, Kate Maguire ${ }^{5}$, Stefan Taubenberger ${ }^{5}$, Richard Scalzo $^{1}$, Ashley Ruiter ${ }^{1,2}$, Nadejda Blagorodnova ${ }^{7}$, Yssavo Camacho ${ }^{8,9}$, Jayden Castillo ${ }^{1}$, Nancy Elias-Rosa ${ }^{10}$, Morgan Fraser ${ }^{7}$, Avishay Gal-Yam ${ }^{11}$, Melissa Graham ${ }^{12}$, D. Andrew Howell ${ }^{13,14}$, Cosimo Inserra ${ }^{15}$, Saurabh W. Jha ${ }^{9}$, Sahana Kumar ${ }^{12}$, Paolo A. Mazzali ${ }^{16,17}$, Curtis McCully ${ }^{13,14}$, Antonia Morales-Garoffolo ${ }^{18}$, Viraj Pandya ${ }^{19,9}$, Joe Polshaw ${ }^{15}$, Brian Schmidt ${ }^{1}$, Stephen Smartt ${ }^{15}$, Ken W. Smith ${ }^{15}$, Jesper Sollerman ${ }^{20}$, Jason Spyromilio ${ }^{5}$, Brad Tucker $^{1,2}$, Stefano Valenti ${ }^{13,14}$, Nicholas Walton ${ }^{7}$, Christian Wolf ${ }^{1}$, Ofer Yaron $^{11}$, D. R. Young ${ }^{15}$, Fang Yuan ${ }^{1,2}$, Bonnie Zhang ${ }^{1,2}$

\author{
${ }^{1}$ Research School of Astronomy and Astrophysics, Australian National University, Canberra, ACT 2611, Australia. \\ ${ }^{2}$ ARC Centre of Excellence for All-sky Astrophysics (CAASTRO). \\ ${ }^{3}$ Department of Physics and Astronomy \& Pittsburgh Particle Physics, Astrophysics, and Cosmology Center (PITT PACC), University of Pittsburgh, 3941 \\ O'Hara Street, Pittsburgh, PA 15260, USA. \\ ${ }^{4}$ School of Physics and Astronomy, University of Southampton, Southampton, SO17 1BJ, UK. \\ ${ }^{5}$ European Organisation for Astronomical Research in the Southern Hemisphere (ESO), Karl-Schwarzschild-Str. 2, 85748 Garching b. München, Germany. \\ ${ }^{7}$ Institute of Astronomy, University of Cambridge, Madingley Rd., Cambridge, CB3 OHA, UK. \\ ${ }^{8}$ Department of Physics, Lehigh University, 16 Memorial Drive East, Bethlehem, Pennsylvania 18015, USA. \\ ${ }^{9}$ Department of Physics and Astronomy, Rutgers, the State University of New Jersey, 136 Frelinghuysen Road, Piscataway, NJ 08854, USA. \\ ${ }^{10}$ INAF - Osservatorio Astronomico di Padova, vicolo dell'Osservatorio 5, 35122 Padova, Italy. \\ ${ }^{11}$ Department of Particle Physics and Astrophysics, The Weizmann Institute of Science, Rehovot 76100, Israel. \\ ${ }^{12}$ Department of Astronomy, University of California, Berkeley, CA 94720-3411, USA. \\ ${ }^{13}$ Department of Physics, University of California, Broida Hall, Mail Code 9530, Santa Barbara, CA 93106-9530, USA. \\ ${ }^{14}$ Las Cumbres Observatory Global Telescope Network, 6740 Cortona Dr., Suite 102, Goleta, CA 93117, USA. \\ ${ }^{15}$ Astrophysics Research Centre, School of Mathematics and Physics, Queen's University Belfast, Belfast BT7 1NN, UK. \\ ${ }^{16}$ Astrophysics Research Institute, Liverpool John Moores University, Egerton Wharf, Birkenhead, CH41 1LD, UK. \\ ${ }^{17}$ Max-Planck-Institut für Astrophysik, Karl-Schwarzschild str. 1, 85748 Garching, Germany. \\ 18 Institut de Ciències de l'Espai (CSIC-IEEC), Campus UAB, Camí de Can Magrans S/N, 08193 Cerdanyola, Spain. \\ ${ }^{19}$ Department of Astrophysical Sciences, Princeton University, Princeton, NJ 08544, USA. \\ ${ }^{20}$ The Oskar Klein Centre, Department of Astronomy, AlbaNova, Stockholm University, 10691 Stockholm, Sweden.
}

\begin{abstract}
The light curves of Type Ia supernovae (SNe Ia) are powered by the radioactive decay of ${ }^{56} \mathrm{Ni}$ to ${ }^{56} \mathrm{Co}$ at early times, and the decay of ${ }^{56} \mathrm{Co}$ to ${ }^{56} \mathrm{Fe}$ from $\sim 60$ days after explosion. We examine the evolution of the [Co III] $\lambda 5893$ emission complex during the nebular phase for SNe Ia with multiple nebular spectra and show that the line flux follows the square of the mass of ${ }^{56} \mathrm{Co}$ as a function of time. This result indicates both efficient local energy deposition from positrons produced in ${ }^{56} \mathrm{Co}$ decay, and long-term stability of the ionization state of the nebula. We compile 77 nebular spectra of $25 \mathrm{SN}$ Ia from the literature and present 17 new nebular spectra of 7 SNe Ia, including SN 2014J. From these we measure the flux in the [Co III] $\lambda 5893$ line and remove its well-behaved time dependence to infer the initial mass of ${ }^{56} \mathrm{Ni}\left(M_{N i}\right)$ produced in the explosion. We then examine ${ }^{56} \mathrm{Ni}$ yields for different $\mathrm{SN}$ Ia ejected masses $\left(M_{e j}\right.$ - calculated using the relation between light curve width and ejected mass) and find the ${ }^{56} \mathrm{Ni}$ masses of $\mathrm{SNe}$ Ia fall into two regimes: for narrow light curves (low stretch $s \sim 0.7-0.9), M_{N i}$ is clustered near $M_{N i} \approx 0.4 M_{\odot}$ and shows a shallow increase as $M_{e j}$ increases from $\sim 1-1.4 M_{\odot}$; at high stretch, $M_{e j}$ clusters at the Chandrasekhar mass $\left(1.4 M_{\odot}\right)$ while $M_{N i}$ spans a broad range from $0.6-1.2 M_{\odot}$. This could constitute evidence for two distinct SN Ia explosion mechanisms.
\end{abstract}

Key words: supernovae: general 


\section{INTRODUCTION}

Type Ia supernovae ( $\mathrm{SNe}$ Ia) were instrumental to the discovery of the accelerating expansion of the Universe (Riess et al. 1998; Perlmutter et al. 1999) and remain key tools for characterizing the precise cosmology of the Universe (Kessler et al. 2009; Sullivan et al. 2011; Rest et al. 2014; Betoule et al. 2014). Their cosmological utility is facilitated both by their intrinsic brightness $\left(M_{B} \sim-19\right.$ at peak) and the relative uniformity of their peak brightnesses. More importantly, their luminosity diversity is tightly correlated with the width of the optical light curve (Phillips 1993). The physical origin of this width-luminosity relation (WLR) has long been a subject of debate and is intimately tied to the progenitor system of SNe Ia and the physical mechanism that triggers the explosion.

$\mathrm{SNe}$ Ia are widely believed to result from the thermonuclear disruption of a carbon-oxygen (CO) white dwarf (Hoyle \& Fowler 1960), which has recently been supported observationally for the very nearby SN 2011fe (Bloom et al. 2012; Nugent et al. 2011). The CO-rich material in a white dwarf (WD) is supported against gravitational collapse by electron degeneracy pressure. A stable isolated WD lacks the internal pressure and temperature necessary to fuse CO to heavier elements (but see Chiosi et al. 2015). In $\mathrm{SNe}$ Ia, this balance is upset by interaction with some binary companion, which triggers runaway nuclear fusion of the $\mathrm{CO}$ material to heavier elements, particularly iron group elements (IGEs) dominated by radioactive ${ }^{56} \mathrm{Ni}$. The energy from fusion unbinds the star and ejects material at $\sim 10^{4} \mathrm{~km} \mathrm{~s}^{-1}$. As the ejecta expand the decay of ${ }^{56} \mathrm{Ni}$ to ${ }^{56} \mathrm{Co}$ (with half-life of $t_{1 / 2}=6.08$ days) releases energy into the ejecta which powers the optical lightcurve of the SN for the first few weeks after explosion (Colgate \& McKee 1969), including the luminous peak. At later epochs $(t \gtrsim 60$ days past peak brightness), the SN Ia lightcurve is powered by ${ }^{56} \mathrm{Co}$ decay to ${ }^{56} \mathrm{Fe}$ (with half-life of $t_{1 / 2}=77.2$ days). Thus understanding the origin of the trigger mechanism and the amount of ${ }^{56} \mathrm{Ni}$ produced in the explosion would reveal the critical elements that make SNe Ia such excellent cosmological tools.

The nature of the CO-WD binary companion is directly responsible for the event that triggers the SN Ia explosion. One possible scenario is the single degenerate (SD; Whelan \& Iben 1973; Nomoto 1982) scenario in which a CO-WD steadily accretes from a non-degenerate (main sequence or giant-like) companion until the central density of the WD exceeds the critical density for carbon ignition (e.g., Gasques et al. 2005) as the mass approaches the Chandrasekhar mass $\left(M_{W D} \approx 1.4 M_{\odot}\right)$. In this scenario, the WLR has been proposed to arise from stochastic variations in the time at which the nuclear burning front within the exploding WD transitions from sub-sonic to super-sonic - the so-called deflagration to detonation transition (DDT; e.g., Blinnikov \& Khokhlov 1986; Röpke \& Niemeyer 2007; Kasen \& Woosley 2007; Kasen et al. 2009; Sim et al. 2013). Variations in the time of the DDT result in different amounts of ${ }^{56} \mathrm{Ni}$ being produced, yielding different peak magnitudes and light curve widths for SNe Ia (though Sim et al. 2013, do not recover the observed WLR).

The other popular scenario for SN Ia progenitor systems is the double degenerate (DD; Tutukov \& Iungelson 1976; Tutukov \& Yungelson 1979; Iben \& Tutukov 1984; Webbink 1984) scenario in which two WDs in a close binary merge after orbital decay due to gravitational radiation. Some recent simulation results have shown that a violent merger of the two WDs produces "hot spots" which exceed the critical temperature and density (Seitenzahl et al. 2009a) needed to ignite CO fusion
(Guillochon et al. 2010; Lorén-Aguilar et al. 2010; Pakmor et al. 2010, 2013; Moll et al. 2014; Raskin et al. 2014). This scenario is inherently not tied to $M_{C h}$, but instead could produce explosions with varying luminosities and light curve widths simply due to the variation in mass of the progenitor system (Ruiter et al.2013). Generally for the DD scenario, the WD undergoes a complete detonation and the amount of ${ }^{56} \mathrm{Ni}$ produced depends on the mass of the progenitor (Fink et al. 2010; Sim et al. 2010).

Finally, it is important to also consider the double detonation (DDet) mechanism for triggering the WD explosion. In this scenario, helium-rich material accreted onto the surface of the white dwarf (either from a He-rich main sequence or giant star or HeWD) could ignite and send a shockwave into the core of the star. This shock wave then triggers a second detonation near the WD core which initiates the thermonuclear runaway process (Livne 1990; Iben \& Tutukov 1991; Woosley \& Weaver 1994; Fink et al. 2010; Woosley \& Kasen 2011). This mechanism could arise from SD or DD systems, and is not tied to $M_{C h}$. Additionally, this mechanism may offer a favorable explanation for the presence of high-velocity features in early SN Ia spectra (Mazzali et al. 2005; Maguire et al. 2012; Childress et al. 2013c; Marion et al. 2013; Childress et al. 2014a; Maguire et al. 2014; Pan et al. 2015a; Silverman et al. 2015).

While much of the debate about SN Ia progenitors in the previous decade revolved around which single scenario was responsible for $\mathrm{SNe} \mathrm{Ia}$, recent results have pointed toward multiple progenitor channels being realized in nature. SN Ia rates studies yielded evidence for both short- and longlived progenitors (Mannucci et al. 2005; Scannapieco \& Bildsten 2005; Sullivan et al. 2006; Mannucci et al. 2006; Aubourg et al. 2008). The lack of a detected companion star to the progenitor of SN 2011fe (Li et al. 2011) and in SN Ia remnants (e.g. Schaefer \& Pagnotta 2012; Kerzendorf et al. 2012, 2013, 2014a) present individual cases where the DD scenario seems necessary, while strong emission from circum-stellar material in some nearby SNe Ia (Hamuy et al. 2003; Aldering et al. 2006; Dilday et al. 2012; Silverman et al. 2013c b) seems to indicate clear cases of the SD scenario.

For peculiar white dwarf supernovae, like the Type-Iax SN 2012Z, a luminous progenitor system has been detected and interpreted as the donor star (McCully et al. 2014a). Similarly, shock interaction of $\mathrm{SN}$ ejecta with a (non-degenerate) companion star has been detected in the early light curve of another peculiar, lowvelocity white dwarf SN (Cao et al. 2015). However such shock interaction is distinctly absent for several other SNe Ia observed continuously through the epoch of first light with the Kepler satellite (Olling et al. 2015). Additionally, a general dichotomy in the spectroscopic properties of SNe Ia appears evident (Maguire et al. 2014). Thus numerous lines of evidence now point to multiple SN Ia progenitor channels being active.

Variations in progenitor masses between different explosion mechanisms will manifest as diversity in the bolometric light curves of SNe Ia (Arnett 1982; Jeffery 1999; Stritzinger et al. 2006a; Röpke et al. 2012). Recently, Scalzo et al. (2014a) demonstrated that the ejected mass - hence the progenitor mass - of a SN Ia could be recovered to $10-15 \%$ precision, as tested on bolometric light curves derived from radiative transfer modelling of SN Ia explosion models with known input progenitor mass. Applying the same modelling technique to real data, Scalzo et al. (2014a) found evidence that the ejected mass varies in the range 0.9-1.4 $M_{\odot}$ among spectroscopically normal (Branch et al. 1993) $\mathrm{SNe}$ Ia and that the ejected mass also correlates strongly with the 
light curve width parameter used to standardize SN Ia distances in cosmology. The correlation between ejected mass and light curve width was exploited by Scalzo et al. (2014b) to measure the SN Ia ejected mass distribution: they found that $25-50 \%$ of all normal SNe Ia eject sub-Chandrasekhar masses, with most of the rest being consistent with Chandrasekhar-mass events (this is consistent with constraints from Galactic chemical evolution based on $\mathrm{Mn} / \mathrm{Fe}$ in the solar neighborhood - Seitenzahl et al. 2013a). SuperChandrasekhar-mass SNe Ia were found to be very rare, at most a few percent of all SNe Ia, consistent with previous measurements of the relative rate (Scalzo et al. 2012).

The diversity in ejected mass suggests a corresponding diversity in explosion mechanisms among normal SNe Ia. Further information about the explosion mechanism may also be encoded in the peak absolute magnitude distribution (Ruiter et al. 2013; Piro et al. 2014), the diversity in early SN Ia light curves (Dessart et al. 2014c), or in the relation between ${ }^{56} \mathrm{Ni}$ and ejected mass (Sim et al. 2010; Ruiter et al. 2013; Scalzo et al. 2014a). The ${ }^{56} \mathrm{Ni}$ mass is most commonly inferred from the peak absolute magnitude of the supernova (Arnett 1982), although with some modeldependent systematic errors (Branch 1992; Hoeflich \& Khokhlov 1996; Howell et al. 2009). The ${ }^{56} \mathrm{Ni}$ mass can also be inferred from detailed modelling of photospheric phase spectral times series (Stehle et al. 2005; Mazzali et al. 2008; Tanaka et al. 2011; Sasdelli et al. 2014; (Blondin et al.2015). Reliable alternative methods for measuring ${ }^{56} \mathrm{Ni}$ masses, with different model-dependent systematics, can thus in principle help to shed light on the explosion mechanisms and progenitor properties of SNe Ia.

In this work, we show that the amount of ${ }^{56} \mathrm{Ni}$ produced in the SN Ia explosion can be measured directly from signatures of its decay product ${ }^{56} \mathrm{Co}$ in nebular phase spectra of SNe Ia. Specifically, we employ the flux of the [Co III] $\lambda 5893$ line in spectra of SNe Ia in the nebular phase ( $t \geq 150$ days past maximum brightness) as a diagnostic of the mass of ${ }^{56} \mathrm{Co}$ at a given epoch. Kuchner et al. (1994) showed that the ratio of the flux of this line to the Fe III line at $\lambda 4700 \AA$ as a function of SN phase followed the expected temporal evolution of the $\mathrm{Co} / \mathrm{Fe}$ mass ratio, which they used as evidence for the presence of ${ }^{56} \mathrm{Ni}$ generated in the $\mathrm{SN}$ explosion. More recently the presence of ${ }^{56} \mathrm{Ni}$ has been directly confirmed through $\gamma$-ray line emission from ${ }^{56} \mathrm{Ni}$ (Diehl et al. 2014) and ${ }^{56} \mathrm{Co}$ Churazov et al. 2014) lines observed by the INTEGRAL satellite for the very nearby SN 2014J.

Previous studies of SN Ia nebular spectra have collected a modest sample of spectra (a few dozen) from which important scientific results were derived. Mazzali et al. (1998) found a strong correlation between the width of nebular emission lines (specifically the Fe III 4700 feature) with the SN light curve stretch, constituting evidence for greater ${ }^{56} \mathrm{Ni}$ production in more luminous slow-declining SNe Ia. This result was combined with detailed modelling of nebular spectra (especially the $7380 \AA$ nebular line presumed to arise from stable ${ }^{58} \mathrm{Ni}$ ) to infer a common explosion mechanism for SNe Ia (Mazzali et al. 2007). Nebular spectra have also been employed to place upper limits on hydrogen in the vicinity of normal SNe Ia (Leonard 2007; Shappee et al. 2013; Silverman et al. 2013a; Lundqvist et al. 2015). The lack of hydrogen in normal SNe Ia is in contrast to the strong hydrogen lines found in late phase spectra of SNe Ia which exhibited strong interaction during the photospheric phase (Silverman et al. 2013b). Velocity shifts in the purported Ni $7380 \AA$ nebular line were used to infer asymmetry in the inner core of SNe Ia (Maeda et al. $2010 \mathrm{a}$ b), which was also found to correlate with the optical colour and Si $6355 \AA$ velocity gradient during the photospheric phase
(Maeda et al. 2011). These line velocity shifts were found to also correlate with photospheric phase spectropolarimetry (Maund et al. 2010), indicating a general correlated asymmetric geometry for SNe Ia. These early results have generally been supported with greater statistics afforded by new large data sets such as the CfA sample (Blondin et al. 2012) and BSNIP (Silverman et al. 2013a).

Until recently, the nebular line at $5890 \AA$ was not frequently emphasized as a diagnostic of ${ }^{56} \mathrm{Co}$ due to its presumed association with emission from sodium (Kuchner et al. 1994; McClelland et al. 2013, are noteworthy exceptions). However Dessart et al. (2014b) showed definitively that this line arises primarily from cobalt for the majority of SNe Ia. We exploit this result to use the [Co III] $\lambda 5893$ line as a diagnostic of ${ }^{56} \mathrm{Ni}$ from a large sample of nebular SN Ia spectra compiled from both new observations and from the literature. Equipped with a sample of 77 spectra of $25 \mathrm{SNe}$ Ia from the literature and 17 new spectra of $7 \mathrm{SNe}$ Ia, we calculate the $a b$ solute flux of the nebular [Co III] $\lambda 5893$ line by scaling the spectra to flux-calibrated photometry measurements. With these calibrated fluxes we show that the temporal evolution of the absolute [Co III] $\lambda 5893$ line flux is highly consistent for SNe Ia with multiple nebular spectra. We exploit this result to place measurements from disparate epochs on a common scale. This allows us to meaningfully compare the line fluxes in order to determine the relative amount of ${ }^{56} \mathrm{Ni}$ produced by each $\mathrm{SN}$ Ia in our sample.

In Section 2 we present our compilation of literature SN Ia spectra and the new nebular SN Ia data released here. Section 3 presents our method for measuring the [Co III] $\lambda 5893$ flux from the spectra and scaling the spectra with the SN Ia photometry. We examine the temporal evolution of the [Co III] $\lambda 5893$ for $\mathrm{SNe}$ Ia with numerous nebular observations in Section 4 We then infer ${ }^{56} \mathrm{Ni}$ masses for our SN Ia sample in Section 5, and discuss the implications and limitations of our results in Section 6 Finally we conclude in Section 7

\section{SN Ia NEBULAR SPECTROSCOPY DATA}

The analysis in this work relies on a compilation of SN Ia nebular spectra from the literature as well as new observations. The full sample of literature and new late phase spectra are presented in Table 1. 


\section{Childress et al.}

Table 1. New and Literature Late Phase SN Ia Spectra

\begin{tabular}{|c|c|c|c|}
\hline $\mathrm{SN}$ & $\begin{array}{c}\text { Phase } t^{a} \\
\text { (days) }\end{array}$ & Obs. Date ${ }^{b}$ & Spec. Ref. ${ }^{c}$ \\
\hline \multirow[t]{6}{*}{ SN 1990N } & 160 & 19901217 & BSNIP \\
\hline & 186 & 19910112 & Gómez \& López (1998) \\
\hline & 227 & 19910222 & Gómez \& López (1998) \\
\hline & 255 & 19910322 & Gómez \& López (1998) \\
\hline & 280 & 19910416 & Gómez \& López (1998) \\
\hline & 333 & 19910608 & Gómez \& López (1998) \\
\hline \multirow[t]{5}{*}{ SN 1991T } & 113 & 19910819 & BSNIP \\
\hline & 186 & 19911031 & BSNIP \\
\hline & 258 & 19920111 & Gómez \& López (1998) \\
\hline & 320 & 19920313 & BSNIP \\
\hline & 349 & 19920411 & BSNIP \\
\hline \multirow[t]{2}{*}{ SN 1994ae } & 144 & 19950422 & BSNIP \\
\hline & 153 & 19950501 & $\mathrm{CfA}$ \\
\hline \multirow[t]{2}{*}{ SN 1995D } & 277 & 19951124 & CfA \\
\hline & 285 & 19951202 & CfA \\
\hline \multirow[t]{3}{*}{ SN 1998aq } & 211 & 19981124 & Branch et al. (2003) \\
\hline & 231 & 19981214 & Branch et al. (2003) \\
\hline & 241 & 19981224 & Branch et al. (2003) \\
\hline \multirow[t]{9}{*}{ SN 1998bu } & 179 & 19981114 & $\mathrm{CfA}$ \\
\hline & 190 & 19981125 & CfA \\
\hline & 208 & 19981213 & CfA \\
\hline & 217 & 19981222 & $\mathrm{CfA}$ \\
\hline & 236 & 19990110 & BSNIP \\
\hline & 243 & 19990117 & CfA \\
\hline & 280 & 19990223 & BSNIP \\
\hline & 329 & 19990413 & Cappellaro et al. (2001) \\
\hline & 340 & 19990424 & BSNIP \\
\hline \multirow[t]{2}{*}{ SN 1999aa } & 256 & 19991109 & BSNIP \\
\hline & 282 & 19991205 & BSNIP \\
\hline SN 2002cs & 174 & 20021106 & BSNIP \\
\hline \multirow{2}{*}{ SN 2002dj } & 222 & 20030201 & Pignata et al. (2008) \\
\hline & 275 & 20030326 & Pignata et al. (2008) \\
\hline SN 2002er & 216 & 20030410 & Kotak et al. (2005) \\
\hline SN 2002fk & 150 & 20030227 & BSNIP \\
\hline \multirow[t]{8}{*}{ SN 2003du } & 109 & 20030823 & Stanishev et al. (2007) \\
\hline & 138 & 20030921 & Anupama et al. (2005) \\
\hline & 139 & 20030922 & Anupama et al. (2005) \\
\hline & 142 & 20030925 & Stanishev et al. (2007) \\
\hline & 209 & 20031201 & Stanishev et al. (2007) \\
\hline & 221 & 20031213 & Stanishev et al. (2007) \\
\hline & 272 & 20040202 & Stanishev et al. (2007) \\
\hline & 377 & 20040517 & Stanishev et al. (2007) \\
\hline \multirow[t]{3}{*}{ SN 2003hv } & 113 & 20031228 & Leloudas et al. (2009) \\
\hline & 145 & 20040129 & Leloudas et al. (2009) \\
\hline & 323 & 20040725 & Leloudas et al. (2009) \\
\hline SN 2004bv & 171 & 20041114 & BSNIP \\
\hline SN 2004eo & 228 & 20050516 & Pastorello et al. (2007) \\
\hline SN 2005cf & 319 & 20060427 & Wang et al. (2009) \\
\hline \multirow[t]{9}{*}{ SN 2007af } & 103 & 20070620 & CfA \\
\hline & 108 & 20070625 & $\mathrm{CfA}$ \\
\hline & 120 & 20070707 & BSNIP \\
\hline & 123 & 20070710 & $\mathrm{CfA}$ \\
\hline & 128 & 20070715 & BSNIP \\
\hline & 131 & 20070718 & $\mathrm{CfA}$ \\
\hline & 151 & 20070807 & BSNIP \\
\hline & 165 & 20070821 & BSNIP \\
\hline & 308 & 20080111 & CfA \\
\hline
\end{tabular}


Table 1 (cont'd)

\begin{tabular}{|c|c|c|c|}
\hline SN & $\begin{array}{c}\text { Phase } t^{a} \\
\quad \text { (days) }\end{array}$ & Obs. Date ${ }^{b}$ & Spec. Ref. ${ }^{c}$ \\
\hline SN 2007gi & 161 & 20080115 & Zhang et al. (2010) \\
\hline SN 2007le & 317 & 20080827 & BSNIP \\
\hline SN 2007sr & 177 & 20080623 & CfA \\
\hline SN 2009le & 324 & 20101016 & $\mathrm{~T} 15 \mathrm{~b}$ \\
\hline \multirow[t]{2}{*}{ SN 2011by } & 206 & 20111202 & Silverman et al. (2013a) \\
\hline & 310 & 20120315 & Silverman et al. (2013a) \\
\hline \multirow[t]{6}{*}{ SN $2011 \mathrm{fe}$} & 74 & 20111123 & Shappee et al. (2013) \\
\hline & 114 & 20120102 & Shappee et al. (2013) \\
\hline & 196 & 20120324 & Shappee et al. (2013) \\
\hline & 230 & 20120427 & Shappee et al. (2013) \\
\hline & 276 & 20120612 & Shappee et al. (2013) \\
\hline & 314 & 20120720 & Taubenberger et al. (2015) \\
\hline SN 2011iv & 318 & 20121024 & $\mathrm{~T} 15 \mathrm{~b}$ \\
\hline \multirow[t]{2}{*}{ SN 2012cg } & 330 & 20130507 & M15 \\
\hline & 342 & 20130513 & $\mathrm{~T} 15 \mathrm{~b}$ \\
\hline \multirow[t]{9}{*}{ SN $2012 \mathrm{fr}$} & 101 & 20130221 & This work \\
\hline & 116 & 20130308 & This work \\
\hline & 125 & 20130317 & This work \\
\hline & 151 & 20130412 & This work \\
\hline & 222 & 20130622 & This work \\
\hline & 261 & 20130731 & This work \\
\hline & 340 & 20131018 & This work \\
\hline & 357 & 20131103 & M15 \\
\hline & 367 & 20131114 & This work \\
\hline \multirow[t]{2}{*}{ SN $2012 \mathrm{hr}$} & 283 & 20131006 & This work \\
\hline & 368 & 20131230 & This work \\
\hline \multirow[t]{6}{*}{ SN 2013aa } & 137 & 20130710 & This work \\
\hline & 185 & 20130827 & This work \\
\hline & 202 & 20130913 & This work \\
\hline & 342 & 20140131 & This work \\
\hline & 358 & 20140216 & M15 \\
\hline & 430 & 20140422 & M15 \\
\hline SN 2013cs & 320 & 20140322 & This work \\
\hline \multirow[t]{2}{*}{ SN 2013dy } & 333 & 20140626 & Pan et al. (2015a) \\
\hline & 419 & 20140920 & This work \\
\hline SN 2013gy & 276 & 20140920 & This work \\
\hline SN 2014J & 231 & 20140920 & This work \\
\hline
\end{tabular}

Note. - ${ }^{a}$ With respect to date of $B$-band peak brightness.

${ }^{b}$ Observation dates that are italicized are not used to measure $M_{N i}$, and are only employed in Section 4

c BSNIP: Silverman et al. 2012a); CfA: Matheson et al. 2008); Blondin et al. (2012); M15: Maguire et al. (2015), in preparation; T15b: Taubenberger et al. (2015b), in preparation.

\subsection{Compilation of Literature Data}

For reasons outlined in Section 4 the earliest epochs from which we can use [Co III] $\lambda 5893$ line fluxes is at phase $t=+150$ days. In practice, we found for most spectra beyond $t \approx+400$ days that the [Co III] $\lambda 5893$ flux was too weak to be usable for our preferred analysis. Furthermore, Taubenberger et al. (2015) recently showed that the $t=+1000$ day spectrum of SN 2011fe showed dramatic changes in its structure, likely arising from a change in the ionization condition of the nebula. Indeed, this ionization change appears evident in the $t=+590$ day spectrum presented in ?, and we see evidence for the onset of this change shortly after $t \approx+400$ days in the data gathered for this analysis. Thus we excise data later than $t \approx+400$ days as unreliable due to low signal and likely ioniza- tion change (we examine potential impact from the latter effect in Section 6.2.

To begin compiling a sample that meets these phase criteria, we performed a large query of the WISeREP 1 Yaron \& Gal-Yam 2012) database to search for SNe Ia with two spectroscopic observations separated by at least 100 days - assuming the earlier one would be near maximum light, this singles out SNe Ia with nebular spectra. We then require $\mathrm{SNe}$ to have photospheric-phase optical light curves sufficient to robustly establish light curve stretch, colour, and the date of maximum light using SiFTO (Conley et al. 2008). We also require the spectra to have sufficiently high signalto-noise so that the [Co III] $\lambda 5893$ line can be well fit using a Gaussian fitting procedure (see Section 3). SN 2006X was excluded (despite having numerous nebular spectra) due to significant variability in its sodium features (Patat et al. 2007) and a rather significant light echo (Wang et al. 2008a; Crotts \& Yourdon 2008), both of which might affect the time evolution of the [Co III] $\lambda 5893$ flux.

Finally, we excise any SNe Ia which are spectroscopically peculiar in the nebular phase: SNe Ia similar to SN 1991bg (Filippenko et al. 1992b; Leibundgut et al. 1993) exhibit extremely narrow $\mathrm{Fe}$ lines and unusual line ratios; Ia-CSM SNe (Silverman et al. 2013c) are excluded due to possible impact of CSM on the nebular emission; SNe Iax (Foley et al. 2013) are excised as these probably arise from a different physical mechanism than normal SNe Ia; candidate super-Chandrasekhar SNe Ia (Howell et al. 2006) are excised due to their unusual nebular spectra (Taubenberger et al. 2013). SNe Ia similar to SN 1991T (Phillips et al. 1992; Filippenko et al. 1992a) or SN 1999aa are however included in the sample, as their ionization structure appears to be similar to "normal" SNe Ia.

In summary, the selection criteria for our sample of literature nebular SN Ia spectra are:

- Phase (with respect to $B$-band maximum light) in the range $+150 \leq t \leq+400$

- Well-sampled multi-colour photospheric phase light curve (such that the light curve fitter SiFTO converges)

- Sufficient spectrum S/N to measure the [Co III] $\lambda 5893$ line center and width

- No spectroscopic peculiarity, except SN 1991T-like

The full sample of spectra which meet these criteria are presented in Table 1 and comprise 77 spectra of $25 \mathrm{SNe}$ Ia from the literature.

Finally we note that two of the SNe in our sample had prominent light echoes at late times: SN 1991T (Schmidt et al. 1994) and SN 1998bu (Spyromilio et al. 2004). For both of these SNe, the light echo contributions are negligible at the spectroscopic epochs we employ.

\subsection{New SN Ia Nebular Spectroscopy}

We obtained new late phase $(+50 \leq t \leq+150$ days $)$ and nebular ( $t \geq+150$ days) spectra of 7 nearby SNe Ia from numerous telescopes. These spectra have been released publicly on WISeREP, with several spectra of SN 2012fr released through PESSTO's ESO data releases 2 . Information about observation details are presented in Table 2 and a plot of the spectra is shown in Figure 1. We note these spectra have not been rescaled to match observed photometry.

\footnotetext{
1 http://wiserep.weizmann.ac.il

2 www.pessto.org
} 


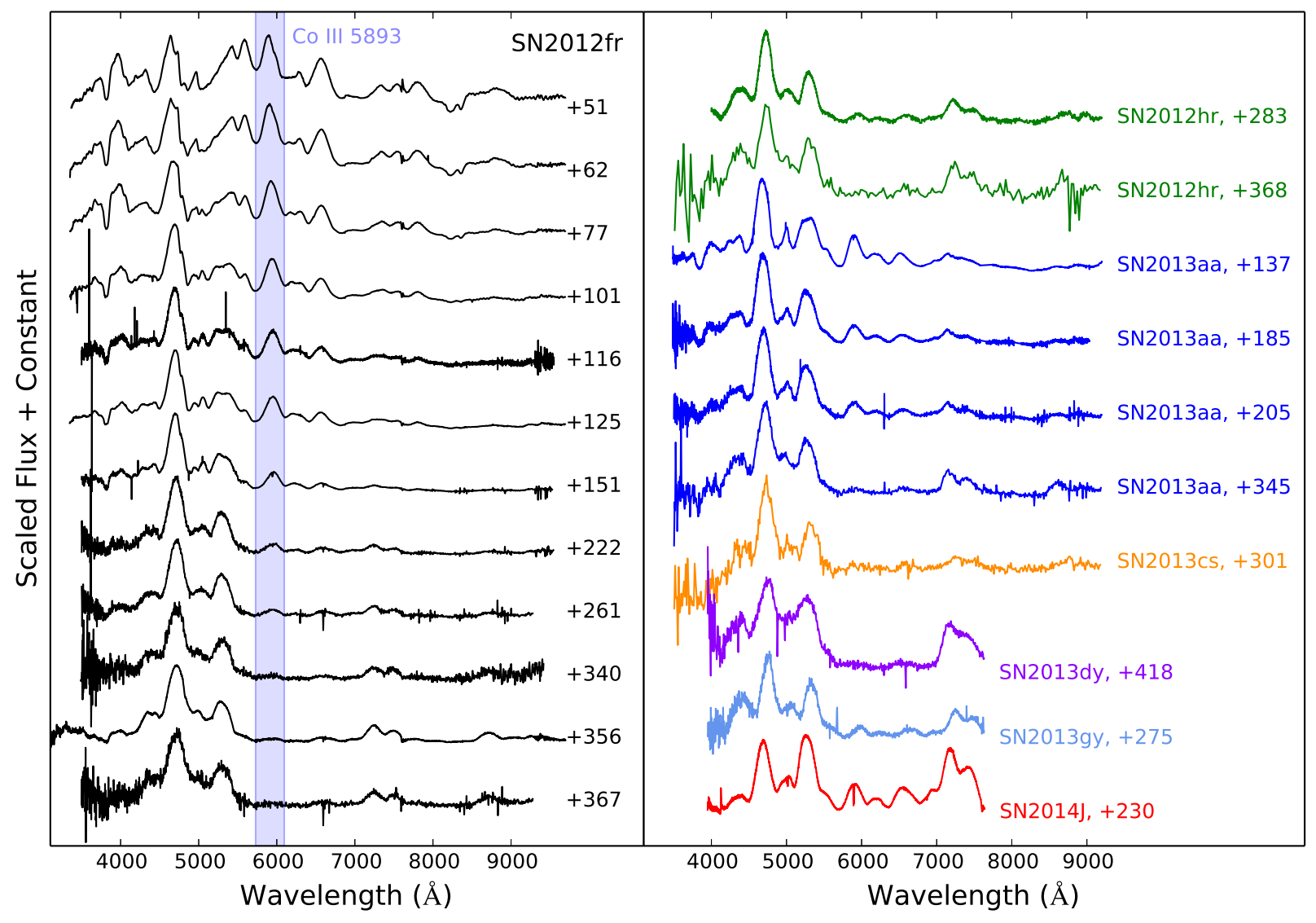

Figure 1. New late phase and nebular spectra of SNe Ia presented in this work. All spectra are publicly available on WISeREP (except the +356 day spectrum of SN 2012fr from K15). Some spectra have been slightly binned (to $\sim 5 \AA$ ) for visual clarity.

Several late phase spectra of very nearby SNe Ia were collected with the Wide Field Spectrograph (WiFeS; Dopita et al. 2007, 2010) on the ANU 2.3m telescope at Siding Spring Observatory in northern New South Wales, Australia. Observations were performed with the B3000 and R3000 gratings with the RT560 dichroic, giving wavelength range of $3500 \AA-9800 \AA$, with resolution of $0.8 \AA$ and $1.2 \AA$ on the blue and red arms, respectively. Data were reduced using the PyWiFeS package (Childress et al. 2014b), and spectra were extracted using our custom GUI (see e.g., Childress et al. 2013c). We generally observed during very dark nights (moon illumination less than 20\%) when the seeing was favorable (1". 5-2.'0). We note that the WiFeS spectra of SN $2012 \mathrm{hr}$ and SN 2013cs have too low signal-to-noise to obtain a reliable measurement of the [Co III] $\lambda 5893$ line flux, but we release them publicly (on WISeREP) here.

New nebular spectra for three nearby $\mathrm{SNe}$ Ia were collected with DEIMOS (Faber et al. 2003) on the Keck-II telescope on Mauna Kea, Hawaii. Observations were conducted with a $1^{\prime \prime} .5$ longslit, the 600 1/mm grating with a central wavelength of $5000 \AA$ and with the GG410 order blocking filter, yielding a wavelength range of $4000 \AA-7650 \AA$ with $0.6 \AA$ resolution. Data were reduced using standard techniques in IRAF (see e.g., Childress et al. 2013a), with the blue and red chips reduced separately then combined as a final step. We employed the Mauna Kea extinction curve of Buton et al. (2013). Our observations come from a single night on Keck (2014-Sep-20 UTC) when conditions were less favorable (high humidity and thick clouds, ultimately 50\% time lost to weather) but with a median seeing of 0.9 .

Five additional late phase spectra of SN 2012fr were collected as part of the Public ESO Spectroscopic Survey of Transient Objects (PESSTO; Smartt et al. 2015) during early 2013, and reduced with the PESSTO pipeline as described in Smartt et al. (2015). One spectrum of SN 2012fr and two spectra of SN 2013aa were obtained in 2013 using the Robert Stobie Spectrograph on the South African Large Telescope (SALT), and reduced using a custom pipeline that incorporates PyRAF and PySALT (Crawford et al. 2010). One spectrum of SN 2012hr was obtained with Gemini GMOS (Hook et al. 2004) using the $0.75^{\prime \prime}$ longslit with the B600 and R400 gratings in sequence to yield a spectral coverage from 4000 - $9600 \AA$, under program GS-2013B-Q-48 (PI: Graham) the spectrum was reduced using the Gemini IRAF package.

In the analysis below we also include nebular spectroscopy samples from forthcoming analyses by Maguire et al. (2015, in prep. - hereafter M15) and Taubenberger et al. (2015b, in prep. - hereafter T15b). The M15 sample were obtained over a multiperiod program at the VLT using XShooter (Vernet et al. 2011), and were reduced with the XShooter pipeline (Modigliani et al. 2010) using standard procedures (as in Maguire et al. 2013). The T15b sample were observed as part of a separate multi-period program 
Table 2. Observation details for new late phase SN Ia spectra

\begin{tabular}{lccc}
\hline SN & $\begin{array}{c}\text { Phase } \\
\text { (days) }\end{array}$ & $\begin{array}{c}\text { Obs. } \\
\text { Date }\end{array}$ & $\begin{array}{c}\text { Telescope } \\
\text { / Instrument }\end{array}$ \\
\hline SN 2012fr & +51 & 2013-Jan-02 & NTT-3.6m / EFOSC \\
& +62 & 2013-Jan-13 & NTT-3.6m / EFOSC \\
& +77 & 2013-Jan-28 & NTT-3.6m / EFOSC \\
& +101 & 2013-Feb-21 & NTT-3.6m / EFOSC \\
& +116 & 2013-Mar-08 & ANU-2.3m / WiFeS \\
& +125 & 2013-Mar-17 & NTT-3.6m / EFOSC \\
& +151 & 2013-Apr-12 & ANU-2.3m / WiFeS \\
& +222 & 2013-Jun-22 & ANU-2.3m / WiFeS \\
& +261 & 2013-Jul-31 & ANU-2.3m / WiFeS \\
& +340 & 2013-Oct-18 & SALT / RSS \\
SN 2012hr & +367 & 2013-Nov-14 & ANU-2.3m / WiFeS \\
& +283 & 2013-Oct-06 & Gemini / GMOS \\
SN 2013aa & +368 & 2013-Dec-30 & ANU-2.3m / WiFeS \\
& +187 & 2013-Jul-10 & SALT / RSS \\
& +202 & 2013-Aug-27 & SALT / RSS \\
& +342 & 2014-Jep-13 & ANU-2.3m / WiFeS \\
SN 2013cs & +320 & 2014-Mar-22 & ANU-2.3m / WiFeS \\
SN 2013dy & +419 & 2014-Sep-20 & Keck-II / DEIMOS \\
SN 2013gy & +276 & 2014-Sep-20 & Keck-II / DEIMOS \\
SN 2014J & +231 & 2014-Sep-20 & Keck-II / DEIMOS \\
\hline
\end{tabular}

using FORS2 on the VLT, and data were reduced with standard procedures similar to those employed in Taubenberger et al. (2013).

\section{NEBULAR LINE FLUX MEASUREMENTS}

\subsection{The [Co III] $\lambda 5893$ line in the nebular phase: a radiative transfer perspective}

The current study was motivated by the disappearance of Co III lines in nebular time series spectra, most notably the feature near $5900 \AA$ A. Previous literature analyses have attributed this feature alternately to Co III and $\mathrm{Na}$ I, so we turned to radiative transfer calculations to settle this ambiguity.

We employed the time-dependent radiative transfer code CMFGEN (Hillier \& Dessart 2012), which solves the time dependent radiative transfer equation simultaneously with the kinetic equations. Given an initial explosion model, we self-consistently solve for the temperature structure, the ionization structure, and the non-LTE populations, beginning the calculations at 0.5 to 1 day after the explosion. The modelling assumes homologous expansion, typically uses a $10 \%$ time step, and no changes are made to the ejecta structure (other than that required by homologous expansion) as the ejecta evolve in time. Further details about the general model set up, and model atoms, can be found in Dessart et al. (2014c). We deployed CMFGEN on a delayed-detonation model (DDC10 Blondin et al. 2013; Dessart et al. 2014c) at very late phases and examine the contribution of various ions to the nebular emission spectrum. Radiative transfer calculations for this model, and similar models but with a different initial ${ }^{56} \mathrm{Ni}$ mass, have shown favorable agreement with observations (Blondin et al. 2013, 2015; Dessart et al. 2014c b. a).

In Figure 2 we show DDC10 modeled with CMFGEN at phases +126 days (left panels) and +300 days (right panels). The top panels in each column show the integrated DDC10 model flux compared to observations of nebular phase $\mathrm{SNe}$ Ia at similar phases, while the bottom panels show the line emission from individual ions (note this can exceed the integrated flux due to the net opacity encountered by photons following their initial emission). The +126 day model shows particularly good agreement with the data. At +300 days the model shows some discrepancy with the data, particularly in the ionization state of the nebula.

Most importantly, the radiative transfer calculations show that the emission feature near $5900 \AA$ is clearly dominated by Co III emission, with little or no contamination from other species. Few other features in the optical region of the spectrum show such clean association with a single ion.

For later aspects of our analysis we require the velocity center of the nebula, which we calculate from the [Co III] $\lambda 5893$ line. To do so requires an accurate calculation of the mean rest wavelength for this line complex. The [Co III] $\lambda 5893$ arises from the $3 \mathrm{~d}^{7} \mathrm{a}^{4} \mathrm{~F}-3 \mathrm{~d}^{7} \mathrm{a}^{2} \mathrm{G}$ multiplet, and is actually a blend of two lines one at $5888.5 \AA$ and a second, but weaker, line at $5906.8 \AA$ (see Appendix A and Table A1 . Given the $A$ values and wavelengths of the transitions contributing to the line complex, the weighted mean rest wavelength of the Co III line is $5892.7 \AA$ (note: this and previous are air wavelengths). Henceforth we use this value for calculating line velocities.

\subsection{Measuring the [Co III] $\lambda 5893$ line flux}

For the main analyses in this work we focus on the flux in the [Co III] $\lambda 5893$ line. We measure the flux in this line as follows.

We perform an initial Gaussian fit to the [Co III] $\lambda 5893$ line in order to the determine the center and width of the line. We then integrate the flux within $\pm 1.5 \sigma$ of the fitted line center and use this "integral" flux for the remainder of this paper. This integral boundary was chosen as a compromise between capturing a large fraction of the emitted line flux ( $97 \%$ for a strictly Gaussian profile) and limiting contamination from neighbouring emission lines. For $\mathrm{SNe}$ Ia with multiple nebular spectra we enforce common wavelength bounds for the flux integration at all epochs, as determined by the median fitted line center and width values across all epochs. Generally the integrated line flux and that calculated from the best Gaussian fit showed excellent agreement (see Figure 3), but we prefer the integral flux as this is robust against non-Gaussianity of the line profile.

To place our [Co III] $\lambda 5893$ line flux measurements on the correct absolute scale, we must ensure the spectra have the correct absolute flux calibration. To achieve this, we measure the expected $B$-band flux in the spectrum by convolving it with the $B$-band filter throughput curve and integrating. We then compute the ratio of this flux collected in the spectrum $B$ passband to the true $B$-band flux of the SN at that epoch. The latter is determined from the late-time photometry for each of our SNe, as outlined in Appendix B and presented in Table B3. To ensure reproducability of our results, we report in this table the flux values derived from the raw measurements made from the spectra in their published form.

We note that normalization with the $B$-band could introduce errors in the [Co III] $\lambda 5893$ flux due to chromatic errors in the spectrum's flux calibration. However, previous authors consistently performed chromatic flux calibration using spectrophotometric standard stars, typically yielding excellent colour agreement with observed photometry (e.g. $B-V$ scatter of 0.08 mag and 0.10 mag for the CfA and BSNIP samples, repectively). We also note that other systematic effects could affect our measurements of [Co III] $\lambda 5893$ line flux. These include contamination from neighboring nebular emission lines (e.g. Fe II lines, see Figure 2), residual host galaxy 

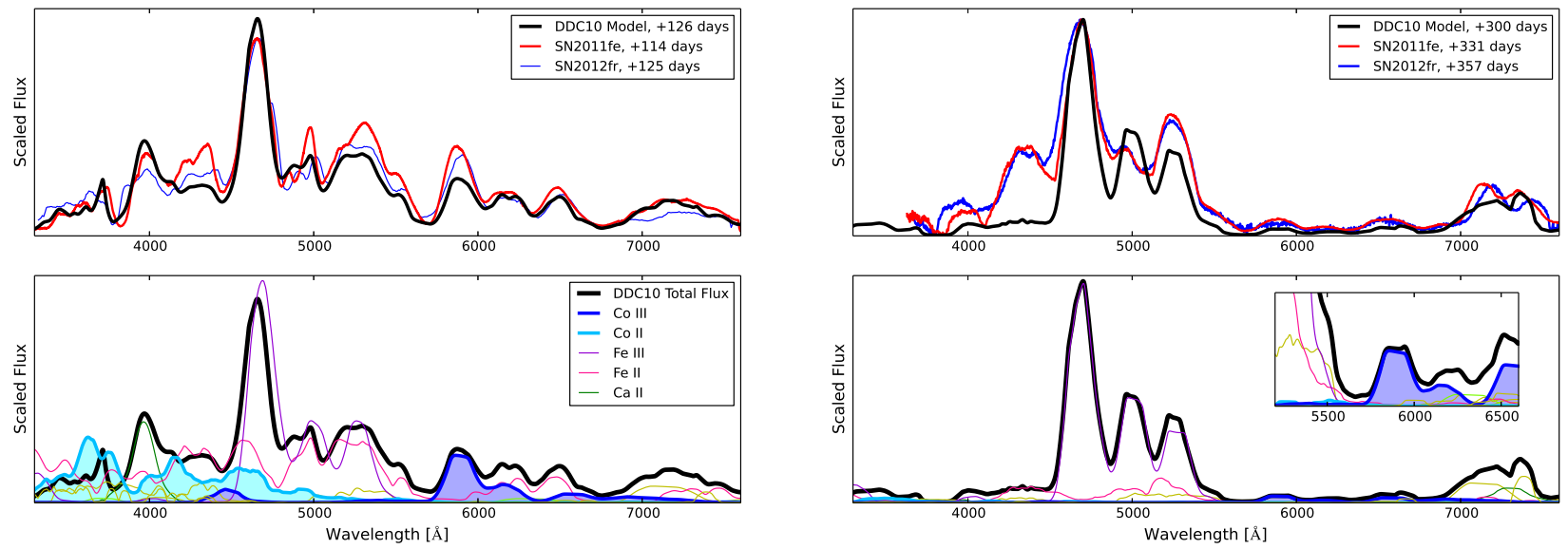

Figure 2. Top panels: Comparison of radiative transfer (CMFGEN) model spectrum for the DDC10 (Blondin et al. 2013) delayed detonation model at very late epochs (left: +126 days, right: +300 days) compared to contemporaneous data for SN 2011fe and SN 2012fr. Bottom panels: Emission spectra for various ions from CMFGEN for late-phase DDC10 models (epochs as above).

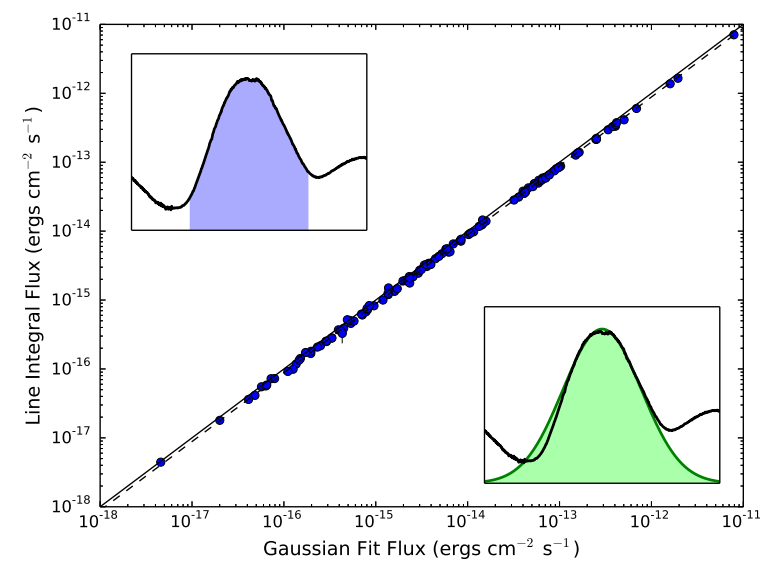

Figure 3. Comparison of flux in the [Co III] $\lambda 5893$ line measured in two ways: strict integration of the spectrum flux within $\pm 1.5 \sigma$ of the fitted line center (y-axis and upper left inset), and the formal integral of the best fit Gaussian profile (x-axis and lower right inset). The solid line represents unity, while the dashed line is the mean ratio of the integral flux to Gaussian flux for the full sample $(0.87 \pm 0.05)$.

light, or perhaps even previously undetected light echoes (see, e.g., Spyromilio et al.2004). Thus we expect a conservative estimate for the systematic uncertainty in the [Co III] $\lambda 5893$ flux measurement to be about $10 \%$ of the measured flux.

The final integrated [Co III] $\lambda 5893$ line flux, wavelength bounds for the integral, and synthetic $B$-band flux integrated from the spectrum are all presented in Table B4 Variance spectra were not available for many of the literature SN Ia spectra in our analysis. To correct this, we smooth the spectrum with a Savitszky-Golay filter (Savitzky \& Golay 1964), then smooth the squared residuals of the data from this smooth curve to derive a variance spectrum measured directly from the noise in the data (as we did for data in Childress et al. 2014a). [Co III] $\lambda 5893$ line flux errors were then determined from these corrected variance spectra.

\section{EVOLUTION OF THE [Co III] $\lambda 5893$ LINE FLUX}

\subsection{Theoretical expectations for [Co III] $\lambda 5893$ evolution}

The decay of ${ }^{56} \mathrm{Co}$ to ${ }^{56} \mathrm{Fe}$ produces positrons and energetic gamma-rays. The charged positrons carry kinetic energy which they lose to the surrounding medium via Coulomb interactions. At the nebular densities present at late times, the length scale for positron energy deposition is much smaller than the size of the nebula so the positrons essentially deposit all of their kinetic energy locally (Chan \& Lingenfelter 1993). gamma-rays either those emitted directly from ${ }^{56} \mathrm{Co}$ decay or created when the positrons annihilate - are subject to radiative transfer effects and will eventually free stream as the SN nebula expands and decreases its density enough to become optically thin to gammarays. The onset of this phase - where positrons deposit a constant fraction of energy into the SN nebula and gamma-rays escape fully - has been observed in late SN Ia bolometric light curves (e.g. Sollerman et al. 2004; Stritzinger \& Sollerman 2007; Leloudas et al. 2009; Kerzendorf et al. 2014b).

Our expectation from a simple energetics perspective is that the flux of the [Co III] $\lambda 5893$ line should evolve as the square of the mass of cobalt as a function of time $M_{C o}(t)$. The energy being deposited into the nebula at these late phases arises from the positrons produced in ${ }^{56} \mathrm{Co}$ decay, and thus should scale with the mass of cobalt. If this energy is evenly deposited amongst all species in the nebula then the fraction of that energy absorbed by the cobalt atoms should be proportional to the mass fraction of cobalt. Thus the amount of energy absorbed by cobalt atoms follows the square of the cobalt mass as a function of time. If the fraction of that energy emitted in the [Co III] $\lambda 5893$ line remains constant (see Section 6.2 then we expect a net quadratic dependence of the [Co III] $\lambda 5893$ line luminosity on the mass of cobalt as a funciton of time.

Observational evidence for this temporal evolution of the [Co III] $\lambda 5893$ line should be expected from prior results. The latephase bolometric light curves of SNe Ia closely follow the amount of energy deposited by the decay of ${ }^{56} \mathrm{Co}$ (see, e.g., Sollerman et al. 2004). It was also demonstrated by Kuchner et al. (1994) that the ratio of [Co III] $\lambda 5893$ to $\mathrm{Fe} 4700$ emission follows the $\mathrm{Co} / \mathrm{Fe}$ mass ratio (as noted above), and the Fe 4700 line flux generally scales with the total luminosity of the $\mathrm{SN}$ since $\mathrm{Fe}$ is the primary 
coolant. These facts combine to lend an expectation that the net emission from the [Co III] $\lambda 5893$ line should scale quadratically with the mass of Co in the SN nebula as a function of time. Indeed, McClelland et al. (2013) found such a quadratic dependence for the [Co III] $\lambda 5893$ line in SN $2011 \mathrm{fe.}$

The above reasoning for $M_{C o}^{2}$ dependence of the [Co III] $\lambda 5893$ flux holds for epochs when the nebula is fully transparent to gamma-rays. Thus it is important to inspect the theoretical expectation for the timing of this gamma-ray transparency in the IGE zone. The energy released per decay from ${ }^{56} \mathrm{Co}$ is $3.525 \mathrm{MeV}$, of which $3.3 \%$ is associated with the kinetic energy of the positrons, and we have ignored the energy associated with neutrinos. As the expansion is homologous, the optical depth associated with gamma-rays scales as $1 / t^{2}$. Assuming that the kinetic energy of the positrons is captured locally, the energy absorbed per ${ }^{56} \mathrm{Co}$ decay in $\mathrm{MeV}$ is

$$
e_{C o}=0.116+3.409\left(1-\exp \left[-\tau_{o}\left(t_{o} / t\right)^{2}\right]\right)
$$

where $\tau_{o}$ is the effective optical depth at a time $t_{o}$. If we denote $t_{c}$ as the time at which energy deposition by gamma-rays and positrons are equal, then Equation 1 can be rewritten as:

$$
E_{C o} \propto M_{C o}\left(1-0.967 \exp \left[-0.0346\left(t_{c} / t\right)^{2}\right]\right)
$$

We expect the flux from the [Co III] $\lambda 5893$ line would further scale as:

$$
F_{C o} \propto E_{C o} \times \frac{x M_{C o} / M_{I G E}}{1+(a-1) M_{C o} / M_{I G E}+b M_{O t} / M_{I G E}}
$$

where $M_{I G E}$ is the total mass of the IGE zone, $a$ and $b$ are respectively the (time-dependent) factors relating the cooling efficiency of Co and other species (which have total mass of $M_{O t}$ ) relative to iron, and $x$ is the factor scaling the emission in the [Co III] $\lambda 5893$ feature. If the thermal conditions in the $\mathrm{SN}$ nebula are relatively stable (i.e. constant $x$ ) and cooling by non-iron species is negligible (i.e. the above denominator goes to unity), then the line flux simply becomes proportional to $M_{C o} / M_{I G E}$. Combining Equations 2 and 3 yields:

$$
F_{C o} \propto M_{C o}^{2}\left(1-0.967 \exp \left[-0.0346\left(t_{c} / t\right)^{2}\right]\right)
$$

For the DDC10 model, we find $t_{c} \sim 214$ days (from explosion) - this would imply a deviation from $M_{C o}^{2}$ of a factor of 2 from +150 to +400 days past maximum light (assuming a rise time of $\sim 17$ days), or a factor of 1.5 from +200 to +400 days. Alternatively if $t_{c} \sim 80$ days (see Section 4.3 then the deviation from $M_{C o}^{2}$ is only $20 \%$ from +150 to +400 days and $10 \%$ from +200 to +400 days.

\subsection{Observed [Co III] $\lambda 5893$ evolution in nebular spectral time series}

To examine the observed evolution of the [Co III] $\lambda 5893$ line, we turn to those SNe Ia with numerous nebular spectra. Specifically, we isolate the subset of SNe Ia in our sample with at least three epochs of observation later than +150 days past maximum. For the eight SNe Ia in our sample which meet this criterion, we also collect spectra between $+100 \leq t \leq+150$ days past maximum (dates listed in italics in Table 1). These additional spectra allow us to further inspect the [Co III] $\lambda 5893$ flux evolution, but these spectra are not employed in our nickel mass estimates derived in Section 5

In the upper panel of Figure 4 we show the evolution of the [Co III] $\lambda 5893$ line luminosity versus time for our sample of $\mathrm{SNe}$ Ia with three or more observations after +150 days. We plot the line evolution for a linear (dotted) line and quadratic (solid
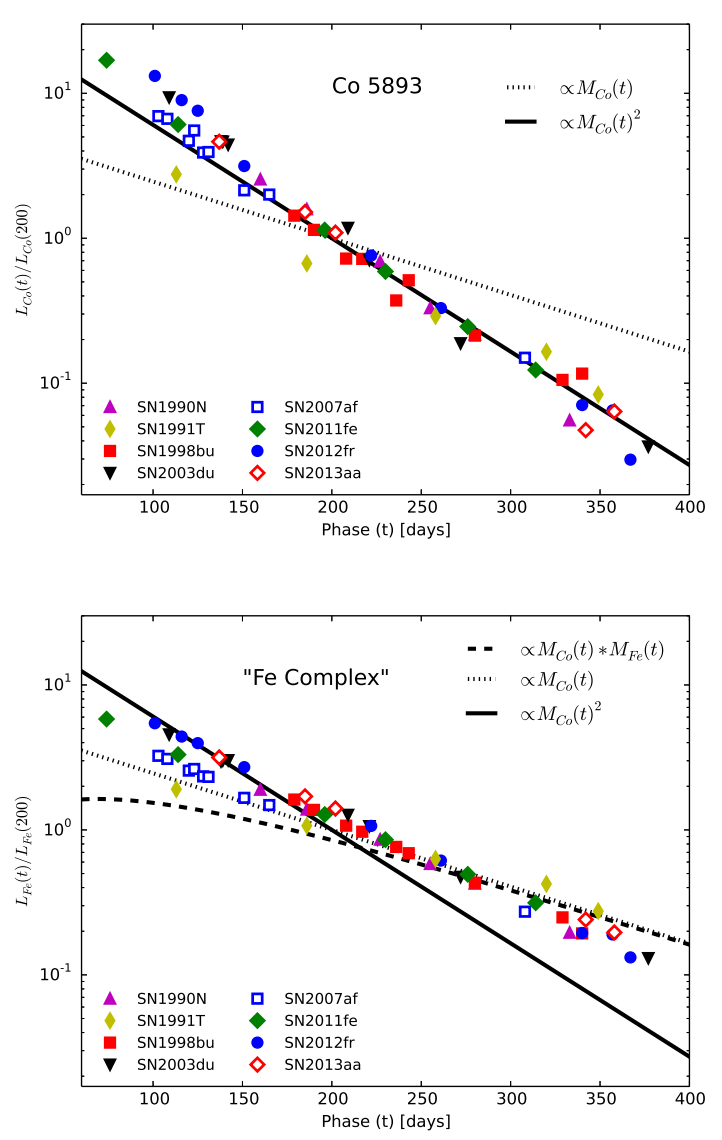

Figure 4. Top: Evolution of the [Co III] $\lambda 5893$ line flux in SNe Ia with nebular time series ( $\geq 3$ observations past 150 days), compared to curves following the mass of ${ }^{56} \mathrm{Co}$ as a function of time to the first power (dotted line) and second power (solid line). Data for each SN was shifted by a multiplicative offset (i.e. $\log$ additive offset) that reduced residuals with the $M_{C o}(t)^{2}$ line. Bottom: Evolution of the "Fe complex" flux with phase, compared to the same lines as above as well as an additional line proportional to the product of the ${ }^{56} \mathrm{Co}$ mass with the ${ }^{56} \mathrm{Fe}$ mass as a function of time (dashed curve).

line) dependence on $M_{C o}(t)$, with both curves normalized at phase $t=+200$ days. For each $\mathrm{SN}$ in this subset, we fit for a single multiplicative scaling factor that minimizes the residuals of the $M_{C o}(t)^{2}$ line (i.e. we normalize each SN data set to that line - thus the reason for requiring multiple data points per $\mathrm{SN}$ ). This isolates the time dependence of the line flux (which depends on the SN nebula physics) by removing its absolute magnitude (which depends on the quantity of ${ }^{56} \mathrm{Ni}$ produced).

The evolution of the [Co III] $\lambda 5893$ line shows a remarkable agreement with the expected trend of $M_{C o}(t)^{2}$, perhaps as early as phase +150 days. The one possible exception to the $M_{C o}(t)^{2}$ trend is SN 1991T, which appears to have a shallower evolution than the other SNe Ia. As we show below (Section4.3), this cannot arise from gamma-ray opacity. Instead the most likely explanation is probably a higher ionization state at early epochs ( $t \leq 300$ days). Because of this, for SN 1991T only we excise epochs prior to 300 days when calculating its ${ }^{56} \mathrm{Ni}$ mass in Section 5.2 - a choice which yields more favorable agreement with previous analyses from the literature.

To contrast the behavior of the [Co III] $\lambda 5893$ line with other 
regions of the nebular spectra, we also inspected the evolution of the blue "Fe complex" of lines. For each spectrum we integrate the flux in the region $4100-5600 \AA$ (adjusted for each SN according to its central nebular velocity measured from the Co line) where the emission is almost entirely dominated by Fe lines (see Figure 2). Following our arguments for the expectation of the [Co III] $\lambda 5893$ line flux, the Fe complex flux should be proportional to the energy being deposited - which scales as $M_{C o}(t)$ - and the mass fraction of $\mathrm{Fe}$ (which should be relatively constant as $M_{C o} \ll M_{F e}$ at this point). Thus the Fe complex flux should scale linearly with $M_{C o}(t)$. In the lower panel of Figure 4 we plot the evolution of the Fe flux for the sample of SNe Ia, and see that it follows more closely the $M_{C o}(t)$ curve than the $M_{C o}(t)^{2}$ curve. However, we do note deviation from this line such that the logarithmic slope is somewhat intermediate between 1 and 2. Additionally, earlier epochs are subject to a complicated interplay of additional energy deposition from gamma-rays (as for the [Co III] $\lambda 5893$ line, see Section 4.3, decreased emission due to nonzero optical depth in this region of the spectrum, and possible emission from Co II (see Figure 2).

We note that the above results also explain one aspect of the data presented in Förster et al. (2013). Those authors examined the late (35 $\lesssim t \lesssim 80$ days) colour evolution (i.e. Lira law) for a large sample of nearby SNe Ia and its relationship with dust absorption (as inferred from narrow sodium absorption). The mean value of $B$ band decline rates were roughly $0.015 \mathrm{mag} / \mathrm{day}$, while the V-band decline rates were nearly twice that $(0.030 \mathrm{mag} / \mathrm{day})$. The $B$-band is dominated by the Fe complex whose flux decays as $M_{C o}(t)$, while the V-band is heavily influenced by Co lines (see Figure 2 in Section 3.1 whose flux decays as $M_{C o}(t)^{2}$. This naturally explains why the luminosity decay rate (in mag/day) in V-band is nearly twice that of the $B$-band, and contributes to why SNe Ia become bluer (in $B-V$ ) with time at these epochs.

\subsection{Testing gamma-ray opacity effects on [Co III] $\lambda 5893$ evolution}

While the data appear to agree with an $M_{C o}(t)^{2}$ dependence of the [Co III] $\lambda 5893$ flux evolution, it is important to investigate the impact of gamma-ray energy deposition on deviation from this parametrization.

To this end, we isolated the subset of $\mathrm{SNe}$ Ia from our sample with at least one nebular spectrum earlier than +150 days and at least one spectrum later than +250 days. For the six SNe Ia satisfying these criteria, we fit the [Co III] $\lambda 5893$ flux evolution using the parametrization of Equation 4 This fit has two free parameters: a multiplicative scaling for all the line fluxes, and the gamma-ray "crossing" time $t_{c}$ when energy deposition from gamma-rays and positrons are equal. These fits are shown in Figure 5

In general the [Co III] $\lambda 5893$ evolution is extremely well fit by this model, especially for $\mathrm{SNe}$ Ia with good temporal coverage and high signal-to-noise data (notably SN 2011fe and SN 2012fr). Some $\mathrm{SNe}$ Ia have a gamma-ray crossing time similar to the prediction from our model ( $t_{c} \sim 200$ days) while some other SNe Ia have shorter crossing times ( $t_{c} \sim 80$ days). The implications of this for SN Ia progenitors will be discussed in further detail in Section 6.1 Given these gamma-ray opacity model fit results, we calculate that deviations of [Co III] $\lambda 5893$ flux evolution from the simple $M_{C o}(t)^{2}$ could range from $15 \%$ to $100 \%$ at $t=150$, and $7 \%$ to $55 \%$ at $t=200$, and $4 \%$ to $30 \%$ at $t=250$ days.
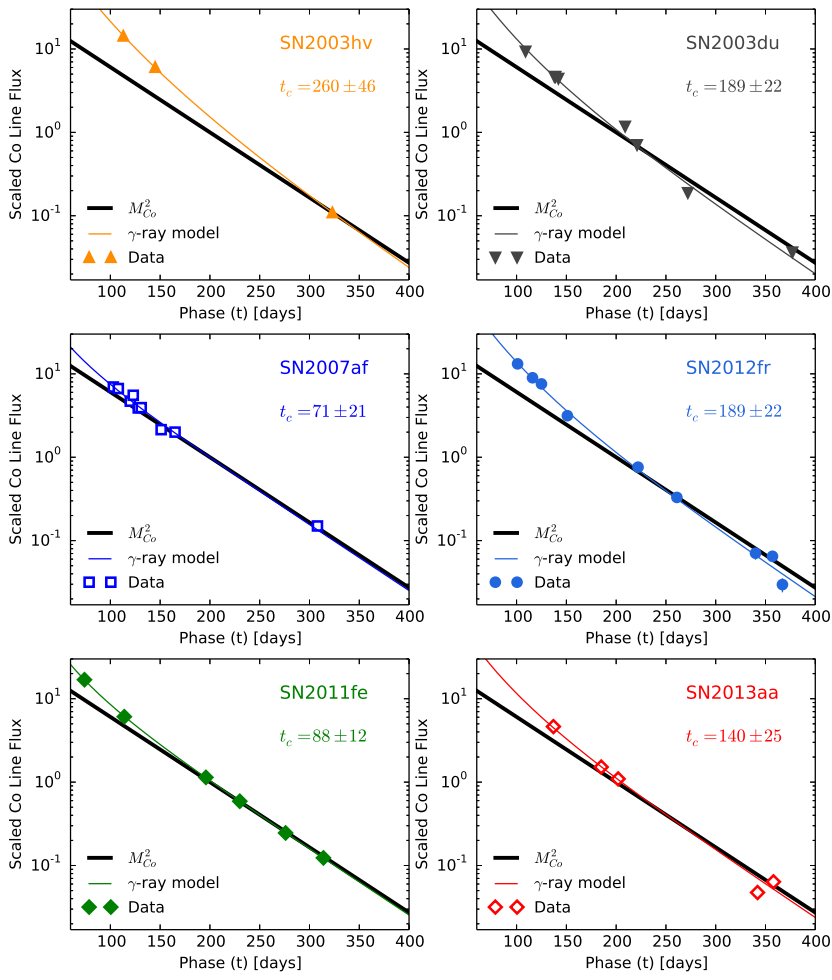

Figure 5. Fits of gamma-ray opacity model to select SN Ia [Co III] $\lambda 5893$ line fluxes. The fitted "crossing" time (when gamma-ray and positron energy deposition are equal) is shown in each panel.

\section{MEASURING ${ }^{56}$ NI MASS FROM SN Ia NEBULAR SPECTRA}

\subsection{Placing [Co III] $\lambda 5893$ flux measurements at disparate epochs on a common scale}

To place all our SN Ia [Co III] $\lambda 5893$ fluxes on a common scale, we first convert the observed line flux to the absolute line luminosity emitted by the SN using the distance to the SN host galaxy. For some SNe Ia in our sample, redshift-independent distance measurements exist for the host galaxy, particularly a number with Cepheid distance measurements. For most of the SNe Ia in our sample, however, the SN distance is computed by converting the host galaxy redshift to a distance using a Hubble constant value of $H_{0}=73.8 \mathrm{~km} \mathrm{~s}^{-1} \mathrm{Mpc}^{-1}$ chosen from Riess et al. (2011) to maintain consistency with those hosts with Cepheid distances from that work. For hosts with redshift-based distances, we assign a distance uncertainty corresponding to a peculiar velocity uncertainty of $300 \mathrm{~km} \mathrm{~s}^{-1}$. Table B1 lists the full set of distance moduli (and references) employed in our sample.

Calculating the absolute [Co III] $\lambda 5893$ flux emitted by each $\mathrm{SN}$ also requires correction for extinction by interstellar dust in the SN host galaxy. We accomplish this by calculating the Cardelli et al. (1989, hereafter CCM) reddening curve at the rest central wavelength of the [Co III] $\lambda 5893$ complex for an appropriate value of the reddening $E(B-V)$ and selective extinction $R_{V}$. For most $\mathrm{SNe}$ Ia in our sample, the reddening is extremely low $(E(B-V) \leq 0.10 \mathrm{mag})$, so we use the light curve colour fitted by SiFTO (Conley et al. 2008), and a selective extinction value of $R_{V}=2.8$ (appropriate for cosmological $\mathrm{SNe}$ Ia, see 


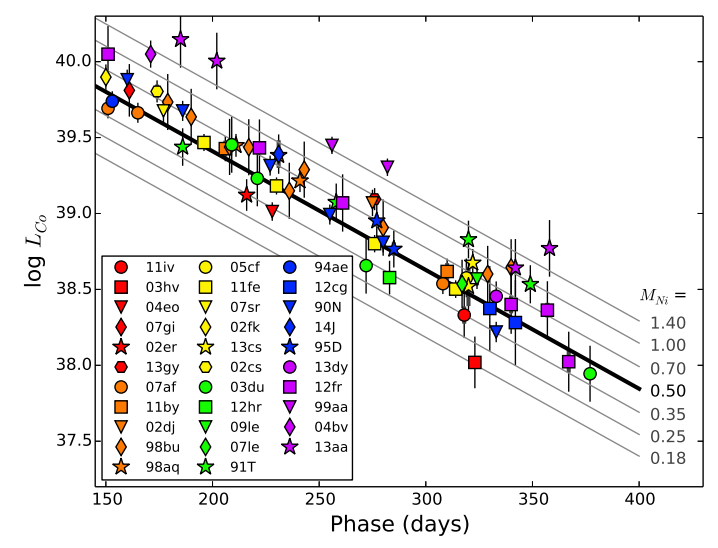

Figure 6. Evolution of the absolute [Co III] $\lambda 5893$ line luminosity as a function of phase for all SNe Ia in our sample. The solid line corresponds to the square of the mass of ${ }^{56} \mathrm{Co}$ as a function of time, anchored by the [Co III] $\lambda 5893$ luminosity for SN $2011 \mathrm{fe}$ at $\sim 200$ days. Here thick error bars correspond to flux measurement errors, while narrow error bars correspond to distance uncertainties.

Chotard et al. 2011). We note that the choice of $R_{V}$ has negligible impact on the majority of our sample. SN Ia light curve colours are affected by both intrinsic colour and host galaxy extinction (see, e.g., Scolnic et al. 2014), so for SNe Ia with negative SiFTO colours - indicating blue intrinsic colours - we apply no colour correction (i.e. colour corrections never redden the data). In this work, we are not trying to standardize SN Ia (in which applying a colour correction to the intrinsic colours may also be appropriate); rather we are only concerned with eliminating the effects of dust extinction.

Two SNe Ia in our sample, however, have strong extinction by unusual dust and thus must be treated differently. SN 2014J occurred behind a thick dust lane in the nearby starburst galaxy M82. Foley et al. (2014) performed a detailed fit of multi-colour photometry of the $\mathrm{SN}$, and find it is best fit by a CCM-like reddening curve with $E(B-V)=1.19$ and $R_{V}=1.64$. We adopt their colour correction for SN 2014J, and for the line flux uncertainty arising from the reddening correction we adopt their uncertainty for the visual extinction of $\sigma_{A_{V}}=0.18$ mag. SN 2007le showed moderately low extinction but with some variability in the sodium absorption feature likely arising from interaction of the $\mathrm{SN}$ with its circumstellar medium (Simon et al. 2009). Despite this variability, most of the absorption strength remains stable, so we adopt a colour correction for SN 2007le with $E(B-V)=0.277$ and $R_{V}=2.56$ as derived by Simon et al. (2009).

Figure 6 presents the total emitted [Co III] $\lambda 5893$ luminosity as a function of phase for all nebular spectra in our final sample. In this and subsequent figures, the thick errorbars represent the composite measurement errors from the [Co III] $\lambda 5893$ flux, $B$-band flux in the spectrum, observed (photometric) $B$-band magnitude, and extinction correction; the narrow error bars represent the distance uncertainties. Points are colour-coded (in groups) based on the light curve stretch.

The line luminosity values are then used to compute an effective luminosity of the [Co III] $\lambda 5893$ line at a common phase of +200 days for all SNe Ia in the sample (hencefoward we refer to this as $L_{C o}$ ) using the $M_{C o}^{2}$ curve. For a single nebular spectrum,

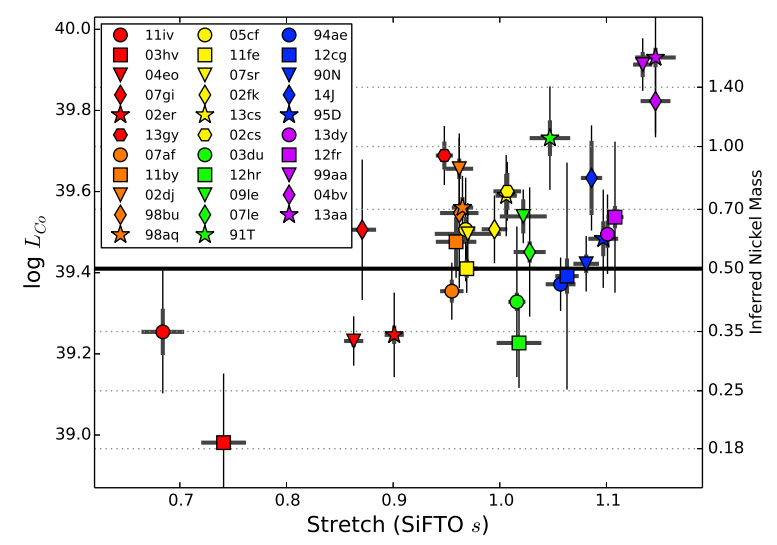

Figure 7. [Co III] $\lambda 5893$ line luminosity scaled to its equivalent value at $t=200$ days using the $M_{C o}(t)^{2}$ curve $\left(L_{C o}\right)$ versus SN light curve stretch. As in Figure 6 thick error bars correspond to flux measurement errors, while narrow error bars corresponding to distance uncertainty arising from peculiar velocities.

this can be calculated directly as:

$$
\log \left(L_{C o}(200)\right)=\log \left(L_{C o}(t)\right)+7.80 \times 10^{-3} *(t-200)
$$

For SNe Ia with multiple spectra, $L_{C o}$ is calculated as the $\chi^{2}$ weighted mean value across all acceptable epochs $(150 \leq t \leq$ 400 days) using the above equation. We note the above equation is calculated assuming a time between explosion and $B$ band peak (i.e. rise time) of 17 days, but there may be an associated uncertainty on this due to diversity in SN Ia rise times (Ganeshalingam et al. 2010) and possible dark phase before first light escapes (Piro \& Nakar 2013). Each day of difference in explosion date results in a corresponding change in the final [Co III] $\lambda 5893$ luminosity of $1.8 \%$ - assuming an explosion date uncertainty of about 3 days, we thus expect the explosion date uncertainty contributes about $5 \%$ uncertainty to the final nickel mass derived in Section 5.2

As noted in Section 4 SN 1991T may represent a case where the stable ionization state is not established until later than other $\mathrm{SNe}$ (also evident in Figure 6, so for this SN we use the later two epochs $(t \geq 300)$ to establish $L_{C o}$. This also yields a favorable agreement of our ${ }^{56} \mathrm{Ni}$ mass with literature estimates (see Section 5.2.

In Figure 7 we show the scaled $t=200 \mathrm{~d}$ [Co III] $\lambda 5893$ line luminosity plotted against light curve stretch. A clear correlation is evident between the [Co III] $\lambda 5893$ line luminosity and stretch - this is expected given the [Co III] $\lambda 5893$ luminosity traces the amount of ${ }^{56} \mathrm{Ni}$ produced in the explosion, and ${ }^{56} \mathrm{Ni}$ directly powers the peak luminosity which correlates with the light curve stretch.

\subsection{Inferring $M_{N i}$ from [Co III] $\lambda 5893$ flux}

Scaling the [Co III] $\lambda 5893$ flux values to the same phase $(t=$ 200 days) effectively places all measurements at the same epoch since explosion, so the amount of ${ }^{56} \mathrm{Co}$ will have the same proportionality to the amount of ${ }^{56} \mathrm{Ni}$ produced in the explosion. The final critical ingredient for inferring ${ }^{56} \mathrm{Co}$ mass (and thus ${ }^{56} \mathrm{Ni}$ mass) from the [Co III] $\lambda 5893$ line flux is the scaling between ${ }^{56} \mathrm{Co}$ mass and [Co III] $\lambda 5893$ flux. For reasons we will explore in Section 6.2 
we expect this conversion factor to be relatively stable in time (for phases $150 \leq t \leq 400$ days considered here) and consistent across all SNe Ia. At these phases we also expect ${ }^{56}$ Co to be the dominant isotope (by mass) of cobalt (Seitenzahl et al. 2009b), as ${ }^{57}$ Co only dominates energy deposition around $t \sim 1000$ days (Graur et al. 2015).

We expect the [Co III] $\lambda 5893$ line flux at phase $t=200$ days to be linearly proportional to the mass of ${ }^{56} \mathrm{Ni}$ produced in explosion (since the ${ }^{56} \mathrm{Co}$ mass fraction at this same epoch is necessarily the same for all SNe Ia). To convert [Co III] $\lambda 5893$ flux to ${ }^{56} \mathrm{Ni}$ mass requires some scaling between the two quantities to be determined. In principle this could be computed through radiative transfer modelling of late phases for SN Ia explosion models. However, for simplicity in this work, we choose to anchor the relation with the well-studied SN Ia SN 2011fe. Modelling of the photospheric phase light curve for SN 2011fe by Pereira et al. (2013) yielded a ${ }^{56} \mathrm{Ni}$ mass of $M_{N i}=0.53 \pm 0.11 M_{\odot} . \mathrm{Re}-$ cently Mazzali et al. (2015) extended their spectroscopic modelling of the SN 2011fe spectral time series (presented for photospheric epochs in Mazzali et al. 2014) to nebular phase epochs and found $M_{N i}=0.47 \pm 0.08 M_{\odot}$. For simplicity in this work, we thus will choose a ${ }^{56} \mathrm{Ni}$ mass anchor for SN 2011fe of $M_{N i}=0.50 M_{\odot}$, yielding final ${ }^{56} \mathrm{Ni}$ mass values derived as:

$$
M_{N i}=0.50 M_{\odot} \frac{L_{C o}}{L_{11 f e}}
$$

where $\log \left(L_{11 f e}\right)=39.410$ is the scaled [Co III] $\lambda 5893$ luminosity we measure for SN $2011 \mathrm{fe}$ - this is used as a zeropoint for the remainder of our SN sample. The values for $M_{N i}$ for our sample are presented in Table 3 . In Section 6.3 we further discuss the implications of our ${ }^{56} \mathrm{Ni}$ mass values and their relation to the ejected masses of our SN Ia sample.

Other techniques have been presented for measuring the mass of ${ }^{56} \mathrm{Ni}$ produced in the SN Ia explosion. Stritzinger et al. (2006a) employed semi-empirical modelling of SN Ia bolometric light curves to measure the ejected mass and ${ }^{56} \mathrm{Ni}$ mass for a sample of 17 nearby SNe Ia. They then found that ${ }^{56} \mathrm{Ni}$ masses derived from modelling of the nebular spectra (Mazzali et al. 1997, 1998; Stehle et al. 2005) yielded consistent results (Stritzinger et al. 2006b). Seven of the SNe Ia from their sample are included in ours, and we show a comparison of our ${ }^{56} \mathrm{Ni}$ values versus those derived from their two methods in Figure 8 In some of the cases, our ${ }^{56} \mathrm{Ni}$ masses are somewhat lower than theirs (both for the light curve and nebular ${ }^{56} \mathrm{Ni}$ mass estimates) though generally show acceptable agreement. We note that for SN 1994ae and SN 2002er , Stritzinger et al. (2006b) employ a much higher reddening value than ours $(E(B-V)=0.15$ mag versus $E(B-V)=0.00 \mathrm{mag}$ for SN 1994ae and $E(B-V)=0.36$ mag versus $E(B-V)=$ $0.12 \mathrm{mag}$ for SN 2002er), which is likely the source of the discrepancy between our values.

\section{DISCUSSION}

In this Section we discuss the important physical implications of our observational results above. First, we examine the fact that the [Co III] $\lambda 5893$ line flux evolution requires a constant scaling between energy released by ${ }^{56} \mathrm{Co}$ decay and that absorbed by the nebula - this requires efficient local deposition of energy from positrons and near-complete escape of gamma-rays from the IGE core (Section 6.1). Next, we argue that the [Co III] $\lambda 5893$ evolution requires stable ionization conditions in the nebula for a period
Table 3. Final SN Ia Nickel Masses

\begin{tabular}{lll}
\hline SN & $\begin{array}{l}M_{N i} \\
\left(M_{\odot}\right)^{a}\end{array}$ & $\begin{array}{l}M_{e j} \\
\left(M_{\odot}\right)^{b}\end{array}$ \\
\hline SN1990N & $0.514 \pm 0.027(0.081)$ & $1.437 \pm 0.009$ \\
SN1991T & $1.049 \pm 0.106(0.308)$ & $1.407 \pm 0.019$ \\
SN1994ae & $0.458 \pm 0.013(0.069)$ & $1.417 \pm 0.013$ \\
SN1995D & $0.593 \pm 0.059(0.165)$ & $1.448 \pm 0.009$ \\
SN1998aq & $0.707 \pm 0.042(0.127)$ & $1.304 \pm 0.015$ \\
SN1998bu & $0.686 \pm 0.029(0.292)$ & $1.299 \pm 0.027$ \\
SN1999aa & $1.593 \pm 0.114(0.238)$ & $1.465 \pm 0.003$ \\
SN2002cs & $0.775 \pm 0.081(0.130)$ & $1.361 \pm 0.016$ \\
SN2002dj & $0.882 \pm 0.051(0.176)$ & $1.299 \pm 0.019$ \\
SN2002er & $0.344 \pm 0.018(0.082)$ & $1.202 \pm 0.015$ \\
SN2002fk & $0.625 \pm 0.016(0.120)$ & $1.346 \pm 0.016$ \\
SN2003du & $0.414 \pm 0.022(0.177)$ & $1.373 \pm 0.010$ \\
SN2003hv & $0.186 \pm 0.003(0.073)$ & $0.914 \pm 0.037$ \\
SN2004bv & $1.294 \pm 0.040(0.266)$ & $1.468 \pm 0.003$ \\
SN2004eo & $0.332 \pm 0.011(0.046)$ & $1.135 \pm 0.016$ \\
SN2005cf & $0.625 \pm 0.044(0.184)$ & $1.308 \pm 0.013$ \\
SN2007af & $0.440 \pm 0.029(0.071)$ & $1.289 \pm 0.017$ \\
SN2007gi & $0.624 \pm 0.027(0.248)$ & $1.149 \pm 0.023$ \\
SN2007le & $0.549 \pm 0.033(0.202)$ & $1.387 \pm 0.017$ \\
SN2007sr & $0.609 \pm 0.027(0.107)$ & $1.311 \pm 0.045$ \\
SN2009le & $0.673 \pm 0.065(0.102)$ & $1.380 \pm 0.026$ \\
SN2011by & $0.582 \pm 0.082(0.119)$ & $1.295 \pm 0.029$ \\
SN2011fe & $0.500 \pm 0.026(0.069)$ & $1.310 \pm 0.015$ \\
SN2011iv & $0.349 \pm 0.046(0.122)$ & $0.818 \pm 0.032$ \\
SN2012cg & $0.479 \pm 0.048(0.309)$ & $1.422 \pm 0.010$ \\
SN2012fr & $0.670 \pm 0.043(0.287)$ & $1.454 \pm 0.004$ \\
SN2012hr & $0.328 \pm 0.008(0.084)$ & $1.375 \pm 0.025$ \\
SN2013aa & $1.658 \pm 0.091(0.717)$ & $1.468 \pm 0.004$ \\
SN2013cs & $0.757 \pm 0.094(0.174)$ & $1.360 \pm 0.013$ \\
SN2013dy & $0.608 \pm 0.047(0.137)$ & $1.450 \pm 0.004$ \\
SN2013gy & $0.950 \pm 0.075(0.159)$ & $1.278 \pm 0.012$ \\
SN2014J & $0.837 \pm 0.176(0.250)$ & $1.441 \pm 0.007$ \\
\hline & & \\
&
\end{tabular}

${ }^{a}$ Nominal uncertainties arise from measurement errors in the Co line flux or SN reddening, while distance uncertainties are listed in parenthesis. Systematic error for $M_{N i}$ is estimated at $0.2 M_{\odot}$.

${ }^{b}$ Includes only measurement uncertaintes from SN light curve stretch. Systematic error for $M_{e j}$ is estimated at $0.1 M_{\odot}$.

of several hundred days, which we support by demonstrating stability of ionization-dependent flux ratios measured from the data (Section 6.2). Finally, we discuss potential interpretations of SN Ia explosion conditions implied by our observed relationship between inferred ${ }^{56} \mathrm{Ni}$ mass and ejected mass (Section 6.3).

\subsection{Gamma-ray transparency timescales for nebular SNe Ia}

For ${ }^{56}$ Co to deposit a constant fraction of its decay energy into the nebula, positrons from the decay must be efficiently trapped in the IGE core and gamma-rays must be able to effectively escape ${ }^{3}$. As noted above, efficient local positron energy deposition is expected to hold for the temperatures and densities encountered at these nebular phases (Axelrod 1980; Chan \& Lingenfelter 1993; Ruiz-Lapuente \& Spruit 1998). In practice, gamma-rays become

\footnotetext{
3 We do note that other physical properties of the nebula (e.g. ionization or emission measure changes) could somehow conspire to compensate for gamma-ray opacity to make the line emission evolve as $M_{C o}^{2}$, but we consider the gamma-ray transparency scenario to be the simplest explanation.
} 

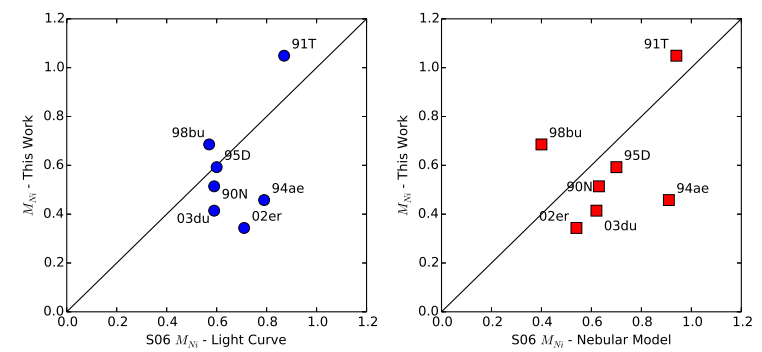

Figure 8. Comparison of our ${ }^{56} \mathrm{Ni}$ mass values to those derived by Stritzinger et al. (2006b) using light curve modelling (left) and nebular spectra modelling (right).

negligible after the time when the gamma-ray energy deposition equals that from positrons, which occurs when the optical depth drops enough to reach this equality (Section 4.1). We will refer to this henceforth as the "transparency" timescale $t_{c}$-after this epoch positrons dominate energy deposition in the nebula.

We fit the transparency timescale for several supernovae in Section 4.3 and found several have longer transparency times $\left(t_{c} \sim 180\right.$ days) close to the theoretical expectation for the DDC10 model. Previous analysis of gamma-ray transparency timescales found similar results: $t_{c} \approx 170$ days for SN $2000 \mathrm{cx}$ (Sollerman et al. 2004) and $t_{c} \approx 188$ days for SN 2001el (Stritzinger \& Sollerman 2007). However we found that other SNe Ia (notably SN 2011fe and SN 2007af) had much shorter transparency times $\left(t_{c} \sim 80\right.$ days). This variation in transparency times may reflect a diversity in nebular densities, as most of the gamma-ray opacity at these late epochs will come from opacity from electrons in the nebula. Interestingly, the SNe Ia with shorter transparency times (SN 2011fe and SN 2007af) have lower stretch values than most of the $\mathrm{SNe}$ Ia with longer transparency times (SN 2003du, SN 2012fr, SN 2013aa), possibly indicating some relationship between nebular density and stretch. The one exception to this is SN 2003hv, which appears to have low stretch but long transparency time (and thus would imply high density) - this result is opposite to the findings of Mazzali et al. (2011) who found SN 2003hv had reduced density in the inner regions of the ejecta. The source of this discrepancy is unclear, but may constitute further evidence that SN 2003hv is a "non-standard" event.

Because of the diversity in gamma-ray transparency timescales in the SNe Ia we tested, it is likely that the impact of gamma-ray energy deposition on the [Co III] $\lambda 5893$ flux will be impacted by similar variability. Given the results above (Section 4.3 ) this may result in an average uncertainty of $30 \%$ on the final ${ }^{56} \mathrm{Ni}$ masses we infer. The only robust way to account for gamma-ray opacity effects is to obtain a nebular time series. However the transparency time is best constrained by observations from 100-150 days when the $\mathrm{SN}$ is only 3-4 magnitudes fainter than peak. Thus it should be observationally feasible to obtain such data for future $\mathrm{SNe}$ Ia observed in the nebular phase.

More interestingly, the time evolution of the [Co III] $\lambda 5893$ flux presents a new method for measuring the gamma-ray transparency time scale, as it gives a direct probe of the energy being deposited into the nebula. Previously this could only be done with the aid of bolometric light curves (Sollerman et al. 2004; Stritzinger \& Sollerman 2007; Leloudas et al. 2009), which necessarily rely on extensive optical and infrared photometry and/or uncertain bolometric corrections. Instead, our method requires only two nebular spectra with contemporaneous optical photometry.
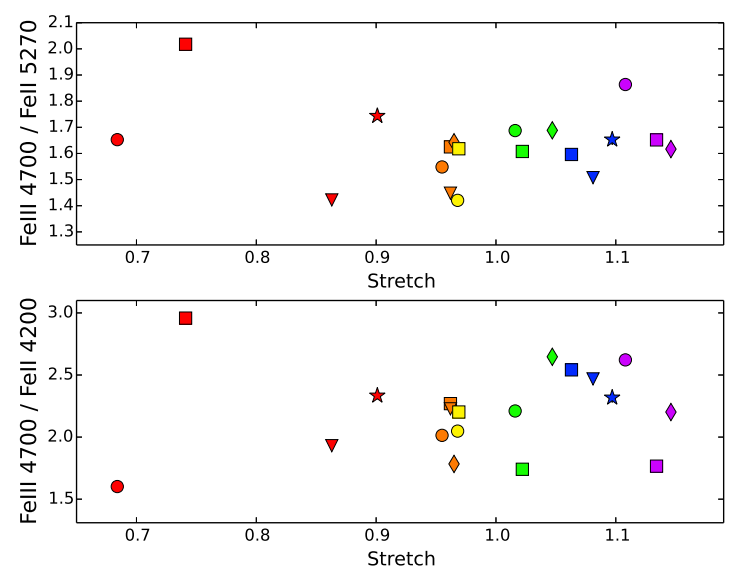

Figure 10. Integrated flux ratios of the Fe III $4700 \AA$ complex compared to the Fe II $5270 \AA$ complex (top) and Fe II $4200 \AA$ complex (bottom) as a function of light curve stretch (SiFTO $s$ ) for all SNe Ia in our sample. Formal spectrum flux error bars are smaller than the data markers. Markers are the same as for Figure 7

\subsection{Ionization conditions in the $\mathrm{SN}$ nebula}

As noted above, the consistency of the [Co III] $\lambda 5893$ flux evolution with the square of the cobalt mass implies a constant scaling between the energy being absorbed by cobalt atoms and the energy they emit in the [Co III] $\lambda 5893$ line. This implies stability in the ionization conditions of the nebula, which we now investigate from a more detailed inspection of our nebular spectra.

To confirm that the ionization state of the nebula is indeed slowly evolving from phases $150 \leq t \leq 400$ days, we examine the flux ratios of nebular emission lines arising primarily from Fe II and Fe III. If the ratio of these lines evolves with time, this would indicate a change in the ionization state. In the left panels of Figure 9 we highlight the regions of the typical SN Ia nebular spectra (here from SN 2011fe and SN 2012fr) which are dominated by strong line complexes of either Fe II or Fe III. We integrate the flux in these regions for all the nebular SN Ia spectra in our sample, and in the right panels of Figure 9 we show how the line flux ratios evolve with phase for the nebular time series SNe Ia (the same as from Section 4). For this analysis we only consider phases later than $t \sim 200$ days, as this is when this region of the spectrum is reliably optically thin (see Section 4) - note this cuts SN 2007af from the Fe time series sample.

Though there is indeed some evolution in the flux ratio of Fe II lines to Fe III lines, it is comparatively small - generally less than $10 \%$ change of the relative line flux in Fe III compared to Fe II. In sharp contrast, consider the Fe III/Fe II line flux ratios as measured from the $t \sim 1000$ days spectrum for SN 2011fe from Taubenberger et al. (2015) -0.52 for $4700 / 5270$ versus a mean of 1.6 at earlier phases, and 0.87 for $4700 / 4200$ versus an earlier mean of 2.3 - which decrease by at least $65 \%$ from their values in the $150 \leq t \leq 400$ day range [we note these values should be considered upper limits as it appears that the Fe III 4700 line has effectively disappeared in the $t \sim 1000$ days spectrum for SN 2011fe, so the flux we measure here is likely due to other species]. By these very late phases the physical conditions in the SN Ia nebula have clearly changed in a dramatic fashion. Such is not the case for the SNe Ia in our sample at phases $150 \leq t \leq 400$ days.

In order to meaningfully compare the [Co III] $\lambda 5893$ line flux 

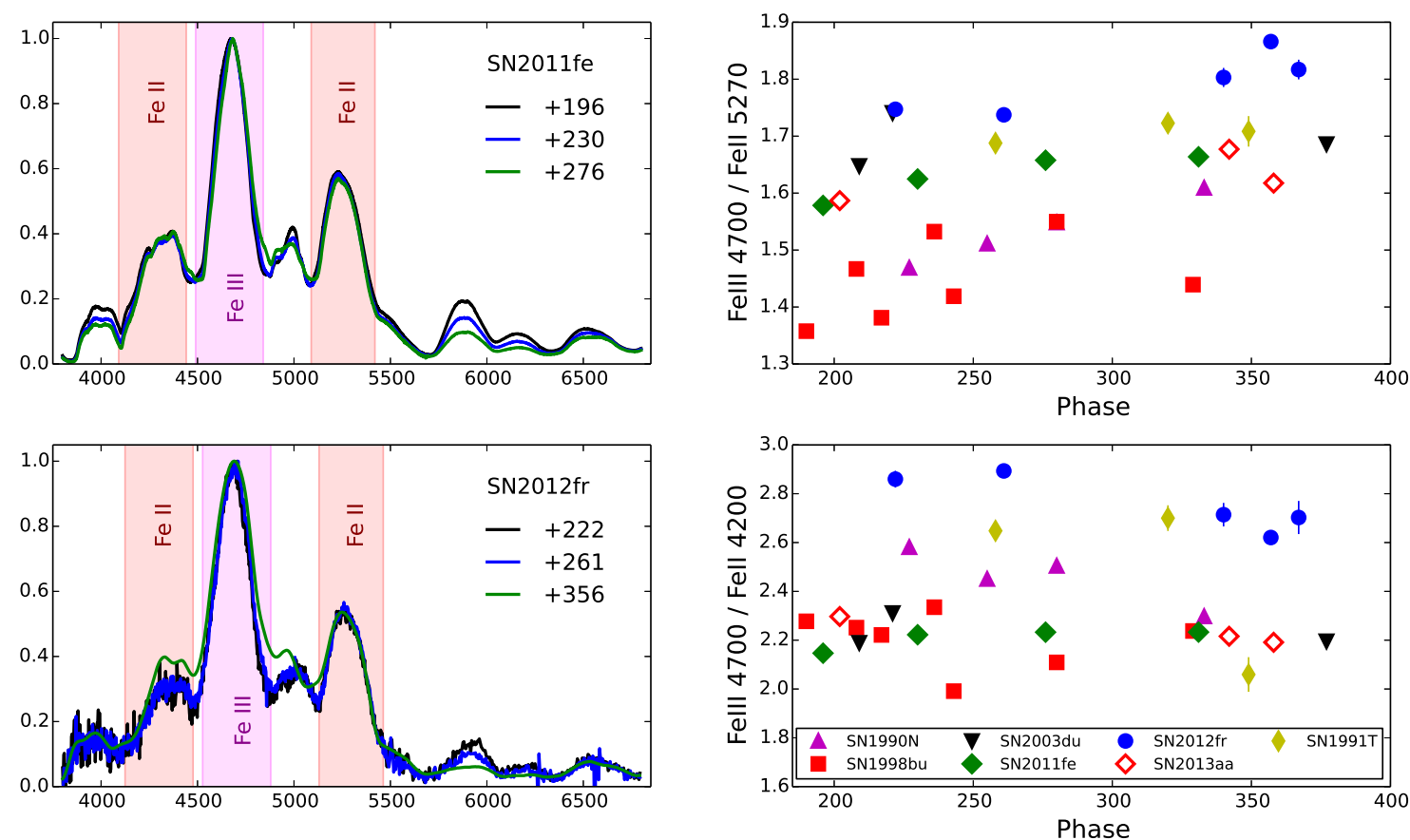

Figure 9. Left panels: Multiple nebular phase spectra of SN 2011fe (top) and SN 2012fr (bottom), highlighting the flux integration regions for the line complexes dominated by Fe II (light red regions) and Fe III (light magenta regions). Integration zones are shifted by the central redshift of the nebular Fe lines, here a blueshift of $\sim 600 \mathrm{~km} \mathrm{~s}^{-1}$ for SN $2011 \mathrm{fe}$ and a redshift of $\sim 1800 \mathrm{~km} \mathrm{~s}^{-1}$ for SN $2012 \mathrm{fr}$. Right panels: Temporal evolution of the ratios of the flux integral for the Fe III $4700 \AA \AA$ complex compared to the Fe II $5270 \AA \AA$ complex (top) and Fe II $4200 \AA$ complex (bottom) for SNe Ia with nebular time series.

from different SNe Ia, another key requirement is that the ionization state of all SNe Ia be relatively similar. To test this assumption, we again use the Fe line ratios described above, but plot the mean Fe III/Fe II line flux ratio (computed as the error-weighted mean for SNe Ia with multiple epochs) versus light curve stretch in Figure 10 We have excluded the highly reddened SN 2007le and SN 2014J to avoid any biases in these ratios due to uncertainty in the dust law (i.e. $R_{V}$ ).

Here we see some mild coherent change in the Fe line flux ratios (and thus ionization state) as a function of light curve stretch (with SN 2003hv as an outlier, as previously noted by Mazzali et al. 2011). Here the overall range of the line ratios is somewhat larger, with variations perhaps up to $40 \%$ but with a scatter of $7 \%$ (for $4700 / 5270$ ) and $15 \%$ (for 4700/4200). The ionization potentials of $\mathrm{Fe}$ and $\mathrm{Co}$ are very similar, which means a change in Fe III line flux induced by variation of the ionization state will manifest a comparable change in Co III line flux. Thus our [Co III] $\lambda 5893$ line fluxes above should have an additional scatter due to ionization state variations of about $10 \%$. Since our inferred ${ }^{56} \mathrm{Ni}$ masses are proportional to this line flux, this means that ionization state variations could induce a scatter of similar magnitude in our ${ }^{56} \mathrm{Ni}$ masses.

Our measurement of the [Co III] $\lambda 5893$ line flux evolution, and variations of $\mathrm{Fe}$ III/Fe II line flux ratios as a function of both phase and $\mathrm{SN}$ stretch, coherently indicate that the ionization states of normal SNe Ia are remarkably consistent across different $\mathrm{SNe}$ and nearly constant across phases $150 \leq t \leq 400$ days. This stability of the ionization state was predicted by Axelrod (1980), and our results here present the most compelling evidence to-date in support of that prediction.

\subsection{The relationship between ${ }^{56} \mathrm{Ni}$ and Ejected Mass}

The relationship between [Co III] $\lambda 5893$ luminosity and light curve stretch (Figure 7 hints at a relationship between physical properties of the SN Ia progenitor system. In Section 5.2 we converted our measured [Co III] $\lambda 5893$ line luminosities into inferred ${ }^{56} \mathrm{Ni}$ masses. Here we convert light curve stretch into the SN ejected mass (i.e. progenitor mass for $\mathrm{SNe}$ Ia) using the relationship between $M_{e j}$ and light curve stretch discovered by Scalzo et al. (2014a). Scalzo et al. (2014b) used Bayesian inference to model the intrinsic distribution of ejected masses, which can be folded in as an additional prior when determining ejected mass using this relation. We derive a cubic fit to the relationship between stretch and $M_{e j}$ :

$$
\begin{aligned}
M_{e j} & =2.07-7.51 s+11.56 s^{2}-4.77 s^{3} \\
& =1.35+1.30(s-1)-2.75(s-1)^{2}-4.77(s-1)^{3}
\end{aligned}
$$

The resultant values for ejected mass $\left(M_{e j}\right)$ we derive are presented in Table 3 along with our ${ }^{56} \mathrm{Ni}$ masses.

In Figure 11 we plot our inferred ${ }^{56} \mathrm{Ni}$ masses against these ejected masses. We note that there is a systematic uncertainty associated with $M_{e j}$ calculation of about $0.1 M_{\odot}$, as determined by Scalzo et al. (2014a) from recovering masses of SN Ia explosion models. For ${ }^{56} \mathrm{Ni}$ masses, we previously noted several sources of uncertainty: $10 \%$ uncertainty in the [Co III] $\lambda 5893$ flux itself (Section 3.2), 5\% uncertainty on the $t=200$ [Co III] $\lambda 5893$ luminosity due to uncertainty in the explosion date (Section 5.1, $10 \%$ from ionization state variations (Section 6.2), and possibly $30 \%$ from variations in gamma-ray transparency timescales (Section 6.1). Collectively this constitutes a possible $35 \%$ uncertainty 


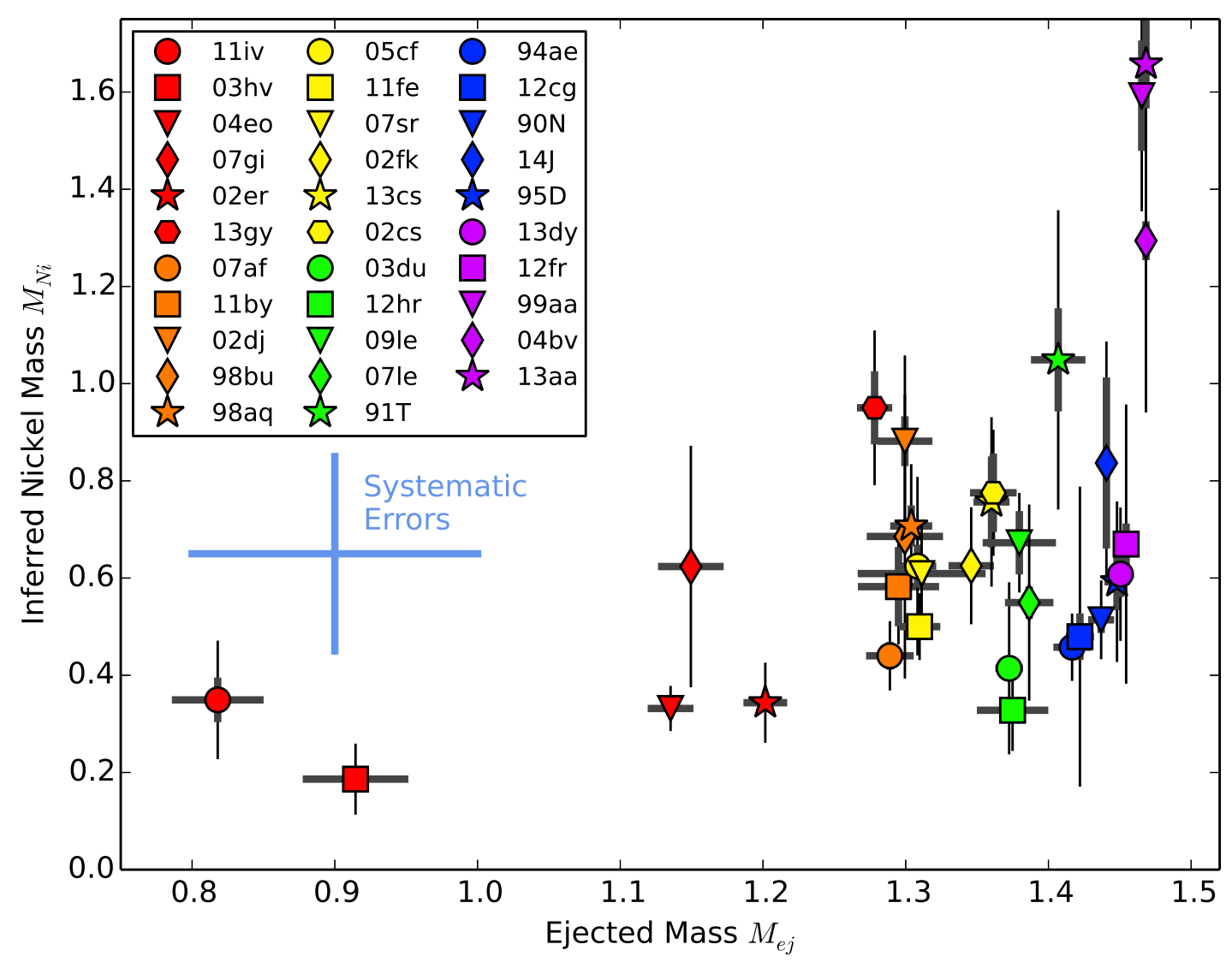

Figure 11. Mass of ${ }^{56} \mathrm{Ni}$ inferred from the scaled [Co III] $\lambda 5893$ line luminosity versus total ejected mass (i.e., progenitor WD mass) inferred from SN light curve stretch. As in Figure 6 thick error bars correspond to flux measurement errors (including colour correction uncertainties), while narrow error bars corresponding to distance uncertainties arising from peculiar velocities. The typical systematic uncertainties for estimating $M_{e j}\left( \pm 0.1 M_{\odot}\right.$ - from the stretch$M_{e j}$ relation of Scalzo et al. 2014b) and $M_{N i}\left( \pm 0.2 M_{\odot}\right.$ - from gamma-rays, explosion date uncertainty, and possible line contamination) are shown as the blue error bars in the left side of the plot. Note the anomalously high $M_{N i}$ values for SN 1999aa and SN 2013aa, which we attribute to line contamination and distance uncertainty, respectively (see text for details).

in our ${ }^{56} \mathrm{Ni}$ masses, which given the values we find would produce a mean uncertinty in $M_{N i}$ of about $0.2 M_{\odot}$.

The relation between $M_{N i}$ and $M_{e j}$ shows potential evidence for two regimes for the production of ${ }^{56} \mathrm{Ni}$ in $\mathrm{SNe}$ Ia. For subChandrasekhar ejected masses $\left(M_{e j} \lesssim 1.3 M_{\odot}-\right.$ though note SN 1991bg-like objects are not included in this analysis), the amount of ${ }^{56} \mathrm{Ni}$ produced is clustered around $M_{N i} \sim 0.4 M_{\odot}$, with a possible increase of $M_{N i}$ with $M_{e j}$ (though we note the statistics are small). Chandrasekhar-mass progenitors $\left(M_{e j} \approx 1.4 \pm 0.1 M_{\odot}\right)$ produce ${ }^{56} \mathrm{Ni}$ masses ranging from $0.4 M_{\odot} \lesssim M_{N i} \lesssim 1.2 M_{\odot}$, with the extreme high ${ }^{56} \mathrm{Ni}$ masses $\left(M_{N i} \gtrsim 1.0 M_{\odot}\right)$ occuring in SNe Ia spectroscopically similar to the peculiar SNe SN 1991T (SN 1999aa, SN 2004bv, SN 2013aa, and SN 1991T itself). Recently, Fisher \& Jumper (2015) suggested that Chandrasekharmass SN Ia progenitors preferentially lack a vigorous deflagration phase following the initial ignition, and result in a nearly pure detonation that produces about $1.0 M_{\odot}$ of ${ }^{56} \mathrm{Ni}$ and shows similarity to SN 1991T. Our findings that the [Co III] $\lambda 5893$ luminosity is exceptionally high only in 91T-like SNe Ia could lend support to this theory.
We note that SN 1999aa and SN 2013aa have anomalously high $M_{N i}$ values (indeed exceeding their $M_{e j}$ values). We visually inspected the spectra of these $\mathrm{SNe}$, and find no fault in our fits to the [Co III] $\lambda 5893$ line. SN 1999aa notably has the broadest linewidth of our sample, which could result in contamination of our measured [Co III] $\lambda 5893$ flux by nearby Fe II lines (see Figure2). SN 2013aa has a relatively uncertain distance to its host galaxy. We expect the true $M_{N i}$ for these two SNe is likely to be closer to that of the other SN 1991T-like SNe Ia, near $1.0--1.2 M_{\odot}$.

To compare model predictions with our inferred ${ }^{56} \mathrm{Ni}$ mass values, we gather ejected mass and ${ }^{56} \mathrm{Ni}$ mass outcomes from numerous SN Ia explosion models and plot them against our data in Figure 12 These models can be generally grouped into three categories: sub-Chandrasekhar mass detonations, Chandrasekhar-mass deflagration to detonation transitions (DDT), and Chandrasekharmass deflagrations which fail to detonate. We discuss each category and its agreement with the data below.

Sub-Chandrasekhar (sub-Ch) mass detonations: We consider sub-Ch detonations from Sim et al. (2010), where detonations were artificially initiated in WDs of varying initial masses. These 


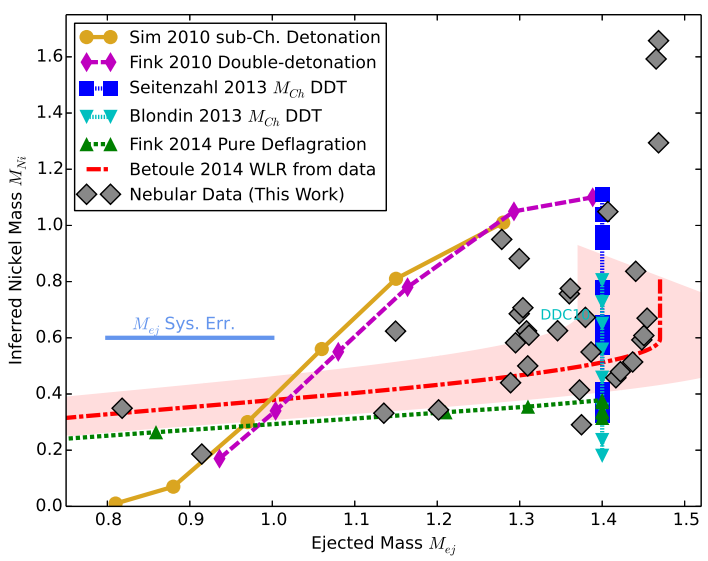

Figure 12. $M_{N i}$ and $M_{e j}$ values inferred from data (dark grey diamonds data as in Figure 11 compared to various theoretical models: pure detonation models from sub-Chandra detonations (Sim et al. 2010, yellow circles) and double-detonations (Fink et al. 2010, magenta diamonds); detonationdeflagration transitions (DDT) from Seitenzahl et al. (2013b, blue sqaures) and Blondin et al. (2013, cyan downward triangles); and Chadra-mass pure deflagrations (Fink et al. 2014, green upward triangls). The observed widthluminosity relation (and its scatter) from the recent cosmology analysis of Betoule et al. (2014) are shown as the red curve (and light red shaded area).

models are also applicable to sub-Ch WDs ignited via other mechanisms (e.g. a violent merger), and were also employed to estimate the brightness distribution of violent mergers in Ruiter et al. (2013). We also examine sub-Ch double detonation models from Fink et al. (2010): these are qualitatively similar to the Sim et al. (2010) but the ignition mechanism naturally arises from a surface helium layer ignition. Both models show a similar relationship between $M_{N i}$ and $M_{e j}$, which shows a much steeper increase of $M_{N i}$ with $M_{e j}$ than we infer from our data. However we note that with the systematic uncertainty in $M_{e j}$ estimates (from stretch) these may be compatible with the data.

Deflagration to detonation transitions (DDT): We present models from both Seitenzahl et al. (2013b) and Blondin et al. (2013) - including the DDC10 model employed for radiative transfer calculations in Section 3.1. In general for these models the $M_{C h}$ progenitors undergo an initial deflagration phase which transitions to a detonation at a later time: the timing of this transition directly sets the amount of ${ }^{56} \mathrm{Ni}$ produced. For Seitenzahl et al. (2013b), the DDT time was calculated from the sub-grid scale turbulent energy (Ciaraldi-Schoolmann et al.2013) which in practice varied with the vigorousness of the initial deflagration (set by hand as the number of initial ignition points). For Blondin et al. (2013), the DDT time is set by a manual trigger. Both sets of models cover a range of ${ }^{56} \mathrm{Ni}$ mass production, similar to the range inferred from our data.

Pure deflagrations: Finally we consider pure deflagration models presented in Fink et al. (2014). These models are variations on the Chandrasekhar-mass Seitenzahl et al. (2013b) models in which the DDT module has been intentionally turned off. Many of these deflagration models fail to fully unbind the star and eject only a portion of the WD's total mass and leave a bound remnant we note that the Scalzo et al. (2014b) method for estimating ejected mass from light curves is not trained to account for bound remants so may have some additional systematic uncertainty for this explosion mechanism. Interestingly, these models show a weak depen- dence of $M_{N i}$ on $M_{e j}$ for sub-Ch ejected masses, similar to what we infer for this regime of the data. This also shows some agreement with the width-luminosity relation observed in cosmological supernova samples - we show this as well in Figure 12 using the WLR from Betoule et al. (2014) converted to $M_{N i}$ and $M_{e j}$ using the relations presented in Scalzo et al. (2014b).

If indeed the $M_{N i}-M_{e j}$ trend arises from two distinct explosion mechanisms for SNe Ia, several key questions remain to be answered with future research. One such question is where the split between the two mechanisms occurs - SNe Ia at $M_{C h}$ with $\sim 0.5 M_{\odot}$ of ${ }^{56} \mathrm{Ni}$ could arise from either mechanism - and what physical property of the progenitor decides which mechanism occurs. Next we should investigate why the two mechanisms produce SNe Ia which obey the same width-luminosity relation - one might expect that a different relationship between $M_{N i}$ and $M_{e j}$ would yield different relationship between peak luminosity and light curve width. Such insights could be further advanced by study of other related thermonuclear explosions which span a broader range of $M_{N i}$ and $M_{e j}$ (e.g., McCully et al. 2014b, see their Figure 15).

Finally, we most critically should assess whether the two mechanisms calibrate cosmological distances in the same fashion. Recent evidence has been mounting that $\mathrm{SNe}$ Ia show progenitor signatures (e.g. CSM interaction, high-velocity features, host galaxy properties) which appear to clump into two groups (Maguire et al. 2014). In parallel, SN Ia cosmological analyses have found that SNe Ia in high- and low-mass galaxies have subtly different standardized luminosities (Sullivan et al. 2010; Kelly et al. 2010; Lampeitl et al. 2010; Gupta et al. 2011; D'Andrea et al. 2011; Konishi et al. 2011; Galbany et al. 2012; Hayden et al. 2013; Johansson et al. 2013; Childress et al. 2013b; Rigault et al.2013; Childress et al. 2014c; Kelly et al. 2015). These and the current study motivate further examination of the environments and standardized luminosities of SNe Ia whose ${ }^{56} \mathrm{Ni}$ mass and ejected mass are assessed with the techniques presented here. Such a study is limited by distance uncertainties, and thus should be targeted at SNe Ia in the nearby smooth Hubble flow $(z \geq 0.015)$ where distance uncertainties from peculiar velocities become small $(\leq 0.10 \mathrm{mag})$.

\section{CONCLUSIONS}

In this work we examine the [Co III] $\lambda 5893$ feature in 94 nebular phase (150 $\leq t \leq 400$ days past peak brightness) spectra of 32 SNe Ia compiled from the literature and new observations. This feature arises predominantly from radioactive ${ }^{56} \mathrm{Co}$, the decay product of ${ }^{56} \mathrm{Ni}$ (which powers the bright early light curve) - thus this feature provides a direct window for investigating the power source behind SN Ia light curves.

We used nebular time series for eight SNe Ia to show that the temporal evolution of the [Co III] $\lambda 5893$ flux falls very close to the square of the mass of ${ }^{56} \mathrm{Co}$ as a function of time. This is the expected dependence in the limit where the nebula is fully optically thin to gamma-rays produced in the ${ }^{56} \mathrm{Co}$ decay but locally thermalizes energy from positrons emitted in the decay. We then used this uniform time dependence to infer the relative amount of ${ }^{56} \mathrm{Ni}$ produced by all 32 SNe Ia in our sample by using SN $2011 \mathrm{fe}$ as an anchor (at $M_{N i}=0.5 M_{\odot}$ ).

The greatest systematic uncertainty in our ${ }^{56} \mathrm{Ni}$ mass measurements was the time at which the nebula becomes effectively optically thin to gamma-rays (which we define by the "crossing" time when energy deposition from positrons begins to exceed that of 
gamma-rays). Though this could intoduce $30 \%$ uncertainty in ${ }^{56} \mathrm{Ni}$ masses (on average, though this is time dependent), we showed that the gamma-ray transparency time can be readily measured when multiple nebular spectra are available. In particular, a single spectrum at phases $100 \leq t \leq 150$ days past maximum light - when the SN is only 3-4 magnitudes fainter than peak - can easily constrain the gamma-ray transparency time. This can robustify our technique for measuring ${ }^{56} \mathrm{Ni}$ masses of future $\mathrm{SNe}$ Ia, but the gamma-ray transparency time itself could provide important clues to $\mathrm{SN}$ Ia progenitor properties.

When comparing our inferred ${ }^{56} \mathrm{Ni}$ masses to the ejected masses of our SN Ia sample (using techniques from Scalzo et al. $2014 \mathrm{a}$ b), we find evidence for two regimes in the production of ${ }^{56} \mathrm{Ni}$ (which are too distinct to be an artefact of systematic uncertainties). For low ejected masses (low stretch), $M_{N i}$ clusters at low values $\left(M_{N i} \approx 0.4 M_{\odot}\right)$. At high ejected masses (high stretch) near the Chandrasekhar mass, $M_{N i}$ has a much larger spread $\left(0.4 M_{\odot} \lesssim M_{N i} \lesssim 1.2 M_{\odot}\right)$. This could constitute evidence for two distinct explosion mechanisms in SNe Ia.

This work has illustrated the power of the nebular [Co III] $\lambda 5893$ feature in probing the fundamental explosion physics of $\mathrm{SNe}$ Ia. We provide a simple recipe for calculating ${ }^{56} \mathrm{Ni}$ mass from the [Co III] $\lambda 5893$ line flux from a single nebular epoch, as well as prescription for a more robust measurement that accounts for opacity effects by using multiple nebular epochs. Future measurements which eliminate the opacity systematics and distance uncertainties could provide a detailed understanding of the explosion mechanisms for SNe Ia.

Acknowledgements: This research was conducted by the Australian Research Council Centre of Excellence for All-sky Astrophysics (CAASTRO), through project number CE110001020. BPS acknowledges support from the Australian Research Council Laureate Fellowship Grant FL0992131. ST acknowledges support by TRR33 "The Dark Universe" of the German Research Foundation (DFG). DJH acknowledges support from NASA theory grant NNX14AB41G. KM is supported by a Marie Curie Intra-European Fellowship, within the 7th European Community Framework Programme (FP7). NER acknowledges the support from the European Union Seventh Framework Programme (FP7/2007-2013) under grant agreement n. 267251 "Astronomy Fellowships in Italy (AstroFIt). MF acknowledges support from the European Union FP7 programme through ERC grant number 320360. MS acknowledges support from the Royal Society and EU/FP7-ERC grant $\mathrm{n}^{\circ}$ [615929]. AGY is supported by the EU/FP7 via ERC grant no. 307260, the Quantum Universe I-Core program by the Israeli Committee for planning and budgeting and the ISF; by Minerva and ISF grants; by the Weizmann-UK "making connections" program; and by Kimmel and ARCHES awards. AMG acknowledges financial support by the Spanish Ministerio de Economía y Competitividad (MINECO) grant ESP2013-41268-R. The research leading to these results has received funding from the European Union Seventh Framework Programme [FP7/2007-2013] under grant agreement num. 264895. SJS acknowledges funding from the European Research Council under the European Union's Seventh Framework Programme (FP7/2007-2013)/ERC Grant agreement $\mathrm{n}^{\mathrm{o}}$ [291222] and STFC grants ST/I001123/1 and ST/L000709/1.

Part of this research was conducted while John Hillier was a Distinguished Visitor at the Research School of Astronomy and Astrophysics at the Australian National University. This research is based on observations collected at the European Organisation for Astronomical Research in the Southern Hemisphere, Chile as part of PESSTO (the Public ESO Spectroscopic Survey for Transient Objects), ESO program ID 188.D-3003.

We thank Stefano Benetti and Massimo Della Valle for helpful comments. We also thank Ben Shappee for kindly providing spectra of SN $2011 \mathrm{fe}$.

Some of the data presented herein were obtained at the W.M. Keck Observatory, which is operated as a scientific partnership among the California Institute of Technology, the University of California and the National Aeronautics and Space Administration. The Observatory was made possible by the generous financial support of the W.M. Keck Foundation. The authors wish to recognize and acknowledge the very significant cultural role and reverence that the summit of Mauna Kea has always had within the indigenous Hawaiian community. We are most fortunate to have the opportunity to conduct observations from this mountain. This research has made use of the Keck Observatory Archive (KOA), which is operated by the W. M. Keck Observatory and the NASA Exoplanet Science Institute (NExScI), under contract with the National Aeronautics and Space Administration.

This research has made use of the NASA/IPAC Extragalactic Database (NED) which is operated by the Jet Propulsion Laboratory, California Institute of Technology, under contract with the National Aeronautics and Space Administration. This research has made use of NASA's Astrophysics Data System (ADS).

\section{REFERENCES}

Aldering, G., et al. 2006, ApJ, 650, 510

Altavilla, G., et al. 2004, MNRAS, 349, 1344

Anupama, G. C., Sahu, D. K., \& Jose, J. 2005, A\&A, 429, 667

Arnett, W. D. 1982, ApJ, 253, 785

Aubourg, É., Tojeiro, R., Jimenez, R., Heavens, A., Strauss, M. A., \& Spergel, D. N. 2008, A\&A, 492, 631

Axelrod, T. S. 1980, PhD thesis, California Univ., Santa Cruz.

Betoule, M., et al. 2014, A\&A, 568, A22

Blinnikov, S. I., \& Khokhlov, A. M. 1986, Soviet Astronomy Letters, 12, 131

Blondin, S., Dessart, L., \& Hillier, D. J. 2015, MNRAS, 448, 2766

Blondin, S., Dessart, L., Hillier, D. J., \& Khokhlov, A. M. 2013, MNRAS, 429, 2127

Blondin, S., et al. 2012, AJ, 143, 126

Bloom, J. S., et al. 2012, ApJL, 744, L17

Branch, D. 1992, ApJ, 392, 35

Branch, D., Fisher, A., \& Nugent, P. 1993, AJ, 106, 2383

Branch, D., et al. 2003, AJ, 126, 1489

Brown, T. M., et al. 2013, PASP, 125, 1031

Bureau, M., Mould, J. R., \& Staveley-Smith, L. 1996, ApJ, 463, 60

Buton, C., et al. 2013, A\&A, 549, A8

Cao, Y., et al. 2015, Nature, 521, 328

Cappellari, M., et al. 2011, MNRAS, 413, 813

Cappellaro, E., et al. 2001, ApJL, 549, L215

Cardelli, J. A., Clayton, G. C., \& Mathis, J. S. 1989, ApJ, 345, 245

Catinella, B., Haynes, M. P., \& Giovanelli, R. 2005, AJ, 130, 1037

Chan, K.-W., \& Lingenfelter, R. E. 1993, ApJ, 405, 614

Childress, M., et al. 2013a, ApJ, 770, 107

—. 2013b, ApJ, 770, 108

Childress, M. J., Filippenko, A. V., Ganeshalingam, M., \& Schmidt, B. P. 2014a, MNRAS, 437, 338 
Childress, M. J., Vogt, F. P. A., Nielsen, J., \& Sharp, R. G. 2014b, Ap\&SS, 349, 617

Childress, M. J., Wolf, C., \& Zahid, H. J. 2014c, MNRAS, 445, 1898

Childress, M. J., et al. 2013c, ApJ, 770, 29

Chiosi, E., Chiosi, C., Trevisan, P., Piovan, L., \& Orio, M. 2015, MNRAS, 448, 2100

Chotard, N., et al. 2011, A\&A, 529, L4+

Churazov, E., et al. 2014, Nature, 512, 406

Ciaraldi-Schoolmann, F., Seitenzahl, I. R., \& Röpke, F. K. 2013, A\&A, 559, A117

Colgate, S. A., \& McKee, C. 1969, ApJ, 157, 623

Conley, A., et al. 2008, ApJ, 681, 482

Contreras, C., et al. 2010, AJ, 139, 519

Cortés, J. R., Kenney, J. D. P., \& Hardy, E. 2008, ApJ, 683, 78

Crawford, S. M., et al. 2010, in Society of Photo-Optical Instrumentation Engineers (SPIE) Conference Series, Vol. 7737, Observatory Operations: Strategies, Processes, and Systems III. Edited by Silva, David R.; Peck, Alison B.; Soifer, B. Thomas.

Crotts, A. P. S., \& Yourdon, D. 2008, ApJ, 689, 1186

D'Andrea, C. B., et al. 2011, ApJ, 743, 172

de Vaucouleurs, G., de Vaucouleurs, A., Corwin, Jr., H. G., Buta, R. J., Paturel, G., \& Fouqué, P. 1991, Third Reference Catalogue of Bright Galaxies. Volume I: Explanations and references. Volume II: Data for galaxies between $0^{h}$ and $12^{h}$. Volume III: Data for galaxies between $12^{h}$ and $24^{h}$.

Dessart, L., Blondin, S., Hillier, D. J., \& Khokhlov, A. 2014a, MNRAS, 441, 532

Dessart, L., Hillier, D. J., Blondin, S., \& Khokhlov, A. 2014b, MNRAS, 439, 3114

-. 2014c, MNRAS, 441, 3249

Diehl, R., et al. 2014, Science, 345, 1162

Dilday, B., et al. 2012, Science, 337, 942

Dopita, M., Hart, J., McGregor, P., Oates, P., Bloxham, G., \& Jones, D. 2007, Ap\&SS, 310, 255

Dopita, M., et al. 2010, Ap\&SS, 327, 245

Faber, S. M., et al. 2003, in Society of Photo-Optical Instrumentation Engineers (SPIE) Conference Series, Vol. 4841, Instrument Design and Performance for Optical/Infrared Groundbased Telescopes, ed. M. Iye \& A. F. M. Moorwood, 1657-1669

Filippenko, A. V., et al. 1992a, ApJL, 384, L15

-. 1992b, AJ, 104, 1543

Fink, M., Röpke, F. K., Hillebrandt, W., Seitenzahl, I. R., Sim, S. A., \& Kromer, M. 2010, A\&A, 514, A53

Fink, M., et al. 2014, MNRAS, 438, 1762

Fisher, R., \& Jumper, K. 2015, arXiv:1504.00014

Foley, R. J., et al. 2012, ApJL, 753, L5

-. 2013, ApJ, 767, 57

-. 2014, MNRAS, 443, 2887

Förster, F., González-Gaitán, S., Folatelli, G., \& Morrell, N. 2013, ApJ, 772, 19

Galbany, L., et al. 2012, ApJ, 755, 125

Ganeshalingam, M., et al. 2010, ApJS, 190, 418

Gasques, L. R., Afanasjev, A. V., Aguilera, E. F., Beard, M., Chamon, L. C., Ring, P., Wiescher, M., \& Yakovlev, D. G. 2005, PhysRevC, 72, 025806

Gómez, G., \& López, R. 1998, AJ, 115, 1096

Graham, A. W., Colless, M. M., Busarello, G., Zaggia, S., \& Longo, G. 1998, A\&AS, 133, 325

Graham, M. L., et al. 2015, MNRAS, 446, 2073

Graur, O., Zurek, D., Shara, M. M., \& Riess, A. G. 2015, arXiv: 1505.00777
Guillochon, J., Dan, M., Ramirez-Ruiz, E., \& Rosswog, S. 2010, ApJL, 709, L64

Gupta, R. R., et al. 2011, ApJ, 740, 92

Guthrie, B. N. G., \& Napier, W. M. 1996, A\&A, 310, 353

Guy, J., et al. 2007, A\&A, 466, 11

-. 2010, A\&A, 523, A7+

Hamuy, M., et al. 2003, Nature, 424, 651

Hansen, J. E., Raassen, A. J. J., \& Uylings, P. H. M. 1984, ApJ, 277, 435

Hayden, B. T., Gupta, R. R., Garnavich, P. M., Mannucci, F., Nichol, R. C., \& Sako, M. 2013, ApJ, 764, 191

Hicken, M., et al. 2009, ApJ, 700, 331

-. 2012, ApJS, 200, 12

Hillier, D. J., \& Dessart, L. 2012, MNRAS, 424, 252

Ho, W. C. G., Van Dyk, S. D., Peng, C. Y., Filippenko, A. V., Leonard, D. C., Matheson, T., Treffers, R. R., \& Richmond, M. W. 2001, PASP, 113, 1349

Hoeflich, P., \& Khokhlov, A. 1996, ApJ, 457, 500

Hook, I. M., Jørgensen, I., Allington-Smith, J. R., Davies, R. L., Metcalfe, N., Murowinski, R. G., \& Crampton, D. 2004, PASP, 116,425

Howell, D. A., et al. 2006, Nature, 443, 308

-. 2009, ApJ, 691, 661

Hoyle, F., \& Fowler, W. A. 1960, ApJ, 132, 565

Iben, Jr., I., \& Tutukov, A. V. 1984, ApJS, 54, 335

-. 1991, ApJ, 370, 615

Jeffery, D. J. 1999, astro-ph/9907015

Jha, S., et al. 1999, ApJS, 125, 73

-. 2006, AJ, 131, 527

Johansson, J., et al. 2013, MNRAS, 435, 1680

Kasen, D., Röpke, F. K., \& Woosley, S. E. 2009, Nature, 460, 869

Kasen, D., \& Woosley, S. E. 2007, ApJ, 656, 661

Kelly, P. L., Filippenko, A. V., Burke, D. L., Hicken, M., Ganeshalingam, M., \& Zheng, W. 2015, Science, 347, 1459

Kelly, P. L., Hicken, M., Burke, D. L., Mandel, K. S., \& Kirshner, R. P. 2010, ApJ, 715, 743

Kent, B. R., et al. 2008, AJ, 136, 713

Kerzendorf, W. E., Childress, M., Scharwächter, J., Do, T., \& Schmidt, B. P. 2014a, ApJ, 782, 27

Kerzendorf, W. E., Schmidt, B. P., Laird, J. B., Podsiadlowski, P., \& Bessell, M. S. 2012, ApJ, 759, 7

Kerzendorf, W. E., Taubenberger, S., Seitenzahl, I. R., \& Ruiter, A. J. 2014b, ApJL, 796, L26

Kerzendorf, W. E., et al. 2013, ApJ, 774, 99

Kessler, R., et al. 2009, ApJS, 185, 32

Konishi, K., et al. 2011, arXiv:1101.4269

Koribalski, B. S., et al. 2004, AJ, 128, 16

Kotak, R., et al. 2005, A\&A, 436, 1021

Krumm, N., \& Salpeter, E. E. 1980, AJ, 85, 1312

Kuchner, M. J., Kirshner, R. P., Pinto, P. A., \& Leibundgut, B. 1994, ApJL, 426, L89

Lampeitl, H., et al. 2010, ApJ, 722, 566

Landolt, A. U. 1992, AJ, 104, 372

Lauberts, A., \& Valentijn, E. A. 1989, The surface photometry catalogue of the ESO-Uppsala galaxies Leibundgut, B., et al. 1993, AJ, 105, 301

Leloudas, G., et al. 2009, A\&A, 505, 265

Leonard, D. C. 2007, ApJ, 670, 1275

Li, W., et al. 2011, Nature, 480, 348

Lira, P., et al. 1998, AJ, 115, 234

Livne, E. 1990, ApJL, 354, L53 
Lorén-Aguilar, P., Isern, J., \& García-Berro, E. 2010, MNRAS, 406, 2749

Lundqvist, P., et al. 2015, arXiv:1502.00589

Maeda, K., Taubenberger, S., Sollerman, J., Mazzali, P. A., Leloudas, G., Nomoto, K., \& Motohara, K. 2010a, ApJ, 708, 1703

Maeda, K., et al. 2010b, Nature, 466, 82

-. 2011, MNRAS, 413, 3075

Maguire, K., et al. 2012, MNRAS, 426, 2359

-. 2013, MNRAS, 436, 222

-. 2014, MNRAS, 444, 3258

Mannucci, F., Della Valle, M., \& Panagia, N. 2006, MNRAS, 370, 773

Mannucci, F., Della Valle, M., Panagia, N., Cappellaro, E., Cresci, G., Maiolino, R., Petrosian, A., \& Turatto, M. 2005, A\&A, 433, 807

Marion, G. H., et al. 2013, ApJ, 777, 40

Matheson, T., et al. 2008, AJ, 135, 1598

Maund, J. R., et al. 2010, ApJL, 725, L167

Mazzali, P. A., Cappellaro, E., Danziger, I. J., Turatto, M., \& Benetti, S. 1998, ApJL, 499, L49

Mazzali, P. A., Chugai, N., Turatto, M., Lucy, L. B., Danziger, I. J., Cappellaro, E., della Valle, M., \& Benetti, S. 1997, MNRAS, 284, 151

Mazzali, P. A., Maurer, I., Stritzinger, M., Taubenberger, S., Benetti, S., \& Hachinger, S. 2011, MNRAS, 416, 881

Mazzali, P. A., Röpke, F. K., Benetti, S., \& Hillebrandt, W. 2007, Science, 315, 825

Mazzali, P. A., Sauer, D. N., Pastorello, A., Benetti, S., \& Hillebrandt, W. 2008, MNRAS, 386, 1897

Mazzali, P. A., et al. 2005, ApJL, 623, L37

-. 2014, MNRAS, 439, 1959

-. 2015, arXiv:1504.04857

McClelland, C. M., Garnavich, P. M., Milne, P. A., Shappee, B. J., \& Pogge, R. W. 2013, ApJ, 767, 119

McCully, C., et al. 2014a, Nature, 512, 54

-. 2014b, ApJ, 786, 134

Modigliani, A., et al. 2010, in Society of Photo-Optical Instrumentation Engineers (SPIE) Conference Series, Vol. 7737, Society of Photo-Optical Instrumentation Engineers (SPIE) Conference Series, 28

Moll, R., Raskin, C., Kasen, D., \& Woosley, S. E. 2014, ApJ, 785, 105

Munari, U., Henden, A., Belligoli, R., Castellani, F., Cherini, G.,

Righetti, G. L., \& Vagnozzi, A. 2013, NewA, 20, 30

Nomoto, K. 1982, ApJ, 253, 798

Nugent, P. E., et al. 2011, Nature, 480, 344

Ogando, R. L. C., Maia, M. A. G., Pellegrini, P. S., \& da Costa, L. N. 2008, AJ, 135, 2424

Olling, R. P., et al. 2015, Nature, 521, 332

Pakmor, R., Kromer, M., Röpke, F. K., Sim, S. A., Ruiter, A. J., \& Hillebrandt, W. 2010, Nature, 463, 61

Pakmor, R., Kromer, M., Taubenberger, S., \& Springel, V. 2013, ApJL, 770, L8

Pan, Y.-C., Sullivan, M., Maguire, K., Gal-Yam, A., Hook, I. M., Howell, D. A., Nugent, P. E., \& Mazzali, P. A. 2015a, MNRAS, 446, 354

Pan, Y.-C., et al. 2015b, arXiv:1504.02396

Pastorello, A., et al. 2007, MNRAS, 377, 1531

Patat, F., Benetti, S., Cappellaro, E., Danziger, I. J., della Valle, M., Mazzali, P. A., \& Turatto, M. 1996, MNRAS, 278, 111

Patat, F., et al. 2007, Science, 317, 924
Pereira, R., et al. 2013, A\&A, 554, A27

Perlmutter, S., et al. 1999, ApJ, 517, 565

Phillips, M. M. 1993, ApJL, 413, L105

Phillips, M. M., Wells, L. A., Suntzeff, N. B., Hamuy, M., Leibundgut, B., Kirshner, R. P., \& Foltz, C. B. 1992, AJ, 103, 1632

Pignata, G., et al. 2004, MNRAS, 355, 178

-. 2008, MNRAS, 388, 971

Piro, A. L., \& Nakar, E. 2013, ApJ, 769, 67

Piro, A. L., Thompson, T. A., \& Kochanek, C. S. 2014, MNRAS, 438, 3456

Pisano, D. J., Barnes, D. G., Staveley-Smith, L., Gibson, B. K., Kilborn, V. A., \& Freeman, K. C. 2011, ApJS, 197, 28

Raskin, C., Kasen, D., Moll, R., Schwab, J., \& Woosley, S. 2014, ApJ, 788, 75

Rest, A., et al. 2014, ApJ, 795, 44

Rhee, M.-H., \& van Albada, T. S. 1996, A\&AS, 115, 407

Richmond, M. W., \& Smith, H. A. 2012, Journal of the American Association of Variable Star Observers (JAAVSO), 40, 872

Riess, A. G., et al. 1998, AJ, 116, 1009

-. 1999, AJ, 117, 707

-. 2005, ApJ, 627, 579

-. 2011, ApJ, 730, 119

Rigault, M., et al. 2013, A\&A, 560, A66

Röpke, F. K., \& Niemeyer, J. C. 2007, A\&A, 464, 683

Röpke, F. K., et al. 2012, ApJL, 750, L19

Rothberg, B., \& Joseph, R. D. 2006, AJ, 131, 185

Ruiter, A. J., et al. 2013, MNRAS, 429, 1425

Ruiz-Lapuente, P., \& Spruit, H. C. 1998, ApJ, 500, 360

Salvo, M. E., Cappellaro, E., Mazzali, P. A., Benetti, S., Danziger, I. J., Patat, F., \& Turatto, M. 2001, MNRAS, 321, 254

Sasdelli, M., Mazzali, P. A., Pian, E., Nomoto, K., Hachinger, S., Cappellaro, E., \& Benetti, S. 2014, MNRAS, 445, 711

Savitzky, A., \& Golay, M. J. E. 1964, Analytical Chemistry, 36, 1627

Scalzo, R., et al. 2012, ApJ, 757, 12

-. 2014a, MNRAS, 440, 1498

Scalzo, R. A., Ruiter, A. J., \& Sim, S. A. 2014b, MNRAS, 445, 2535

Scannapieco, E., \& Bildsten, L. 2005, ApJL, 629, L85

Scarano, S., Madsen, F. R. H., Roy, N., \& Lépine, J. R. D. 2008, MNRAS, 386, 963

Schaefer, B. E., \& Pagnotta, A. 2012, Nature, 481, 164

Schmidt, B. P., Kirshner, R. P., Leibundgut, B., Wells, L. A., Porter, A. C., Ruiz-Lapuente, P., Challis, P., \& Filippenko, A. V. 1994, ApJL, 434, L19

Schneider, S. E., Thuan, T. X., Mangum, J. G., \& Miller, J. 1992, ApJS, 81, 5

Scolnic, D. M., Riess, A. G., Foley, R. J., Rest, A., Rodney, S. A., Brout, D. J., \& Jones, D. O. 2014, ApJ, 780, 37

Seitenzahl, I. R., Cescutti, G., Röpke, F. K., Ruiter, A. J., \& Pakmor, R. 2013a, A\&A, 559, L5

Seitenzahl, I. R., Meakin, C. A., Townsley, D. M., Lamb, D. Q., \& Truran, J. W. 2009a, ApJ, 696, 515

Seitenzahl, I. R., Taubenberger, S., \& Sim, S. A. 2009b, MNRAS, 400, 531

Seitenzahl, I. R., et al. 2013b, MNRAS, 429, 1156

Shappee, B. J., \& Stanek, K. Z. 2011, ApJ, 733, 124

Shappee, B. J., Stanek, K. Z., Pogge, R. W., \& Garnavich, P. M. 2013, ApJL, 762, L5

Silbermann, N. A., et al. 1999, ApJ, 515, 1

Silverman, J. M., Ganeshalingam, M., \& Filippenko, A. V. 2013a, MNRAS, 430, 1030 
Silverman, J. M., Vinko, J., Marion, G. H., Wheeler, J. C., Barna, B., Szalai, T., Mulligan, B., \& Filippenko, A. V. 2015, arXiv: 1502.07278

Silverman, J. M., et al. 2012a, MNRAS, 425, 1789

-. 2012b, ApJL, 756, L7

-. 2013b, ApJ, 772, 125

-. 2013c, ApJS, 207, 3

Sim, S. A., Röpke, F. K., Hillebrandt, W., Kromer, M., Pakmor, R., Fink, M., Ruiter, A. J., \& Seitenzahl, I. R. 2010, ApJL, 714, L52

Sim, S. A., et al. 2013, MNRAS, 436, 333

Simon, J. D., et al. 2009, ApJ, 702, 1157

Smartt, S. J., et al. 2015, A\&A, 579, A40

Smith, J. A., et al. 2002, AJ, 123, 2121

Sollerman, J., et al. 2004, A\&A, 428, 555

Spyromilio, J., Gilmozzi, R., Sollerman, J., Leibundgut, B., Fransson, C., \& Cuby, J.-G. 2004, A\&A, 426, 547

Stanishev, V., et al. 2007, A\&A, 469, 645

Stehle, M., Mazzali, P. A., Benetti, S., \& Hillebrandt, W. 2005, MNRAS, 360, 1231

Strauss, M. A., Huchra, J. P., Davis, M., Yahil, A., Fisher, K. B., \& Tonry, J. 1992, ApJS, 83, 29

Stritzinger, M., Leibundgut, B., Walch, S., \& Contardo, G. 2006a, A\&A, 450, 241

Stritzinger, M., Mazzali, P. A., Sollerman, J., \& Benetti, S. 2006b, A\&A, 460, 793

Stritzinger, M., \& Sollerman, J. 2007, A\&A, 470, L1

Stritzinger, M. D., et al. 2011, AJ, 142, 156

Sullivan, M., et al. 2006, ApJ, 648, 868

-. 2010, MNRAS, 406, 782

—. 2011, ApJ, 737, 102

Sunderland, A. G., Noble, C. J., Burke, V. M., \& Burke, P. G. 2002, Computer Physics Communications, 145, 311

Suntzeff, N. B., et al. 1999, AJ, 117, 1175

Tanaka, M., Mazzali, P. A., Stanishev, V., Maurer, I., Kerzendorf,

W. E., \& Nomoto, K. 2011, MNRAS, 410, 1725

Taubenberger, S., et al. 2013, MNRAS, 432, 3117

-. 2015, MNRAS, 448, L48

Theureau, G., Bottinelli, L., Coudreau-Durand, N., Gouguenheim, L., Hallet, N., Loulergue, M., Paturel, G., \& Teerikorpi, P. 1998, A\&AS, 130, 333

Trager, S. C., Faber, S. M., Worthey, G., \& González, J. J. 2000, AJ, 119, 1645

Tutukov, A. V., \& Iungelson, L. R. 1976, Astrofizika, 12, 521

Tutukov, A. V., \& Yungelson, L. R. 1979, AcA, 29, 665

Verheijen, M. A. W., \& Sancisi, R. 2001, A\&A, 370, 765

Vernet, J., et al. 2011, A\&A, 536, A105

Wang, X., Li, W., Filippenko, A. V., Foley, R. J., Smith, N., \&

Wang, L. 2008a, ApJ, 677, 1060

Wang, X., et al. 2008b, ApJ, 675, 626

-. 2009, ApJ, 697, 380

Webbink, R. F. 1984, ApJ, 277, 355

Whelan, J., \& Iben, Jr., I. 1973, ApJ, 186, 1007

Wong, O. I., et al. 2006, MNRAS, 371, 1855

Woosley, S. E., \& Kasen, D. 2011, ApJ, 734, 38

Woosley, S. E., \& Weaver, T. A. 1994, ApJ, 423, 371

Yamanaka, M., et al. 2014, ApJL, 782, L35

Yaron, O., \& Gal-Yam, A. 2012, PASP, 124, 668

Zhang, J.-J., Wang, X.-F., Bai, J.-M., Zhang, T.-M., Wang, B., Liu, Z.-W., Zhao, X.-L., \& Chen, J.-C. 2014, AJ, 148, 1

Zhang, T., et al. 2010, PASP, 122, 1
Table A1. Atomic data for Co III

\begin{tabular}{llccc}
\hline \hline Lower level & Upper level & $\mathrm{A}^{a}$ & $\lambda$ & $\mathrm{E}(\mathrm{eV})$ \\
\hline $3 \mathrm{~d}^{7} \mathrm{a}^{4} \mathrm{~F}_{9 / 2}$ & $3 \mathrm{~d}^{7} \mathrm{a}^{2} \mathrm{G}_{7 / 2}$ & 0.014 & 5627.104 & 2.203 \\
$3 \mathrm{~d}^{7} \mathrm{a}^{4} \mathrm{~F}_{9 / 2}$ & $3 \mathrm{~d}^{7} \mathrm{a}^{2} \mathrm{G}_{9 / 2}$ & 0.400 & 5888.482 & 2.105 \\
$3 \mathrm{~d}^{7} \mathrm{a}^{4} \mathrm{~F}_{7 / 2}$ & $3 \mathrm{~d}^{7} \mathrm{a}^{2} \mathrm{G}_{7 / 2}$ & 0.150 & 5906.783 & 2.203 \\
$3 \mathrm{~d}^{7} \mathrm{a}^{4} \mathrm{~F}_{7 / 2}$ & $3 \mathrm{~d}^{7} \mathrm{a}^{2} \mathrm{G}_{9 / 2}$ & 0.120 & 6195.455 & 2.105 \\
$3 \mathrm{~d}^{7} \mathrm{a}^{4} \mathrm{~F}_{5 / 2}$ & $3 \mathrm{~d}^{7} \mathrm{a}^{2} \mathrm{G}_{7 / 2}$ & 0.110 & 6127.670 & 2.203 \\
\hline
\end{tabular}

${ }^{a}$ The $A$ values are from Hansen et al. (1984)

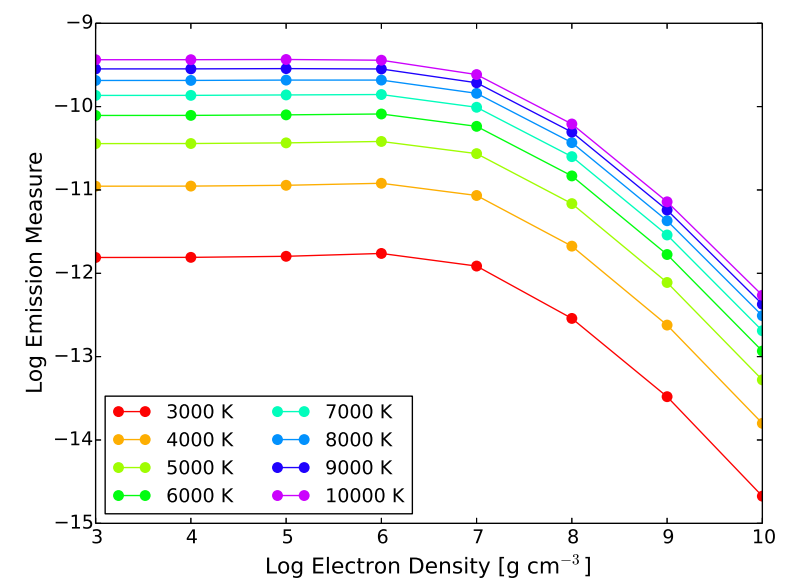

Figure A1. Illustration of the variation of the flux in the [Co III] $\lambda 5893$ blend with electron density. The different curves are for temperatures of $3000 \mathrm{~K}$ (red curve) to $10000 \mathrm{~K}$ (top curve) in steps of $1000 \mathrm{~K}$. For the calculation a $\mathrm{SN}$ age of 100 days and a $\mathrm{N}(\mathrm{CoIII})$ to $\mathrm{N}(\mathrm{Ne})$ ratio of 0.01 was assumed. The emission measure has been divided by the product of the electron and Co densities.

\section{APPENDIX A: ATOMIC DATA FOR Co III}

Rest wavelength: Table A1 lists the transitions in the multiplet of Co III contributing to the $5893 \AA$ feature. Note that the second and third transitions contribute to the main $5893 \AA$, while other transitions in the multiplet produce other features of interest such as the $\sim 6150 \AA$ feature of Co III (see Figure 2).

Line emissivity: The [Co III] $\lambda 5893$ blend accounts for about $70 \%$ of the multiplet cooling. Above an electron density of $10^{7}$ $\mathrm{cm}^{-3}$ and a temperature of $4000 \mathrm{~K}$, the above multiplet accounts for between 50 and $70 \%$ of all cooling by Co III. As the temperature and/or electron density is lowered below these values, cooling via the $3 d^{7} a^{4} \mathrm{~F}-3 d^{7} a^{4} \mathrm{P}$ multiplet (with an excitation energy of $\sim 1.9 \mathrm{eV}$ ) becomes increasingly important. Plots of the flux in the [Co III] $\lambda 5893$ blend as a function of electron density and temperature are shown in Fig. A1 The critical density for the $3 d^{7} a^{2} G$ levels is $\sim 3 \times 10^{7}$ and for the $3 \mathrm{~d}^{7} \mathrm{a}^{4} \mathrm{P}$ levels it is $\sim 1 \times 10^{6}$. We note here that collision rates for Co III are unavailable and so we adopted collision strengths from Sunderland et al. (2002) which were computed for Ni IV, and which has a similar electronic structure to Co III. 


\section{APPENDIX B: ANCILLARY DATA ON SN SAMPLE}

This Appendix presents the properties of SNe Ia and their host galaxies derived in the literature or measured from the data for our analysis.

Table B1 presents the host galaxy distance and redshift information for the SNe Ia in our sample, along with references. As noted in Section 3 distances for SNe Ia in our sample are preferentially derived from Cepheid distances from Riess et al. (2011) where available, and from host redshift with the Riess et al. (2011) Hubble constant value $\left(H_{0}=73.8 \mathrm{~km} \mathrm{~s}^{-1} \mathrm{Mpc}^{-1}\right)$. Generally we avoid other distance indicators such as Tully-Fisher, surface brightness fluctuations, and planetary nebula luminosity functions. The one exception to this is SN 2012cg, for which we use the Cortés et al. (2008) Tully-Fisher distance modulus, as was done in Silverman et al. (2012b).

For some SNe Ia (SN 2003du, SN 2011by, SN 2013dy), previous analyses in the literature have found that the redshift-based distance modulus yields anomalous values for the SN peak luminosity. Thus for these we employ SN-based distance moduli adopted by previous authors: the Stritzinger et al. (2006b) value for SN 2003du, the Graham et al. (2015) value for SN 2011by, and the Pan et al. (2015a) value for SN 2013dy (derived with SNooPy).

Table B2 presents the pertinent light curve fit parameters from the main photospheric phase light curves. Light curves were fit with SiFTO (Conley et al. 2008). For some SNe Ia (SN 2011iv, SN 2011by, SN 2012ht), photometry was not explicitly published, so we utilize the published light curve widths and colours for these SNe. Where appropriate, we convert $\Delta m_{15}(B)$ or SALT2 $x 1$ to SiFTO stretch $s$ using the relations published in Guy et al. (2007, 2010).

Light curves for SN 2013cs and SN 2013gy were obtained with the Las Cumbres Observatories Global Telescope (LCOGT Brown et al. 2013) and have been reduced using a custom pipeline developed by S. Valenti. The pipeline employs standard procedures (PyRAF, DAOPHOT) in a Python framework. Host galaxy flux was removed using a low order polynomial background. Point spread function magnitudes were transformed to the standard Sloan Digital Sky Survey (Smith et al. 2002) filter system (for gri) or Landolt (1992) system (for BV) via standard star observations taken during clear nights. These light curves will be released in a future LCOGT supernova program publication.

Table B3 presents the late-time photometry for our SNe Ia. The $B$-band magnitudes for each phase of spectroscopic observation was determined from a linear fit to the very late $(t \geq 60$ days past peak) $B$-band light curve. Spectra from T15b were already flux calibrated to contemporaneous photometry, so here we report the $B$-band flux synthesized from the spectrum. Similarly, M15 spectra were flux calibrated with spectrophotometric standard stars, and thus are flux calibrated modulo any changes in grey extinction or slit loss between the standard star and SN observation.

For some $\mathrm{SNe} \mathrm{Ia}$, sufficient late time photometry was not available to reliably extrapolate the $B$-band luminosity at the time of spectroscopic observations - generally this occurred if the SN did not have photometry beyond 100 days past peak or if a public light curve was not available. For these $\mathrm{SNe}$, late photometry from an alternate $\mathrm{SN}$ was employed, with the requirement that the light curve stretch be exceptionally close. These SNe with their surrogate light curves are shown in Figure B1 and the surrogate SN is denoted in the reference column of Table B3.

Table B4 presents the measured [Co III] $\lambda 5893$ line fluxes, synthesized $B$-band fluxes, and wavelength integration bounds for the [Co III] $\lambda 5893$ flux measurements. Note that the fluxes in this table are in the natural units of the spectroscopic data, as measured from its publicly available format. 
Table B1. Nebular SN Ia Host Information

\begin{tabular}{|c|c|c|c|c|c|c|}
\hline SN & $\begin{array}{l}\text { Host } \\
\text { Name }\end{array}$ & Host $z$ & $\begin{array}{l}\text { Host } \mu \\
\text { (mag) }^{a}\end{array}$ & $\begin{array}{l}\mu \\
\text { Method }\end{array}$ & $z$ Ref. & $\mu$ Ref. \\
\hline SN1990N & NGC 4639 & 0.003395 & $31.67 \pm 0.07$ & Cepheids & Wong et al. (2006) & Riess et al. (2011) \\
\hline SN1991T & NGC 4527 & 0.005791 & $30.72 \pm 0.13$ & Cepheids & Strauss et al. (1992) & NED \\
\hline SN1994D & NGC 4526 & 0.002058 & $29.73 \pm 1.06$ & Redshift & Cappellari et al. (2011) & $\ldots$ \\
\hline SN1994ae & NGC 3370 & 0.004266 & $32.13 \pm 0.07$ & Cepheids & Krumm \& Salpeter (1980) & Riess et al. (2011) \\
\hline SN1995D & NGC 2962 & 0.006561 & $32.25 \pm 0.33$ & Redshift & Cappellari et al. (2011) & $\cdots$ \\
\hline SN1998aq & NGC 3982 & 0.003699 & $31.70 \pm 0.08$ & Cepheids & de Vaucouleurs et al. (1991) & Riess et al. (2011) \\
\hline SN1998bu & NGC 3368 & 0.002992 & $30.20 \pm 0.20$ & Cepheids & de Vaucouleurs et al. (1991) & NED \\
\hline SN1999aa & NGC 2595 & 0.014443 & $33.98 \pm 0.15$ & Redshift & de Vaucouleurs et al. (1991) & $\ldots$ \\
\hline SN2002cs & NGC 6702 & 0.015771 & $34.17 \pm 0.14$ & Redshift & Trager et al. (2000) & $\cdots$ \\
\hline SN2002dj & NGC 5018 & 0.009393 & $33.04 \pm 0.23$ & Redshift & Rothberg \& Joseph (2006) & $\cdots$ \\
\hline SN2002er & UGC 10743 & 0.008569 & $32.84 \pm 0.25$ & Redshift & de Vaucouleurs et al. (1991) & $\ldots$ \\
\hline SN2002fk & NGC 1309 & 0.007125 & $32.59 \pm 0.09$ & Cepheids & Koribalski et al. (2004) & Riess et al. (2011) \\
\hline SN2003du & UGC 9391 & 0.006384 & $32.75 \pm 0.20$ & SN & Schneider et al. (1992) & Stritzinger et al. (2006b) \\
\hline SN2003hv & NGC 1201 & 0.005624 & $31.92 \pm 0.39$ & Redshift & Ogando et al. (2008) & $\cdots$ \\
\hline SN2004bv & NGC 6907 & 0.010614 & $33.31 \pm 0.20$ & Redshift & Scarano et al. (2008) & $\cdots$ \\
\hline SN2004eo & NGC 6928 & 0.015701 & $34.16 \pm 0.14$ & Redshift & Theureau et al. (1998) & $\cdots$ \\
\hline SN2005am & NGC 2811 & 0.007899 & $32.66 \pm 0.28$ & Redshift & Theureau et al. (1998) & $\cdots$ \\
\hline SN2005cf & MCG-01-39-3 & 0.006430 & $32.21 \pm 0.34$ & Redshift & Childress et al. (2013a) & $\cdots$ \\
\hline SN2007af & NGC 5584 & 0.005464 & $31.72 \pm 0.07$ & Cepheids & Koribalski et al. (2004) & Riess et al. (2011) \\
\hline SN2007gi & NGC 4036 & 0.004620 & $31.49 \pm 0.47$ & Redshift & Cappellari et al. (2011) & $\cdots$ \\
\hline SN2007le & NGC 7721 & 0.006721 & $32.31 \pm 0.32$ & Redshift & Koribalski et al. (2004) & $\cdots$ \\
\hline SN2007sr & NGC 4038 & 0.005477 & $31.66 \pm 0.08$ & Cepheids & Lauberts \& Valentiin (1989) & Riess et al. (2011) \\
\hline SN2008Q & NGC 524 & 0.008016 & $32.69 \pm 0.27$ & Redshift & Cappellari et al. (2011) & $\cdots$ \\
\hline SN2009ig & NGC 1015 & 0.008770 & $32.89 \pm 0.25$ & Redshift & Wong et al. (2006) & $\ldots$ \\
\hline SN2009le & MCG-04-06-9 & 0.017786 & $34.44 \pm 0.12$ & Redshift & Theureau et al. (1998) & $\cdots$ \\
\hline SN2011by & NGC 3972 & 0.002843 & $32.01 \pm 0.07$ & $\mathrm{SN}$ & Verheiien \& Sancisi (2001) & Graham et al. (2015) \\
\hline SN2011ek & NGC 918 & 0.005027 & $31.67 \pm 0.43$ & Redshift & Rhee \& van Albada (1996) & $\cdots$ \\
\hline SN2011fe & NGC 5457 & 0.000804 & $29.05 \pm 0.06$ & Cepheids & de Vaucouleurs et al. (1991) & Shappee \& Stanek (2011) \\
\hline SN2011iv & NGC 1404 & 0.006494 & $32.23 \pm 0.33$ & Redshift & Graham et al. (1998) & $\cdots$ \\
\hline SN2012cg & NGC 4424 & 0.001458 & $30.90 \pm 0.30$ & $\mathrm{TF}$ & Kent et al. (2008) & Cortés et al. (2008) \\
\hline SN2012fr & NGC 1365 & 0.005457 & $31.31 \pm 0.20$ & Cepheids & Bureau et al. (1996) & Silbermann et al. (1999) \\
\hline SN2012hr & ESO 121-G026 & 0.007562 & $32.56 \pm 0.29$ & Redshift & Koribalski et al. (2004) & $\cdots$ \\
\hline SN2012ht & NGC 3447 & 0.003559 & $30.92 \pm 0.61$ & Redshift & Guthrie \& Napier (1996) & $\cdots$ \\
\hline SN2013aa & NGC 5643 & 0.003999 & $31.18 \pm 0.54$ & Redshift & Koribalski et al. (2004) & $\cdots$ \\
\hline SN2013cs & ESO 576-G017 & 0.009243 & $33.00 \pm 0.24$ & Redshift & Pisano et al. (2011) & $\cdots$ \\
\hline SN2013dy & NGC 7250 & 0.003889 & $31.49 \pm 0.10$ & SN & Schneider et al. (1992) & Pan et al. (2015a) \\
\hline SN2013gy & NGC 1418 & 0.014023 & $33.92 \pm 0.15$ & Redshift & Catinella et al. (2005) & $\cdots$ \\
\hline SN2014J & NGC 3034 & 0.000677 & $27.60 \pm 0.10$ & Cepheids+SN & de Vaucouleurs et al. (1991) & Foley et al. (2014) \\
\hline
\end{tabular}

${ }^{a}$ For hosts with redshift-based $\mu$, uncertainty includes a peculiar velocity term of $300 \mathrm{~km} \mathrm{~s}^{-1}$. 
Table B2. Light Curve Fit Results and Sources

\begin{tabular}{|c|c|c|c|c|c|}
\hline SN & $\begin{array}{l}\text { MJD } \\
\text { of } B_{\max }\end{array}$ & $\begin{array}{l}\text { Rest Frame } \\
B_{\max }(\mathrm{mag})\end{array}$ & Stretch & Color $c$ & $\begin{array}{l}\text { LC } \\
\text { Ref. }\end{array}$ \\
\hline SN1990N & 48081.69 & $12.706 \pm 0.015$ & $1.081 \pm 0.012$ & $0.049 \pm 0.013$ & Lira et al. (1998) \\
\hline SN1991T & 48373.95 & $11.468 \pm 0.028$ & $1.047 \pm 0.019$ & $0.101 \pm 0.026$ & Lira et al. (1998) \\
\hline SN1994D & 49431.52 & $11.775 \pm 0.009$ & $0.823 \pm 0.004$ & $-0.099 \pm 0.005$ & Patat et al. (1996) \\
\hline SN1994ae & 49684.56 & $12.968 \pm 0.019$ & $1.057 \pm 0.014$ & $-0.060 \pm 0.016$ & CfA \\
\hline SN1995D & 49767.50 & $13.273 \pm 0.025$ & $1.097 \pm 0.014$ & $-0.006 \pm 0.015$ & CfA \\
\hline SN1998aq & 50930.24 & $12.316 \pm 0.009$ & $0.965 \pm 0.010$ & $-0.146 \pm 0.009$ & Riess et al. (2005) \\
\hline SN1998bu & 50952.06 & $12.118 \pm 0.013$ & $0.962 \pm 0.018$ & $0.270 \pm 0.010$ & Jha et al. (1999); Suntzeff et al. (1999) \\
\hline SN1999aa & 51231.84 & $14.755 \pm 0.016$ & $1.134 \pm 0.009$ & $-0.047 \pm 0.009$ & CfA \\
\hline SN2002cs & 52409.24 & $15.138 \pm 0.039$ & $1.007 \pm 0.013$ & $0.017 \pm 0.016$ & LOSS \\
\hline SN2002dj & 52450.32 & $13.974 \pm 0.035$ & $0.962 \pm 0.013$ & $0.098 \pm 0.016$ & LOSS \\
\hline SN2002er & 52524.03 & $14.267 \pm 0.057$ & $0.901 \pm 0.009$ & $0.123 \pm 0.018$ & LOSS \\
\hline SN2002fk & 52547.28 & $13.152 \pm 0.017$ & $0.995 \pm 0.012$ & $-0.142 \pm 0.012$ & LOSS \\
\hline SN2003du & 52765.48 & $13.476 \pm 0.007$ & $1.016 \pm 0.008$ & $-0.110 \pm 0.008$ & LOSS \\
\hline SN2003hv & 52891.12 & $12.444 \pm 0.020$ & $0.741 \pm 0.021$ & $-0.115 \pm 0.014$ & Leloudas et al. (2009) \\
\hline SN2004bv & 53159.83 & $13.938 \pm 0.024$ & $1.146 \pm 0.014$ & $0.122 \pm 0.013$ & LOSS \\
\hline SN2004eo & 53277.66 & $15.099 \pm 0.038$ & $0.863 \pm 0.009$ & $0.002 \pm 0.014$ & CSP \\
\hline SN2005am & 53436.97 & $13.698 \pm 0.023$ & $0.710 \pm 0.019$ & $0.057 \pm 0.012$ & CSP \\
\hline SN2005cf & 53533.28 & $13.625 \pm 0.035$ & $0.968 \pm 0.009$ & $0.021 \pm 0.013$ & LOSS \\
\hline SN2007af & 54173.88 & $13.180 \pm 0.015$ & $0.955 \pm 0.011$ & $0.058 \pm 0.010$ & CSP \\
\hline SN2007gi & 54327.66 & $13.158 \pm 0.016$ & $0.871 \pm 0.013$ & $0.097 \pm 0.017$ & Zhang et al. (2010) \\
\hline SN2007le & 54398.62 & $13.876 \pm 0.016$ & $1.028 \pm 0.015$ & $0.342 \pm 0.014$ & CSP \\
\hline $\mathrm{SN} 2007 \mathrm{sr}$ & 54448.70 & $12.809 \pm 0.042$ & $0.970 \pm 0.031$ & $0.141 \pm 0.016$ & LOSS \\
\hline SN2008Q & 54505.47 & $13.510 \pm 0.033$ & $0.803 \pm 0.025$ & $0.001 \pm 0.018$ & LOSS \\
\hline SN2009ig & 55079.18 & $13.437 \pm 0.019$ & $1.136 \pm 0.028$ & $0.055 \pm 0.019$ & CfA \\
\hline SN2009le & 55165.16 & $15.351 \pm 0.030$ & $1.022 \pm 0.022$ & $0.079 \pm 0.033$ & CfA \\
\hline SN2011by & 55690.60 & $12.890 \pm 0.030$ & $0.959 \pm 0.019$ & $0.000 \pm 0.050$ & Graham et al. (2015) \\
\hline SN2011ek & 55788.90 & $13.840 \pm 0.150$ & $0.900 \pm 0.020$ & $0.180 \pm 0.040$ & Maguire et al. (2013) \\
\hline SN2011fe & 55814.51 & $9.940 \pm 0.010$ & $0.969 \pm 0.010$ & $-0.066 \pm 0.021$ & Pereira et al. (2013) \\
\hline SN2011iv & 55906.00 & $12.530 \pm 0.040$ & $0.684 \pm 0.020$ & $0.000 \pm 0.050$ & Foley et al. (2012) \\
\hline SN2012cg & 56082.03 & $12.128 \pm 0.011$ & $1.063 \pm 0.011$ & $0.184 \pm 0.010$ & Munari et al. (2013) \\
\hline SN2012fr & 56243.68 & $12.017 \pm 0.013$ & $1.108 \pm 0.008$ & $0.059 \pm 0.011$ & Zhang et al. (2014) \\
\hline SN2012hr & 56289.20 & $13.780 \pm 0.020$ & $1.018 \pm 0.021$ & $0.030 \pm 0.010$ & Maguire et al. (2013) \\
\hline SN2012ht & 56295.60 & $13.220 \pm 0.040$ & $0.818 \pm 0.025$ & $0.000 \pm 0.050$ & Yamanaka et al. (2014) \\
\hline SN2013aa & 56344.00 & $11.330 \pm 0.050$ & $1.146 \pm 0.019$ & $-0.050 \pm 0.010$ & Maguire et al. (2013) \\
\hline SN2013cs & 56436.95 & $13.659 \pm 0.032$ & $1.006 \pm 0.010$ & $0.054 \pm 0.015$ & LCOGT \\
\hline SN2013dy & 56500.65 & $12.824 \pm 0.048$ & $1.101 \pm 0.007$ & $0.195 \pm 0.015$ & Pan et al. (2015b) \\
\hline SN2013gy & 56648.61 & $14.742 \pm 0.020$ & $0.948 \pm 0.008$ & $0.070 \pm 0.011$ & LCOGT \\
\hline SN2014J & 56689.74 & $11.850 \pm 0.012$ & $1.086 \pm 0.010$ & $1.251 \pm 0.012$ & Foley et al. (2014) \\
\hline
\end{tabular}

CfA: Riess et al. (1999); Jha et al. (2006); Hicken et al. (2009, 2012); LOSS: Ganeshalingam et al. 2010); CSP: Contreras et al. 2010); Stritzinger et al. (2011) 

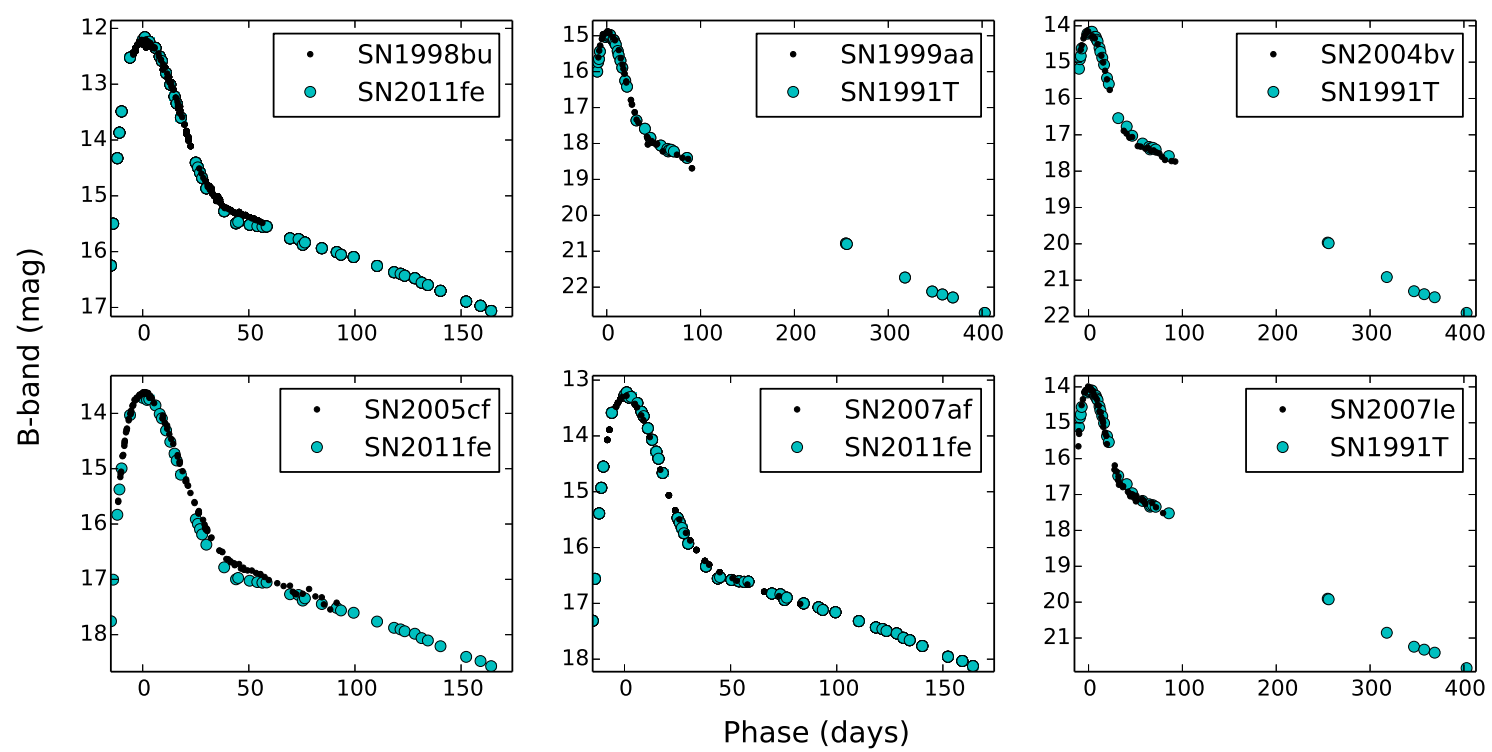

Figure B1. Surrogate light curves for SNe Ia with poor late time light curve coverage. Original SN data are shown as small black dots while surrogate SN data are shown as larger blue circles. 
Table B3. Late Phase Photometry

\begin{tabular}{|c|c|c|c|c|}
\hline $\mathrm{SN}$ & $\begin{array}{c}\text { Phase } t \\
\text { (days) }\end{array}$ & Obs. Date & $\begin{array}{c}m_{B}(t) \\
(\mathrm{mag})\end{array}$ & Phot. Ref. \\
\hline SN1990N & 160 & 19901217 & $17.394 \pm 0.014$ & Lira et al. (1998) \\
\hline SN1990N & 186 & 19910112 & $17.760 \pm 0.011$ & \\
\hline SN1990N & 227 & 19910222 & $18.337 \pm 0.007$ & \\
\hline SN1990N & 255 & 19910322 & $18.731 \pm 0.008$ & \\
\hline SN1990N & 280 & 19910416 & $19.083 \pm 0.011$ & \\
\hline SN1990N & 333 & 19910608 & $19.829 \pm 0.018$ & \\
\hline SN1991T & 113 & 19910819 & $17.026 \pm 0.032$ & Schmidt et al. (1994) \\
\hline SN1991T & 186 & 19911031 & $17.614 \pm 0.024$ & \\
\hline SN1991T & 258 & 19920111 & $18.194 \pm 0.018$ & \\
\hline SN1991T & 320 & 19920313 & $18.694 \pm 0.018$ & \\
\hline SN1991T & 349 & 19920411 & $18.928 \pm 0.019$ & \\
\hline SN1993Z & 173 & 19940317 & $19.064 \pm 0.063$ & Ho et al. (2001) \\
\hline SN1993Z & 205 & 19940418 & $19.588 \pm 0.094$ & \\
\hline SN1994D & 613 & 19951124 & $23.227 \pm 0.040$ & Altavilla et al. (2004) \\
\hline SN1994ae & 144 & 19950422 & $17.672 \pm 0.025$ & Altavilla et al. (2004) \\
\hline SN1994ae & 153 & 19950501 & $17.812 \pm 0.027$ & \\
\hline SN1995D & 277 & 19951124 & $19.887 \pm 0.041$ & Altavilla et al. (2004) \\
\hline SN1995D & 285 & 19951202 & $20.001 \pm 0.043$ & \\
\hline SN1996X & 298 & 19970210 & $20.108 \pm 0.022$ & Salvo et al. (2001) \\
\hline SN1998aq & 211 & 19981124 & $18.140 \pm 0.011$ & Riess et al. (2005) \\
\hline SN1998aq & 231 & 19981214 & $18.442 \pm 0.013$ & \\
\hline SN1998aq & 241 & 19981224 & $18.593 \pm 0.013$ & \\
\hline SN1998bu & 179 & 19981114 & $17.202 \pm 0.017$ & SN2011fe \\
\hline SN1998bu & 190 & 19981125 & $17.347 \pm 0.019$ & \\
\hline SN1998bu & 208 & 19981213 & $17.586 \pm 0.022$ & \\
\hline SN1998bu & 217 & 19981222 & $17.705 \pm 0.023$ & \\
\hline SN1998bu & 236 & 19990110 & $17.956 \pm 0.026$ & \\
\hline SN1998bu & 243 & 19990117 & $18.049 \pm 0.028$ & \\
\hline SN1998bu & 280 & 19990223 & $18.538 \pm 0.034$ & \\
\hline SN1998bu & 329 & 19990413 & $19.187 \pm 0.042$ & \\
\hline SN1998bu & 340 & 19990424 & $19.332 \pm 0.043$ & \\
\hline SN1999aa & 256 & 19991109 & $20.872 \pm 0.000$ & SN1991T \\
\hline SN1999aa & 282 & 19991205 & $21.236 \pm 0.000$ & \\
\hline SN2002cs & 174 & 20021106 & $20.264 \pm 0.080$ & LOSS \\
\hline SN2002dj & 222 & 20030201 & $20.143 \pm 0.058$ & Pignata et al. (2004) \\
\hline SN2002dj & 275 & 20030326 & $21.003 \pm 0.040$ & \\
\hline SN2002er & 216 & 20030410 & $20.429 \pm 0.022$ & Pignata et al. (2008) \\
\hline SN2002fk & 150 & 20030227 & $18.099 \pm 0.024$ & CfA, LOSS \\
\hline SN2003du & 109 & 20030823 & $17.612 \pm 0.004$ & Stanishev et al. (2007) \\
\hline SN2003du & 138 & 20030921 & $18.043 \pm 0.003$ & \\
\hline SN2003du & 139 & 20030922 & $18.058 \pm 0.003$ & \\
\hline SN2003du & 142 & 20030925 & $18.102 \pm 0.003$ & \\
\hline SN2003du & 209 & 20031201 & $19.096 \pm 0.004$ & \\
\hline SN2003du & 221 & 20031213 & $19.274 \pm 0.004$ & \\
\hline SN2003du & 272 & 20040202 & $20.031 \pm 0.005$ & \\
\hline SN2003du & 377 & 20040517 & $21.589 \pm 0.008$ & \\
\hline SN2003gs & 170 & 20040214 & $19.877 \pm 0.051$ & LOSS \\
\hline SN2003hv & 113 & 20031228 & $16.927 \pm 0.003$ & Leloudas et al. (2009) \\
\hline SN2003hv & 145 & 20040129 & $17.436 \pm 0.004$ & \\
\hline SN2003hv & 323 & 20040725 & $20.870 \pm 0.012$ & \\
\hline SN2004bv & 171 & 20041114 & $18.713 \pm 0.000$ & SN1991T \\
\hline SN2004eo & 228 & 20050516 & $21.454 \pm 0.008$ & Pastorello et al. (2007) \\
\hline SN2005cf & 319 & 20060427 & $20.558 \pm 0.040$ & SN2011fe \\
\hline SN2006X & 127 & 20060626 & $19.001 \pm 0.027$ & Wang et al. (2008b) \\
\hline SN2006X & 152 & 20060721 & $19.196 \pm 0.040$ & \\
\hline SN2006X & 277 & 20061123 & $20.175 \pm 0.107$ & \\
\hline SN2006X & 307 & 20061223 & $20.410 \pm 0.123$ & \\
\hline
\end{tabular}


Table B3 (cont'd)

\begin{tabular}{|c|c|c|c|c|}
\hline $\mathrm{SN}$ & $\begin{array}{c}\text { Phase } t \\
\text { (days) }\end{array}$ & Obs. Date & $\begin{array}{c}m_{B}(t) \\
(\mathrm{mag})\end{array}$ & Phot. Ref. \\
\hline SN2006X & 360 & 20070214 & $20.824 \pm 0.151$ & \\
\hline SN2007af & 103 & 20070620 & $17.181 \pm 0.005$ & SN2011fe \\
\hline SN2007af & 108 & 20070625 & $17.247 \pm 0.006$ & \\
\hline SN2007af & 120 & 20070707 & $17.406 \pm 0.007$ & \\
\hline SN2007af & 123 & 20070710 & $17.446 \pm 0.008$ & \\
\hline SN2007af & 128 & 20070715 & $17.512 \pm 0.008$ & \\
\hline SN2007af & 131 & 20070718 & $17.552 \pm 0.009$ & \\
\hline SN2007af & 151 & 20070807 & $17.816 \pm 0.012$ & \\
\hline SN2007af & 165 & 20070821 & $18.002 \pm 0.014$ & \\
\hline SN2007af & 308 & 20080111 & $19.894 \pm 0.037$ & \\
\hline SN2007gi & 161 & 20080115 & $17.920 \pm 0.014$ & Zhang et al. (2010) \\
\hline SN2007le & 317 & 20080827 & $20.653 \pm 0.000$ & SN1991T \\
\hline SN2007sr & 177 & 20080623 & $17.994 \pm 0.021$ & CfA, LOSS \\
\hline SN2009ig & 405 & 20101017 & $21.729 \pm 0.050$ & $\mathrm{CfA}$ \\
\hline SN2009le & 324 & 20101016 & $22.651 \pm 0.050$ & CfA \\
\hline SN2010ev & 178 & 20101231 & $20.199 \pm 0.050$ & $\mathrm{~T} 15 \mathrm{~b}$ \\
\hline SN2010ev & 272 & 20110404 & $21.183 \pm 0.050$ & \\
\hline SN2010gp & 279 & 20110501 & $23.150 \pm 0.050$ & $\mathrm{~T} 15 \mathrm{~b}$ \\
\hline SN2010hg & 199 & 20110403 & $19.765 \pm 0.050$ & $\mathrm{~T} 15 \mathrm{~b}$ \\
\hline SN2010kg & 289 & 20110927 & $22.592 \pm 0.050$ & $\mathrm{~T} 15 \mathrm{~b}$ \\
\hline SN2011K & 341 & 20111228 & $23.096 \pm 0.050$ & $\mathrm{~T} 15 \mathrm{~b}$ \\
\hline SN2011ae & 310 & 20120101 & $20.671 \pm 0.050$ & $\mathrm{~T} 15 \mathrm{~b}$ \\
\hline SN2011at & 349 & 20120227 & $21.656 \pm 0.050$ & $\mathrm{~T} 15 \mathrm{~b}$ \\
\hline SN2011by & 206 & 20111202 & $18.337 \pm 0.022$ & SN2011fe \\
\hline SN2011by & 310 & 20120315 & $19.713 \pm 0.039$ & \\
\hline SN2011ek & 423 & 20121011 & $24.328 \pm 0.050$ & $\mathrm{~T} 15 \mathrm{~b}$ \\
\hline SN2011fe & 074 & 20111123 & $13.630 \pm 0.004$ & Richmond \& Smith (2012) \\
\hline SN2011fe & 114 & 20120102 & $14.157 \pm 0.007$ & \\
\hline SN2011fe & 196 & 20120324 & $15.238 \pm 0.019$ & \\
\hline SN2011fe & 230 & 20120427 & $15.686 \pm 0.024$ & \\
\hline SN2011fe & 276 & 20120612 & $16.292 \pm 0.030$ & \\
\hline SN2011fe & 314 & 20120720 & $16.792 \pm 0.036$ & \\
\hline SN2011im & 314 & 20121016 & $23.522 \pm 0.050$ & $\mathrm{~T} 15 \mathrm{~b}$ \\
\hline SN2011iv & 318 & 20121024 & $20.717 \pm 0.050$ & $\mathrm{~T} 15 \mathrm{~b}$ \\
\hline SN2011jh & 414 & 20130221 & $24.131 \pm 0.050$ & $\mathrm{~T} 15 \mathrm{~b}$ \\
\hline SN2012cg & 330 & 20130507 & $19.824 \pm 0.085$ & SN1994ae \\
\hline SN2012cg & 342 & 20130513 & $19.917 \pm 0.087$ & \\
\hline SN2012cu & 340 & 20130603 & $23.060 \pm 0.050$ & $\mathrm{~T} 15 \mathrm{~b}$ \\
\hline SN2012fr & 101 & 20130221 & $15.696 \pm 0.015$ & Zhang et al. (2014) \\
\hline SN2012fr & 116 & 20130308 & $15.927 \pm 0.020$ & \\
\hline SN2012fr & 125 & 20130317 & $16.065 \pm 0.024$ & \\
\hline SN2012fr & 151 & 20130412 & $16.466 \pm 0.034$ & \\
\hline SN2012fr & 222 & 20130622 & $17.560 \pm 0.065$ & \\
\hline SN2012fr & 261 & 20130731 & $18.161 \pm 0.082$ & \\
\hline SN2012fr & 340 & 20131018 & $19.379 \pm 0.117$ & \\
\hline SN2012fr & 357 & 20131103 & $19.409 \pm 0.100$ & \\
\hline SN2012fr & 357 & 20131103 & $19.625 \pm 0.124$ & \\
\hline SN2012fr & 367 & 20131114 & $19.795 \pm 0.129$ & \\
\hline SN2012hr & 283 & 20131006 & $20.487 \pm 0.005$ & SN2003du \\
\hline SN2012hr & 368 & 20131230 & $21.749 \pm 0.007$ & \\
\hline SN2012ht & 437 & 20140313 & $22.714 \pm 0.100$ & $\mathrm{~T} 15 \mathrm{~b}$ \\
\hline SN2013aa & 137 & 20130710 & $15.768 \pm 0.000$ & SN1991T \\
\hline SN2013aa & 185 & 20130827 & $16.438 \pm 0.000$ & \\
\hline SN2013aa & 202 & 20130913 & $16.676 \pm 0.000$ & \\
\hline SN2013aa & 342 & 20140131 & $18.633 \pm 0.001$ & \\
\hline SN2013aa & 358 & 20140216 & $18.857 \pm 0.001$ & \\
\hline SN2013aa & 430 & 20140422 & $19.765 \pm 0.001$ & \\
\hline
\end{tabular}


Table B3 (cont'd)

\begin{tabular}{ccccc}
\hline \hline SN & $\begin{array}{c}\text { Phase } t \\
\text { (days) }\end{array}$ & Obs. Date & $\begin{array}{c}m_{B}(t) \\
(\mathrm{mag})\end{array}$ & Phot. Ref. \\
\hline SN2013cs & 320 & 20140322 & $20.909 \pm 0.068$ & SN2002fk \\
SN2013cs & 322 & 20140324 & $20.939 \pm 0.069$ & \\
SN2013cs & 322 & 20140324 & $21.475 \pm 0.100$ & \\
SN2013ct & 223 & 20131218 & $18.144 \pm 0.100$ & T15b \\
SN2013dl & 160 & 20141130 & $20.034 \pm 0.100$ & T15b \\
SN2013dl & 184 & 20141224 & $20.331 \pm 0.100$ & \\
SN2013dy & 333 & 20140626 & $20.716 \pm 0.033$ & Pan et al. (2015a) \\
SN2013dy & 419 & 20140920 & $22.064 \pm 0.045$ & \\
SN2013ef & 174 & 20141224 & $21.511 \pm 0.100$ & T15b \\
SN2013gy & 276 & 20140920 & $21.242 \pm 0.062$ & SN2007af \\
SN2014J & 231 & 20140920 & $16.956 \pm 0.119$ & Foley et al. (2014) \\
\hline
\end{tabular}

Note. - For SNe with poor late-time photometric coverage, the late light curve of a surrogate SN (denoted in boldface in the Photometry Reference column) is employed.

CfA: Riess et al. (1999); Jha et al. (2006); Hicken et al. (2009, 2012); LOSS:

Ganeshalingam et al. (2010); CSP: Contreras et al. (2010); Stritzinger et al. (2011) 
Table B4. Co line fluxes from spectra

\begin{tabular}{|c|c|c|c|c|c|}
\hline $\mathrm{SN}$ & Date & $\begin{array}{l}\text { Co Line Flux } \\
\left(\mathrm{erg} \mathrm{cm}^{2} \mathrm{~s}^{-1}\right)\end{array}$ & $\begin{array}{l}\text { B-Band Flux } \\
\left(\mathrm{erg} \mathrm{cm}^{2} \mathrm{~s}^{-1}\right)\end{array}$ & $\begin{array}{c}w_{\min } \\
(\AA)\end{array}$ & $\begin{array}{c}w_{\max } \\
(\AA)\end{array}$ \\
\hline SN1990N & 19901217 & $6.06 \times 10^{-16} \pm 3.39 \times 10^{-18}$ & $3.52 \times 10^{-15} \pm 1.95 \times 10^{-17}$ & 5710.5 & 6038 \\
\hline SN1990N & 19910112 & $5.99 \times 10^{-14} \pm 1.76 \times 10^{-17}$ & $3.98 \times 10^{-13} \pm 7.37 \times 10^{-17}$ & & \\
\hline SN1990N & 19910222 & $3.69 \times 10^{-14} \pm 8.73 \times 10^{-17}$ & $3.30 \times 10^{-13} \pm 5.29 \times 10^{-16}$ & & \\
\hline SN1990N & 19910322 & $9.78 \times 10^{-15} \pm 4.25 \times 10^{-17}$ & $1.28 \times 10^{-13} \pm 2.03 \times 10^{-16}$ & & \\
\hline SN1990N & 19910416 & $7.15 \times 10^{-15} \pm 5.64 \times 10^{-17}$ & $1.03 \times 10^{-13} \pm 2.24 \times 10^{-16}$ & & \\
\hline SN1990N & 19910608 & $1.89 \times 10^{-15} \pm 4.67 \times 10^{-17}$ & $5.34 \times 10^{-14} \pm 1.46 \times 10^{-16}$ & & \\
\hline SN1991M & 19910820 & $1.67 \times 10^{-16} \pm 5.39 \times 10^{-18}$ & $8.47 \times 10^{-16} \pm 1.29 \times 10^{-17}$ & 5770.8 & 6031.1 \\
\hline SN1991T & 19910819 & $4.99 \times 10^{-14} \pm 1.98 \times 10^{-16}$ & $1.28 \times 10^{-13} \pm 5.48 \times 10^{-16}$ & 5664.2 & 6121.0 \\
\hline SN1991T & 19911031 & $1.34 \times 10^{-15} \pm 2.23 \times 10^{-17}$ & $8.20 \times 10^{-15} \pm 4.80 \times 10^{-17}$ & & \\
\hline SN1991T & 1992 & $5.49 \times 10^{-14} \pm 8.12 \times 10^{-17}$ & $4.57 \times 10^{-13} \pm 2.40 \times 10^{-16}$ & & \\
\hline SN1991T & 19920313 & $1.18 \times 10^{-16} \pm 4.46 \times 10^{-18}$ & $1.09 \times 10^{-15} \pm 6.92 \times 10^{-18}$ & & \\
\hline SN1991T & 19920411 & $\times 10^{-17} \pm 2.51 \times 10^{-18}$ & $8.32 \times 10^{-16} \pm 3.12 \times 10^{-17}$ & & \\
\hline SN1993Z & 19940317 & $2.17 \times 10^{-16} \pm 3.14 \times 10^{-18}$ & $1.14 \times 10^{-15} \pm 1.00 \times 10^{-17}$ & 5757.8 & 6081.7 \\
\hline SN1993Z & 19940418 & $9.90 \times 10^{-17} \pm 2.47 \times 10^{-18}$ & $9.73 \times 10^{-16} \pm 5.87 \times 10^{-18}$ & & \\
\hline SN1994D & 19951124 & $4.69 \times 10^{-15} \pm 3.19 \times 10^{-16}$ & $4.88 \times 10^{-14} \pm 4.08 \times 10^{-16}$ & 5732.7 & 6070.7 \\
\hline SN1994ae & 19950422 & $6.74 \times 10^{-16} \pm 7.37 \times 10^{-18}$ & $3.77 \times 10^{-15} \pm 9.61 \times 10^{-18}$ & 5747.5 & 6027.4 \\
\hline SN1994ae & 19950501 & $5.38 \times 10^{-14} \pm 1.84 \times 10^{-16}$ & $4.04 \times 10^{-13} \pm 6.40 \times 10^{-16}$ & & \\
\hline SN1995D & 19951124 & $4.96 \times 10^{-15} \pm 3.49 \times 10^{-16}$ & $4.88 \times 10^{-14} \pm 4.08 \times 10^{-16}$ & 5677.7 & 6080.5 \\
\hline SN1995D & 19951202 & $1.96 \times 10^{-15} \pm 1.70 \times 10^{-16}$ & $2.67 \times 10^{-14} \pm 2.00 \times 10^{-16}$ & & \\
\hline SN1996X & 19970210 & $2.45 \times 10^{-15} \pm 3.74 \times 10^{-17}$ & $2.97 \times 10^{-14} \pm 1.09 \times 10^{-16}$ & 5681.8 & 6075.7 \\
\hline SN1998aq & 19981124 & $3.80 \times 10^{-14} \pm 1.20 \times 10^{-15}$ & $2.68 \times 10^{-13} \pm 2.37 \times 10^{-15}$ & & 6077.6 \\
\hline & 1998 & $10^{-14} \pm$ & $\times 10^{-13} \pm 1.01 \times 10^{-15}$ & & \\
\hline SN & 19 & $\times 10^{-14} \pm 7$ & $3.86 \times 10^{-13} \pm 1$ & & \\
\hline SN1998bu & 1998 & $13 \pm$ & $\times 10^{-13} \pm 1.95 \times$ & 5706.7 & 0 \\
\hline SN1998bu & 1998 & $1.39 \times 10^{-13} \pm 5.29 \times 10^{-16}$ & $6.45 \times 10^{-13} \pm 1.20 \times 10^{-15}$ & & \\
\hline SN1998bu & 19981213 & $8.76 \times 10^{-14} \pm 3.94 \times 10^{-16}$ & $5.13 \times 10^{-13} \pm 9.64 \times 10^{-16}$ & & \\
\hline SN1998bu & 19981222 & $8.04 \times 10^{-14} \pm 6.98 \times 10^{-16}$ & $4.24 \times 10^{-13} \pm 1.59 \times 10^{-15}$ & & \\
\hline SN1998bu & 19990110 & $4.58 \times 10^{-16} \pm 1.19 \times 10^{-18}$ & $3.71 \times 10^{-15} \pm 5.44 \times 10^{-18}$ & & \\
\hline SN1998bu & 19990117 & $5.47 \times 10^{-14} \pm 3.47 \times 10^{-16}$ & $2.95 \times 10^{-13} \pm 8.72 \times 10^{-16}$ & & \\
\hline SN1998bu & 19990223 & $2.08 \times 10^{-16} \pm 1.23 \times 10^{-18}$ & $1.72 \times 10^{-15} \pm 3.75 \times 10^{-18}$ & & \\
\hline SN1998bu & 19990413 & $6.52 \times 10^{-15} \pm 7.18 \times 10^{-17}$ & $6.01 \times 10^{-14} \pm 1.06 \times 10^{-16}$ & & \\
\hline SN1998bu & 19990424 & $1.42 \times 10^{-16} \pm 2.54 \times 10^{-18}$ & $1.04 \times 10^{-15} \pm 7.50 \times 10^{-18}$ & & \\
\hline SN1999aa & 19991109 & $1.80 \times 10^{-16} \pm 1.12 \times 10^{-18}$ & $8.69 \times 10^{-16} \pm 2.46 \times 10^{-18}$ & 5616.9 & 6197.1 \\
\hline $\mathrm{SN} 1$ & 1999 & $\times 10^{-17} \pm 8.1$ & $3.46 \times 10^{-16} \pm 2.46 \times 10^{-18}$ & & \\
\hline & 20 & $16 \pm 6$ & $10^{-16} \pm 2$ & & \\
\hline SN & & $15 \pm$ & $-14 \pm 3.97 \times$ & .4 & \\
\hline SN2002er & 2003 & $\times 10^{-15} \pm 8$ & $4.18 \times 10^{-14} \pm 2.10 \times 10^{-16}$ & 5709.1 & 6086.8 \\
\hline SN2002fk & 20030227 & $\times 10^{-16} \pm 6.66 \times 10^{-19}$ & $3.08 \times 10^{-15} \pm 2.47 \times 10^{-18}$ & 5739.0 & 6008.7 \\
\hline SN2003du & 20030823 & $1.36 \times 10^{-13} \pm 3.14 \times 10^{-16}$ & $5.08 \times 10^{-13} \pm 1.06 \times 10^{-15}$ & 5736.0 & 6027.0 \\
\hline SN2003du & 20030921 & $4.90 \times 10^{-14} \pm 9.36 \times 10^{-17}$ & $2.47 \times 10^{-13} \pm 4.95 \times 10^{-16}$ & & \\
\hline SN2003du & 20030922 & $4.24 \times 10^{-14} \pm 1.16 \times 10^{-16}$ & $2.12 \times 10^{-13} \pm 6.54 \times 10^{-16}$ & & \\
\hline SN2003du & 20030925 & $5.96 \times 10^{-14} \pm 1.36 \times 10^{-16}$ & $3.00 \times 10^{-13} \pm 7.96 \times 10^{-16}$ & & \\
\hline SN2003du & 20031201 & $1.39 \times 10^{-14} \pm 1.19 \times 10^{-16}$ & $1.05 \times 10^{-13} \pm 3.34 \times 10^{-16}$ & & \\
\hline SN2003du & 20031213 & $8.91 \times 10^{-15} \pm 1.06 \times 10^{-16}$ & $9.51 \times 10^{-14} \pm 2.63 \times 10^{-16}$ & & \\
\hline SN2003du & 20040202 & $3.34 \times 10^{-15} \pm 6.14 \times 10^{-17}$ & $6.68 \times 10^{-14} \pm 2.01 \times 10^{-16}$ & & \\
\hline & 200 & $-16 \pm 7$ & $1.24 \times 10^{-14} \pm 1$ & & \\
\hline $\mathrm{SN}$ & & 19 & 17 & 5762.4 & 6006.7 \\
\hline SN2003hv & 20031228 & $\times 10^{-13} \pm 1.28 \times 10^{-15}$ & $1.64 \times 10^{-12} \pm 2.68 \times 10^{-15}$ & 5687.6 & 6061.5 \\
\hline SN2003hv & 20040129 & $3.33 \times 10^{-13} \pm 1.31 \times 10^{-15}$ & $1.96 \times 10^{-12} \pm 3.52 \times 10^{-15}$ & & \\
\hline SN2003hv & 20040725 & $2.17 \times 10^{-15} \pm 1.56 \times 10^{-17}$ & $3.00 \times 10^{-14} \pm 3.21 \times 10^{-17}$ & & \\
\hline $004 \mathrm{bv}$ & 20041114 & $3.89 \times 10^{-16} \pm 1.05 \times 10^{-18}$ & $2.00 \times 10^{-15} \pm 1.87 \times 10^{-18}$ & 3 & 6057.6 \\
\hline SN2004eo & 20050516 & $1.46 \times 10^{-15} \pm 4.14 \times 10^{-18}$ & $1.22 \times 10^{-14} \pm 1.10 \times 10^{-17}$ & 5737.5 & 6034.0 \\
\hline SN2005cf & 20060427 & $4.44 \times 10^{-18} \pm 2.06 \times 10^{-19}$ & $5.34 \times 10^{-17} \pm 1.99 \times 10^{-19}$ & 5764.7 & 5992.4 \\
\hline SN2006X & 20060626 & $7.25 \times 10^{-16} \pm 1.12 \times 10^{-17}$ & $8.76 \times 10^{-16} \pm 1.13 \times 10^{-17}$ & 5670.0 & 6118.6 \\
\hline SN2006X & 20060721 & $3.12 \times 10^{-14} \pm 4.78 \times 10^{-16}$ & $5.35 \times 10^{-14} \pm 1.20 \times 10^{-15}$ & & \\
\hline SN2006X & 20061123 & $7.25 \times 10^{-17} \pm 5.51 \times 10^{-19}$ & $2.01 \times 10^{-16} \pm 7.62 \times 10^{-19}$ & & \\
\hline SN2006X & 20061223 & $3.70 \times 10^{-16} \pm 2.24 \times 10^{-18}$ & $5.19 \times 10^{-16} \pm 5.02 \times 10^{-17}$ & & \\
\hline
\end{tabular}


Table B4 (cont'd)

\begin{tabular}{|c|c|c|c|c|c|}
\hline SN & Date & $\begin{array}{l}\text { Co Line Flux } \\
\left(\mathrm{erg} \mathrm{cm}^{2} \mathrm{~s}^{-1}\right)\end{array}$ & $\begin{array}{l}\text { B-Band Flux } \\
\left(\mathrm{erg} \mathrm{cm}^{2} \mathrm{~s}^{-1}\right)\end{array}$ & $\begin{array}{c}w_{\min } \\
(\AA)\end{array}$ & $\begin{array}{c}w_{\max } \\
(\AA)\end{array}$ \\
\hline SN2006X & 070214 & $5.51 \times 10^{-17} \pm 1.42 \times 10^{-19}$ & $1.50 \times 10^{-16} \pm 4.34 \times 10^{-19}$ & & \\
\hline SN2007af & 20070620 & $2.96 \times 10^{-13} \pm 6.13 \times 10^{-16}$ & $1.09 \times 10^{-12} \pm 1.63 \times 10^{-15}$ & 5762.2 & 6027.6 \\
\hline SN2007af & 20070625 & $2.15 \times 10^{-13} \pm 5.45 \times 10^{-16}$ & $7.80 \times 10^{-13} \pm 2.19 \times 10^{-15}$ & & \\
\hline SN2007af & 20070707 & $3.06 \times 10^{-15} \pm 4.33 \times 10^{-18}$ & $1.36 \times 10^{-14} \pm 1.73 \times 10^{-17}$ & & \\
\hline SN2007af & 20070710 & $8.47 \times 10^{-14} \pm 4.97 \times 10^{-16}$ & $3.08 \times 10^{-13} \pm 1.39 \times 10^{-15}$ & & \\
\hline SN2007af & 20070715 & $8.25 \times 10^{-16} \pm 2.73 \times 10^{-18}$ & $4.00 \times 10^{-15} \pm 9.48 \times 10^{-18}$ & & \\
\hline SN2007af & 20070718 & $7.49 \times 10^{-14} \pm 6.87 \times 10^{-16}$ & $3.47 \times 10^{-13} \pm 3.23 \times 10^{-15}$ & & \\
\hline SN2007af & 20070807 & $2.50 \times 10^{-16} \pm 3.72 \times 10^{-18}$ & $1.67 \times 10^{-15} \pm 1.04 \times 10^{-17}$ & & \\
\hline SN2007af & 20070821 & $3.57 \times 10^{-16} \pm 4.16 \times 10^{-18}$ & $2.15 \times 10^{-15} \pm 1.93 \times 10^{-17}$ & & \\
\hline SN2007af & 20080111 & $2.19 \times 10^{-15} \pm 4.58 \times 10^{-17}$ & $3.08 \times 10^{-14} \pm 8.19 \times 10^{-17}$ & & \\
\hline SN2007gi & 20080115 & $1.20 \times 10^{-15} \pm 3.12 \times 10^{-18}$ & $5.08 \times 10^{-15} \pm 8.44 \times 10^{-18}$ & 5706.8 & 6146.6 \\
\hline SN2007le & 080827 & $80 \times 10^{-17} \pm 4.41 \times 10^{-19}$ & $2.02 \times 10^{-16} \pm 5.68 \times 10^{-19}$ & 5721.8 & 6087.5 \\
\hline SN2007sr & 20080623 & $4.42 \times 10^{-14} \pm 5.84 \times 10^{-17}$ & $3.05 \times 10^{-13} \pm 4.04 \times 10^{-16}$ & 5765.7 & 6059.7 \\
\hline SN2008Q & 20080828 & $9.20 \times 10^{-17} \pm 2.08 \times 10^{-19}$ & $6.25 \times 10^{-16} \pm 5.91 \times 10^{-19}$ & 5691.4 & 6089.5 \\
\hline SN2009ig & 20101017 & $1.50 \times 10^{-15} \pm 6.87 \times 10^{-18}$ & $1.23 \times 10^{-14} \pm 1.85 \times 10^{-17}$ & 5496.8 & 6156.7 \\
\hline SN2009le & 20101016 & $5.18 \times 10^{-16} \pm 9.02 \times 10^{-18}$ & $5.27 \times 10^{-15} \pm 2.33 \times 10^{-17}$ & 5587.7 & 6195.9 \\
\hline SN2010ev & 20101231 & $1.23 \times 10^{-14} \pm 2.27 \times 10^{-17}$ & $5.05 \times 10^{-14} \pm 5.09 \times 10^{-17}$ & 56 & 6152.9 \\
\hline SN2010ev & 2011 & $\times 10^{-15} \pm 8.29 \times 10^{-18}$ & $2.04 \times 10^{-14} \pm 3.81 \times 10^{-17}$ & & \\
\hline SN2010gp & & $2.82 \times 10^{-16} \pm 4$ & $3 \times 10^{-15} \pm$ & 6 & 6 \\
\hline SN2010hg & & $\times 10^{-15} \pm 2.08 \times 10^{-17}$ & $\times 10^{-14} \pm 7$ & & \\
\hline SN2010kg & 20110927 & $5 \times 10^{-16} \pm 9.49 \times 10^{-18}$ & $5.57 \times 10^{-15} \pm 3.43 \times 10^{-17}$ & & 6165.6 \\
\hline SN2011ae & 120101 & $2.70 \times 10^{-15} \pm 2.21 \times 10^{-17}$ & $3.27 \times 10^{-14} \pm 7.28 \times 10^{-17}$ & 5684.3 & 6087.6 \\
\hline SN2011at & 27 & $.93 \times 10^{-15} \pm 8.90 \times 10^{-18}$ & $1.32 \times 10^{-14} \pm 2.58 \times 10^{-17}$ & 5618.1 & 6118.7 \\
\hline SN2011by & 02 & $1.85 \times 10^{-15} \pm 4.98 \times 10^{-18}$ & $1.53 \times 10^{-14} \pm 1.68 \times 10^{-17}$ & 5723.6 & 6039.5 \\
\hline SN2011by & 20120315 & $5.84 \times 10^{-17} \pm 4.32 \times 10^{-19}$ & $8.72 \times 10^{-16} \pm 1.26 \times 10^{-18}$ & & \\
\hline SN2011ek & 20121011 & $1.73 \times 10^{-16} \pm 4.75 \times 10^{-18}$ & $1.12 \times 10^{-15} \pm 9.34 \times 10^{-18}$ & 5544.0 & 6130.4 \\
\hline SN2011fe & 20111123 & $7.06 \times 10^{-12} \pm 1.78 \times 10^{-15}$ & $1.76 \times 10^{-11} \pm 5.31 \times 10^{-15}$ & 5738.3 & 6027.2 \\
\hline SN2011fe & 20120102 & $1.38 \times 10^{-12} \pm 2.86 \times 10^{-16}$ & $5.87 \times 10^{-12} \pm 1.76 \times 10^{-15}$ & & \\
\hline SN2011fe & 20120 & $2.14 \times 10^{-13} \pm 5.88 \times 10^{-17}$ & $1.81 \times 10^{-12} \pm 5.28 \times 10^{-16}$ & & \\
\hline SN2011fe & & $\times 10^{-14} \pm 2.34 \times 10^{-17}$ & $8.94 \times 10^{-13} \pm 2.50 \times 10^{-16}$ & & \\
\hline & & $10^{-17}$ & $\times 10^{-13} \pm 2$ & & \\
\hline SN & & -17 & $-13 \pm$ & & \\
\hline im & & $\times 10^{-16} \pm 7.58 \times 10^{-18}$ & $2.36 \times 10^{-15} \pm 1.93 \times 10^{-17}$ & & 6119.4 \\
\hline 11 iv & & $\times 10^{-15} \pm 4.00 \times 10^{-17}$ & $3.13 \times 10^{-14} \pm 8.86 \times 10^{-17}$ & 5693.8 & 6089.2 \\
\hline SN2012cg & 30507 & $3.98 \times 10^{-15} \pm 5.08 \times 10^{-17}$ & $5.30 \times 10^{-14} \pm 6.44 \times 10^{-17}$ & 5698.9 & 6113.3 \\
\hline SN2012cg & 130513 & $4.30 \times 10^{-15} \pm 3.59 \times 10^{-17}$ & $6.51 \times 10^{-14} \pm 1.22 \times 10^{-16}$ & & \\
\hline SN2012cu & 130603 & $6.26 \times 10^{-16} \pm 7.42 \times 10^{-18}$ & $3.62 \times 10^{-15} \pm 2.06 \times 10^{-17}$ & 5692.6 & 6096.8 \\
\hline SN2012fr & 20130221 & $6.02 \times 10^{-13} \pm 6.43 \times 10^{-16}$ & $1.97 \times 10^{-12} \pm 2.79 \times 10^{-15}$ & 5759.2 & 6061.5 \\
\hline SN2012fr & 20130308 & $3.30 \times 10^{-13} \pm 4.81 \times 10^{-16}$ & $1.28 \times 10^{-12} \pm 2.34 \times 10^{-15}$ & & \\
\hline SN2012fr & 20130317 & $3.52 \times 10^{-13} \pm 4.66 \times 10^{-16}$ & $1.42 \times 10^{-12} \pm 1.48 \times 10^{-15}$ & & \\
\hline SN2012fr & & $2.22 \times 10^{-13} \pm 1.51 \times 10^{-15}$ & $1.49 \times 10^{-12} \pm 4.85 \times 10^{-15}$ & & \\
\hline SN2012fr & & $\times 10^{-14} \pm 3$ & $3.57 \times 10^{-13} \pm 1.62 \times 10^{-15}$ & & \\
\hline & & -16 & $-13 \pm$ & & \\
\hline $\mathrm{SN}$ & & 16 & 6 & & \\
\hline SN2012fr & & $47 \times 10^{-15} \pm 2.94 \times 10^{-18}$ & $1.19 \times 10^{-13} \pm 1.98 \times 10^{-17}$ & & \\
\hline $12 \mathrm{fr}$ & 31114 & $1.25 \times 10^{-15} \pm 1.05 \times 10^{-16}$ & $4.16 \times 10^{-14} \pm 3.91 \times 10^{-16}$ & & \\
\hline SN2012hr & 20131006 & $3.61 \times 10^{-17} \pm 2.28 \times 10^{-19}$ & $5.34 \times 10^{-16} \pm 7.39 \times 10^{-19}$ & 5775.3 & 6041 \\
\hline SN2012hr & 20131230 & $2.53 \times 10^{-16} \pm 2.76 \times 10^{-17}$ & $7.26 \times 10^{-15} \pm 1.39 \times 10^{-16}$ & & \\
\hline SN2013aa & 20130710 & $3.75 \times 10^{-13} \pm 4.69 \times 10^{-16}$ & $1.60 \times 10^{-12} \pm 1.07 \times 10^{-15}$ & 5720.3 & 6055.9 \\
\hline SN2013aa & 20130827 & $5.88 \times 10^{-14} \pm 1.19 \times 10^{-16}$ & $4.14 \times 10^{-13} \pm 5.40 \times 10^{-16}$ & & \\
\hline SN2013aa & 20130913 & $6.54 \times 10^{-14} \pm 4.35 \times 10^{-16}$ & $5.13 \times 10^{-13} \pm 1.62 \times 10^{-15}$ & & \\
\hline SN2013aa & 20140131 & $1.76 \times 10^{-15} \pm 1.48 \times 10^{-16}$ & $5.26 \times 10^{-14} \pm 3.92 \times 10^{-16}$ & & \\
\hline SN2013aa & 20140216 & $2.10 \times 10^{-15} \pm 2.73 \times 10^{-17}$ & $3.78 \times 10^{-14} \pm 4.23 \times 10^{-17}$ & & \\
\hline SN2013aa & 20140422 & $8.32 \times 10^{-16} \pm 2.73 \times 10^{-17}$ & $1.61 \times 10^{-14} \pm 3.68 \times 10^{-17}$ & & \\
\hline SN2013cs & 20140322 & $3.28 \times 10^{-16} \pm 9.26 \times 10^{-17}$ & $7.49 \times 10^{-15} \pm 3.94 \times 10^{-16}$ & 5735.8 & 6061 \\
\hline SN2013cs & 20140324 & $9.99 \times 10^{-16} \pm 1.80 \times 10^{-17}$ & $1.56 \times 10^{-14} \pm 2.24 \times 10^{-17}$ & & \\
\hline SN2013ct & 20131218 & $3.71 \times 10^{-14} \pm 8.54 \times 10^{-17}$ & $3.35 \times 10^{-13} \pm 2.32 \times 10^{-16}$ & 5753.1 & 6030.3 \\
\hline
\end{tabular}


Childress et al.

Table B4 (cont'd)

\begin{tabular}{cccccc}
\hline \hline SN & Date & $\begin{array}{c}\text { Co Line Flux } \\
\left(\mathrm{erg} \mathrm{cm}^{2} \mathrm{~s}^{-1}\right)\end{array}$ & $\begin{array}{c}\text { B-Band Flux } \\
\left(\mathrm{erg} \mathrm{cm}^{2} \mathrm{~s}^{-1}\right)\end{array}$ & $\begin{array}{c}w_{\min } \\
(\AA)\end{array}$ & $\begin{array}{c}w_{\max } \\
(\AA)\end{array}$ \\
\hline SN2013dl & 20141130 & $1.17 \times 10^{-14} \pm 6.49 \times 10^{-17}$ & $5.87 \times 10^{-14} \pm 1.04 \times 10^{-16}$ & 5763.5 & 6061.3 \\
SN2013dl & 20141224 & $7.29 \times 10^{-15} \pm 6.95 \times 10^{-17}$ & $4.47 \times 10^{-14} \pm 8.46 \times 10^{-17}$ & & \\
SN2013dy & 20140626 & $5.54 \times 10^{-15} \pm 3.61 \times 10^{-17}$ & $6.29 \times 10^{-14} \pm 3.65 \times 10^{-15}$ & 5636.9 & 6168.7 \\
SN2013dy & 20140920 & $1.45 \times 10^{-14} \pm 1.34 \times 10^{-16}$ & $1.00 \times 10^{-13} \pm 6.47 \times 10^{-15}$ & & \\
SN2013ef & 20141224 & $3.28 \times 10^{-15} \pm 3.58 \times 10^{-17}$ & $1.51 \times 10^{-14} \pm 5.48 \times 10^{-17}$ & 5733.8 & 6053.0 \\
SN2013gy & 20140920 & $7.63 \times 10^{-15} \pm 5.97 \times 10^{-17}$ & $5.72 \times 10^{-14} \pm 1.82 \times 10^{-15}$ & 5726.8 & 6064.7 \\
SN2014J & 20140920 & $1.65 \times 10^{-12} \pm 7.92 \times 10^{-15}$ & $5.19 \times 10^{-12} \pm 9.56 \times 10^{-15}$ & 5737.8 & 6069.5 \\
\hline
\end{tabular}

